Supporting Information

\title{
A Double-Walled Knotted Cage for Guest-Adaptive
}

\section{Molecular Recognition}

Yukari Tamura $^{\dagger}$, Hiroki Takezawa ${ }^{*}$, , and Makoto Fujita ${ }^{*}, \dagger$

†Department of Applied Chemistry, School of Engineering, The University of Tokyo, 7-3-1 Hongo, Bunkyo-ku, Tokyo 113-8656, Japan. ${ }^{*}$ Division of Advanced Molecular Science, Institute for Molecular Science (IMS), 5-1 Higashiyama, Myodaiji, Okazaki, Aichi 444-8787, Japan

E-mail:

\section{Contents}

$\begin{array}{lr}\text { 1. Materials and instrumentations } & \text { S2 }\end{array}$

$\begin{array}{ll}\text { 2. Synthesis and physical properties of ligand } 2 & \text { S3 }\end{array}$

$\begin{array}{ll}\text { 3. Synthesis and physical properties of cage } \mathbf{1} & \text { S8 }\end{array}$

$\begin{array}{lr}\text { 4. Inclusion experiments } & \text { S17 }\end{array}$

5. X-ray crystallographic analyses of cage $\mathbf{1}, \mathbf{1} \cdot \mathbf{C C l}_{\mathbf{4}}$, and $\mathbf{S 3} \mathrm{S} 31$

$\begin{array}{ll}\text { 6. Sequential guest binding } & \text { S35 }\end{array}$ 


\section{Materials and instrumentations}

${ }^{1} \mathrm{H}$ and ${ }^{13} \mathrm{C}$ NMR spectra were recorded on a Bruker AVANCE III or a Bruker AVANCE 500 equipped with CP-TCI cryoprobe (500 MHz for ${ }^{1} \mathrm{H}$ NMR and $125 \mathrm{MHz}$ for ${ }^{13} \mathrm{C}$ NMR) at $300 \mathrm{~K}$ unless otherwise stated. TMS $\left(\mathrm{CDCl}_{3}\right.$ solution) in a capillary served as an internal standard for ${ }^{1} \mathrm{H}$ NMR $(\delta=0 \mathrm{ppm})$. GCMS data were obtained on an Agilent 5977B inert Mass selective Detector equipped with a 7820A Network GC system and an EI source. ESI-MS data were recorded on a Bruker maXis. The MALDI-TOF mass spectra were obtained using a Bruker Daltonics Autoflex Speed spectrometer with dithranol as the matrix. IR measurements were carried out using a DIGILAB Scimitar FTS-2000 instrument. Melting points were determined on a Stanford Research Systems OptiMelt. Elemental analyses were performed at the Elemental Analysis Center (School of Science, The University of Tokyo). Single crystal X-ray diffraction data were collected on a BRUKER APEX-II CCD rotating anode diffractometer equipped with focusing mirrors with $\operatorname{MoK}_{\alpha}(\lambda=0.71073 \AA)$ radiation under cryogenic conditions, which are controlled with a cryostat system equipped with an $\mathrm{N}_{2}$ generator (Japan Thermal Eng. Co., Ltd.) or a Synergy-S diffractometer (Rigaku Oxford Diffraction), which is equipped with a micro-focus $\mathrm{Cu} K_{\alpha}$ radiation source $(\lambda=1.5418 \AA$ ), a highsensitive CCD detector, and a low temperature system using cold nitrogen stream (100 K). Solvents and reagents were purchased from TCI Co., Ltd., Wako Pure Chemical Industries Ltd., Kanto Chemical Co., Inc., and Sigma-Aldrich Co. and used without any further purification. 


\section{Synthesis and physical properties of ligand 2}

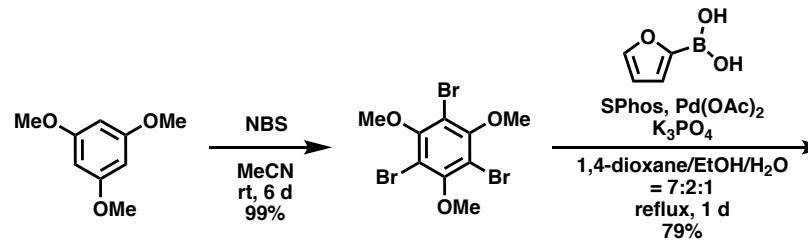

S1

S2

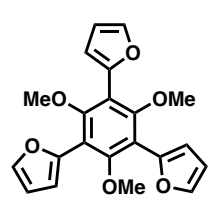

S3

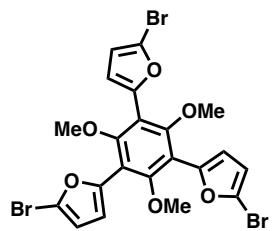

S4
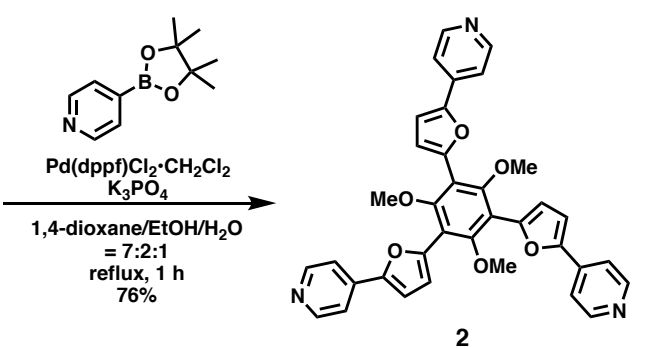

1,3,5-Tribromo-2,4,6-trimethoxybenzene (S2)

A mixture of 1,3,5-trimethoxybenzene (S1, $5.00 \mathrm{~g}, 29.7 \mathrm{mmol})$ and $\mathrm{N}$-bromosuccinimide $(21.0 \mathrm{~g}, 118$ $\mathrm{mmol})$ in $\mathrm{CH}_{3} \mathrm{CN}(50 \mathrm{~mL})$ was stirred at room temperature for $6 \mathrm{~d}$. The solvent was removed under reduced pressure, and EtOAc was added. The solution was washed with $\mathrm{H}_{2} \mathrm{O}, \mathrm{Na}_{2} \mathrm{~S}_{2} \mathrm{O}_{4}$ aq. and $\mathrm{NaHCO}_{3}$ aq. The organic layer was dried over anhydrous $\mathrm{Na}_{2} \mathrm{SO}_{4}$ and filtered. The solvent was removed under reduced pressure to give 1,3,5-tribromo-2,4,6-trimethoxybenzene (S2) quantitatively as a white powder (11.9 g, $29.3 \mathrm{mmol}, 99 \%)^{\mathrm{S} 1}$

Physical data of S2: white powder. ${ }^{1} \mathrm{H}$ NMR $\left(\mathrm{CDCl}_{3}, 500 \mathrm{MHz}\right): \delta 3.89\left(\mathrm{~s}, 9 \mathrm{H}, \mathrm{CH}_{3}\right),{ }^{13} \mathrm{C} \mathrm{NMR}\left(\mathrm{CDCl}_{3}\right.$, $125 \mathrm{MHz}): \delta 155.0(\mathrm{C}), 110.1(\mathrm{CBr}), 60.7\left(\mathrm{CH}_{3}\right)$. GC/MS (EI) $\mathrm{m} / \mathrm{z}: 405.8[\mathrm{M}]^{+}$.

\section{1,3,5-Tris(2-furyl)-2,4,6-trimethoxybenzene (S3)}

A mixture of 1,3,5-tribromo-2,4,6-trimethoxybenzene ( $\mathbf{S 2}, 5.01 \mathrm{~g}, 12.3 \mathrm{mmol}$ ), 2-furylboronic acid (4.97 g, $44.5 \mathrm{mmol}), \mathrm{Pd}(\mathrm{OAc})_{2}(280 \mathrm{mg}, 1.23 \mathrm{mmol})$, SPhos (1.01 g, $\left.2.47 \mathrm{mmol}\right)$ and $\mathrm{K}_{3} \mathrm{PO}_{4}(13.0 \mathrm{~g}, 61.7 \mathrm{mmol})$ in a mixed solvent of 1,4-dioxane $(28 \mathrm{~mL})$, ethanol $(8 \mathrm{~mL})$ and $\mathrm{H}_{2} \mathrm{O}(4 \mathrm{~mL})$ was refluxed under $\mathrm{Ar}$ atmosphere for $19 \mathrm{~h} . \mathrm{CHCl}_{3}(80 \mathrm{~mL})$ was added to the mixture and the organic layer was collected and washed with $\mathrm{H}_{2} \mathrm{O}(100 \mathrm{~mL} \times 2)$ and brine $(100 \mathrm{~mL} \times 2)$. The organic layer was dried over anhydrous $\mathrm{Na}_{2} \mathrm{SO}_{4}$ and filtered. The solvent was removed under reduced pressure. The crude product was purified by column chromatography on silica gel ( $n$-hexane/EtOAc $=98: 2)$ to provide 1,3,5-tris(2-furyl)-2,4,6trimethoxybenzene (S3) as a white crystalline powder $(3.56 \mathrm{~g}, 9.72 \mathrm{mmol}, 79 \%){ }^{\mathrm{S} 2}$ 
Physical data of S3: white crystalline powder. ${ }^{1} \mathrm{H} \mathrm{NMR}\left(\mathrm{CDCl}_{3}, 500 \mathrm{MHz}\right): \delta 7.57$ (t, $J=1.0 \mathrm{~Hz}, 3 \mathrm{H}$, $\operatorname{Ar} H), 6.64(\mathrm{dd}, J=0.8,3.3 \mathrm{~Hz}, 3 \mathrm{H}, \mathrm{Ar} H), 6.53(\mathrm{dd}, J=1.8,3.3 \mathrm{~Hz}, 3 \mathrm{H}, \mathrm{Ar} H), 3.39$ (s, $\left.9 \mathrm{H}, \mathrm{CH}_{3}\right) ;{ }^{13} \mathrm{C} \mathrm{NMR}$ $\left(\mathrm{CDCl}_{3}, 125 \mathrm{MHz}\right): \delta 158.5(\mathrm{C}), 146.2(\mathrm{C}), 142.3(\mathrm{CH}), 116.1(\mathrm{C}), 111.1(\mathrm{CH}), 111.0(\mathrm{CH}), 61.2\left(\mathrm{CH}_{3}\right)$. GC/MS (EI) $m / z: 366.1[\mathrm{M}]^{+}$.

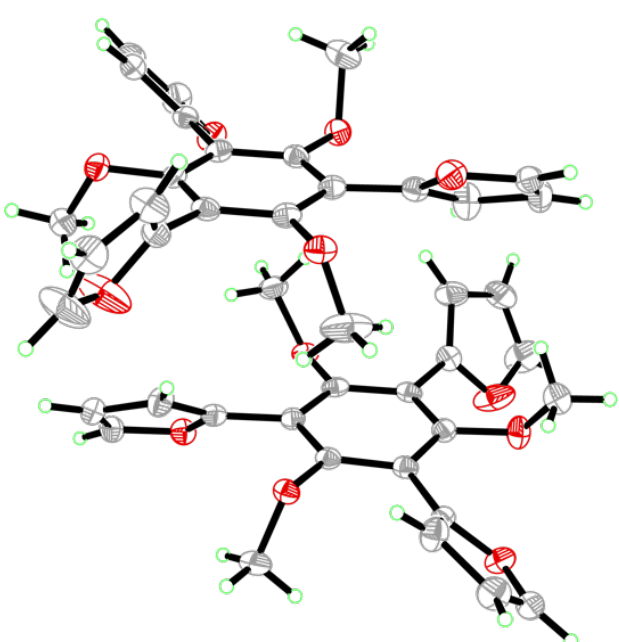

Figure S1. ORTEP drawing (50\% probability ellipsoids) of the refinement structure of S3 (CCDC reference number: 1984215).

\section{1,3,5-Tris(5-bromofuran-2-yl)-2,4,6-trimethoxybenzene (S4)}

A mixture of 1,3,5-tris(2-furyl)-2,4,6-trimethoxybenzene (S3, $1.00 \mathrm{~g}, 2.73 \mathrm{mmol})$ and $\mathrm{N}$ bromosuccinimide (1.61 g, $9.04 \mathrm{mmol})$ in $N, N$-dimethylformamide $(10 \mathrm{~mL})$ was stirred at room temperature for $1 \mathrm{~h} . \mathrm{Et}_{2} \mathrm{O}(50 \mathrm{~mL})$ and $\mathrm{H}_{2} \mathrm{O}(150 \mathrm{~mL})$ were added to the mixture and the organic layer was collected. The organic layer was washed with $\mathrm{H}_{2} \mathrm{O}, \mathrm{Na}_{2} \mathrm{~S}_{2} \mathrm{O}_{4}$ aq. and $\mathrm{NaHCO}_{3}$ aq. then dried over anhydrous $\mathrm{Na}_{2} \mathrm{SO}_{4}$ and filtered. The solvent was removed under reduced pressure. The crude product was purified by column chromatography on silica gel $(n$-hexane/EtOAc $=9: 1)$ to provide 1,3,5-tris (5-bromofuran-2-yl)2,4,6-trimethoxybenzene (S4) as a white powder (1.55 g, $2.57 \mathrm{mmol}, 94 \%)$.

Physical data of S4: white powder. ${ }^{1} \mathrm{H}$ NMR ( $\left.\mathrm{CDCl}_{3}, 500 \mathrm{MHz}\right): \delta 6.60$ (d, $\left.J=3.0 \mathrm{~Hz}, 3 \mathrm{H}, \mathrm{ArH}\right), 6.44$ (d, $J=3.0 \mathrm{~Hz}, 3 \mathrm{H}, \mathrm{Ar} H), 3.48$ (s, 9H, $\left.\mathrm{CH}_{3}\right) ;{ }^{13} \mathrm{C} \mathrm{NMR}\left(\mathrm{CDCl}_{3}, 125 \mathrm{MHz}\right): \delta 158.8(C), 148.1(C), 121.8(C)$, $115.6(\mathrm{C}), 114.1(\mathrm{CH}), 112.9(\mathrm{CH}), 61.7\left(\mathrm{CH}_{3}\right)$. IR (ATR, $\left.\mathrm{cm}^{-1}\right)$ : 2359, 2344, 2335, 2327, 2321, 1080, 1016, 956, 931, 776, 719, 666. m.p.: 125-126 ${ }^{\circ} \mathrm{C}$. MALDI-TOF-MS (ESI): calcd. for [M] $]^{+}: 599.8$, found 599.6. Elemental analysis (\%): calcd. for $\mathrm{C}_{21} \mathrm{H}_{15} \mathrm{Br}_{3} \mathrm{O}_{6}: \mathrm{C} 41.83, \mathrm{H}$ 2.51, N: 0.00; found: C 41.94, H 2.70, N 0.00 . 


\section{1,3,5-Tris(5-(prydine-4-yl)furan-2-yl)-2,4,6-trimethoxybenzene (2)}

A mixture of 1,3,5-tris(5-bromofuran-2-yl)-2,4,6-trimethoxybenzene (S4, $511 \mathrm{mg}, 0.847 \mathrm{mmol}$ ), 4pyridylboronic acid pinacol ester $(571 \mathrm{mg}, 2.78 \mathrm{mmol}), \mathrm{Pd}(\mathrm{dppf}) \mathrm{Cl}_{2} \cdot \mathrm{CH}_{2} \mathrm{Cl}_{2}(206 \mathrm{mg}, 0.252 \mathrm{mmol})$ and $\mathrm{K}_{3} \mathrm{PO}_{4}(891 \mathrm{mg}, 4.20 \mathrm{mmol})$ in a mixed solvent of 1,4-dioxane $(21 \mathrm{~mL})$, ethanol $(6 \mathrm{~mL})$ and $\mathrm{H}_{2} \mathrm{O}(3 \mathrm{~mL})$ was refluxed under Ar atmosphere for $1 \mathrm{~h}$. EtOAc $(80 \mathrm{~mL})$ was added to the mixture and filtered. The organic layer was washed with $\mathrm{H}_{2} \mathrm{O}(100 \mathrm{~mL} \times 3)$ and extracted with $50 \mathrm{mM} \mathrm{HCl} \mathrm{aq}(60 \mathrm{~mL} \times 2)$. The aqueous layer was neutralized with $\mathrm{NaOH}$ aq. and extracted with EtOAc. The organic layer was dried over anhydrous $\mathrm{Na}_{2} \mathrm{SO}_{4}$ and filtered. The solvent was removed under reduced pressure. The crude product was purified by recrystallization from EtOAc and $n$-hexane to provide 1,3,5-tris(5-(pyridine-4-yl)furan-2-yl)2,4,6-trimethoxybenzene (2) as an off-white powder (385 mg, $0.644 \mathrm{mmol}, 76 \%$ ).

Physical data of 2: off-white powder. ${ }^{1} \mathrm{H}$ NMR ( $\left.\mathrm{CDCl}_{3}, 500 \mathrm{MHz}\right): \delta 8.61\left(\mathrm{~d}, J=5.5 \mathrm{~Hz}, 6 \mathrm{H}, \mathrm{Py} H_{\alpha}\right), 7.58$ (d, $\left.J=5.5 \mathrm{~Hz}, 6 \mathrm{H}, \mathrm{Py} H_{\beta}\right), 7.06$ (d, $\left.J=3.5 \mathrm{~Hz}, 3 \mathrm{H}, \mathrm{Ar} H\right), 6.86$ (d, $\left.J=3.5 \mathrm{~Hz}, 3 \mathrm{H}, \mathrm{Ar} H\right), 3.57$ (s, 9H, CH$)_{3}$ )

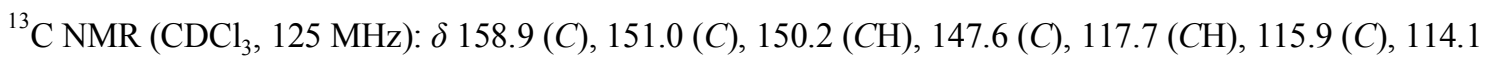
$(\mathrm{CH}), 110.4(\mathrm{CH}), 61.7\left(\mathrm{CH}_{3}\right)$. IR (ATR, $\left.\mathrm{cm}^{-1}\right): 2973,2935$, 2363, 1592, 1455, 1080, 730, 712. m.p.: 168 $169^{\circ} \mathrm{C}$. HR-MS (ESI): calcd for $[\mathrm{M}+\mathrm{H}]^{+}: 598.1973$, found: 598.1998 .
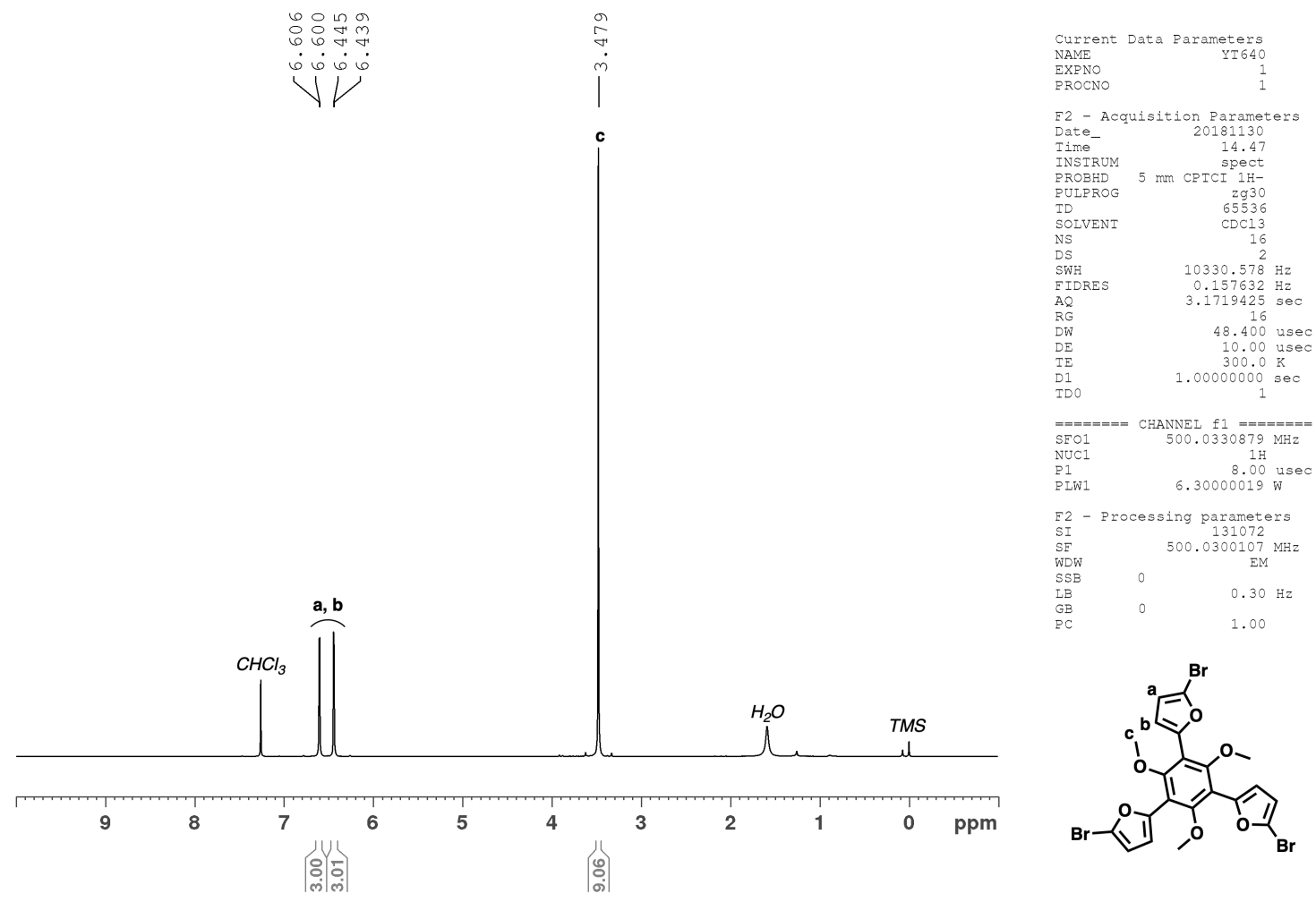

Figure S2. ${ }^{1} \mathrm{H}$ NMR spectrum $\left(500 \mathrm{MHz}, 300 \mathrm{~K}, \mathrm{CDCl}_{3}\right)$ of $\mathbf{S 4}$. 

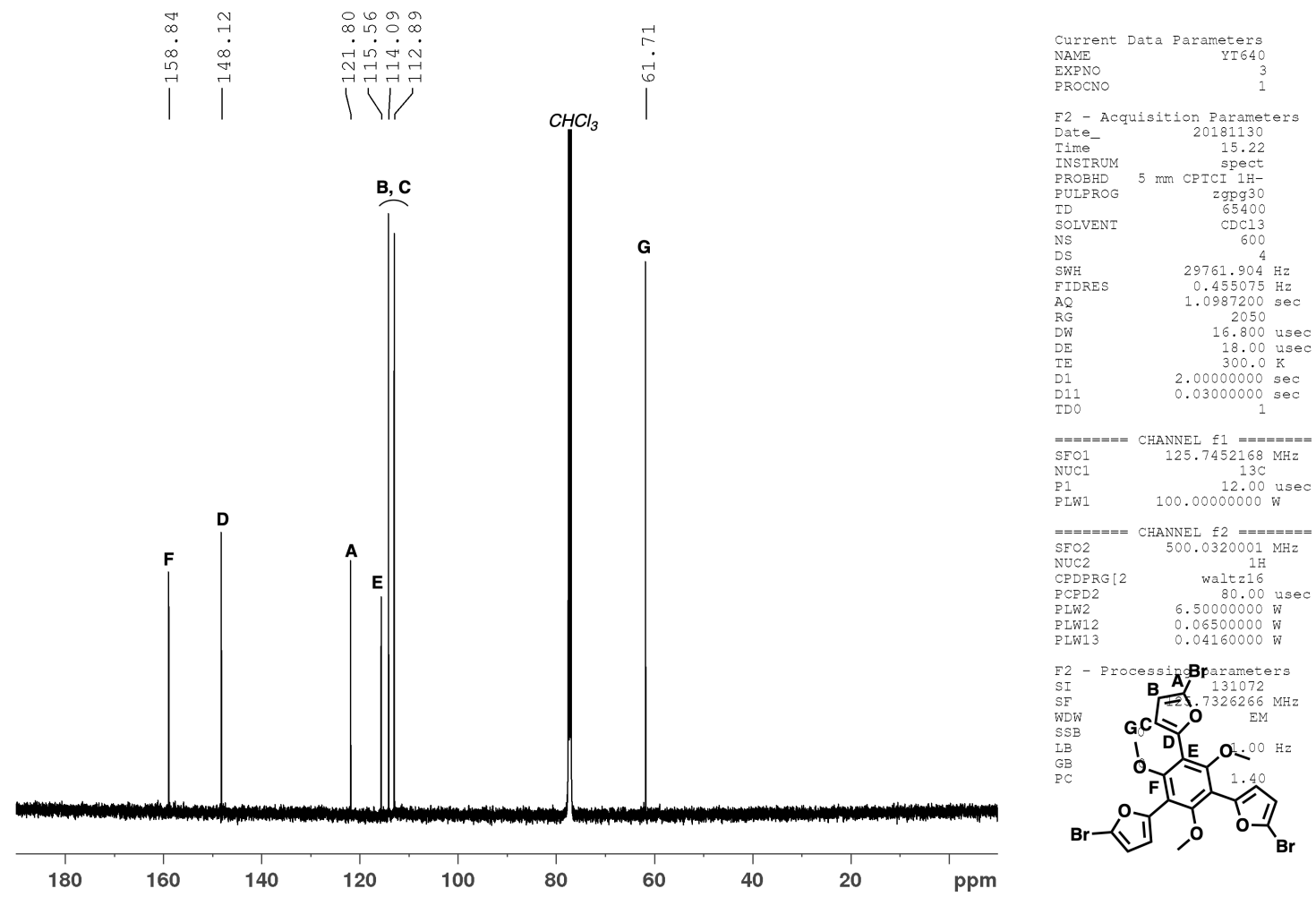

Figure S3. ${ }^{13} \mathrm{C}$ NMR spectrum $\left(125 \mathrm{MHz}, 300 \mathrm{~K}, \mathrm{CDCl}_{3}\right)$ of $\mathbf{S 4}$.
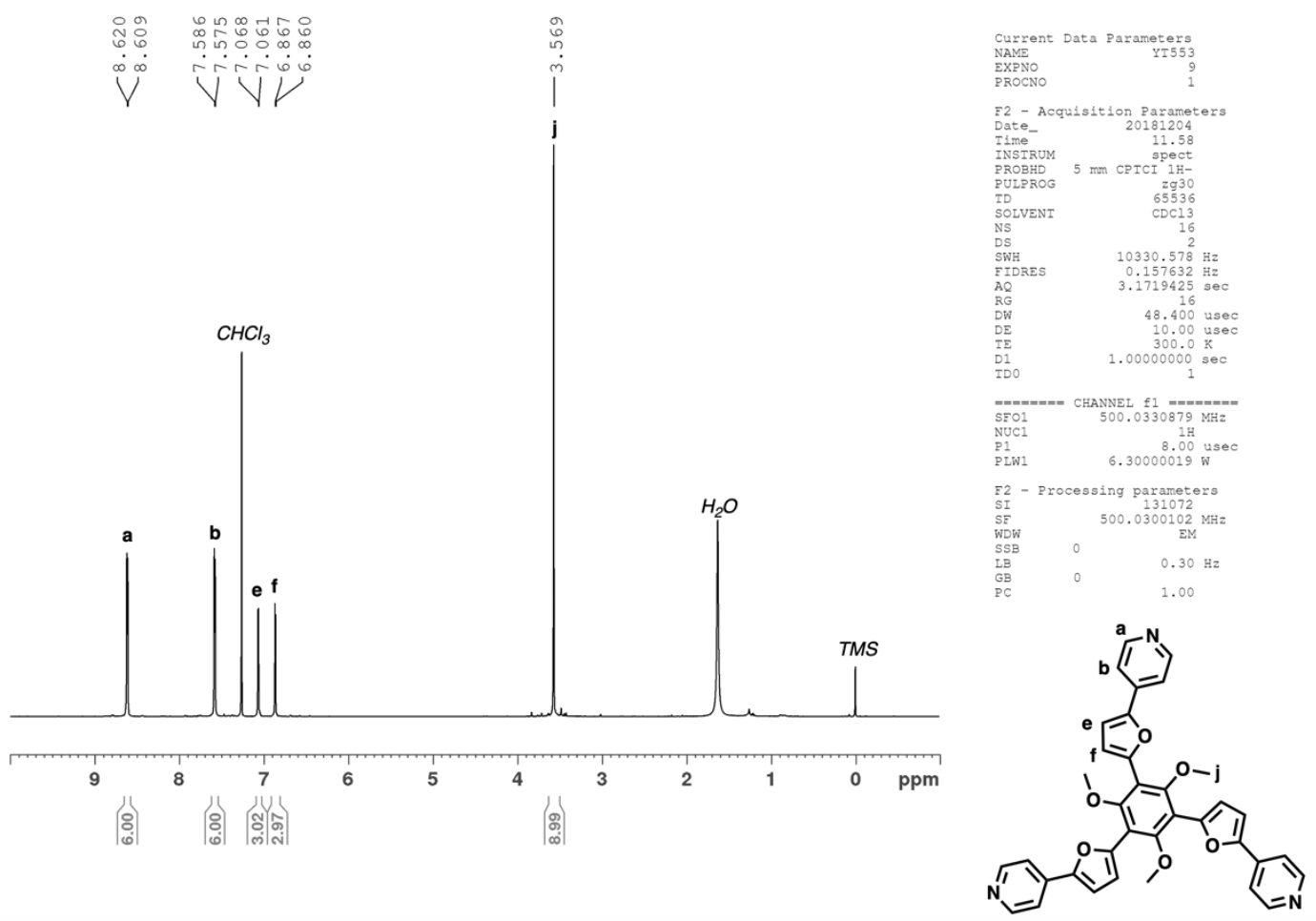

Figure S4. ${ }^{1} \mathrm{H}$ NMR spectrum $\left(500 \mathrm{MHz}, 300 \mathrm{~K}, \mathrm{CDCl}_{3}\right)$ of 2. 


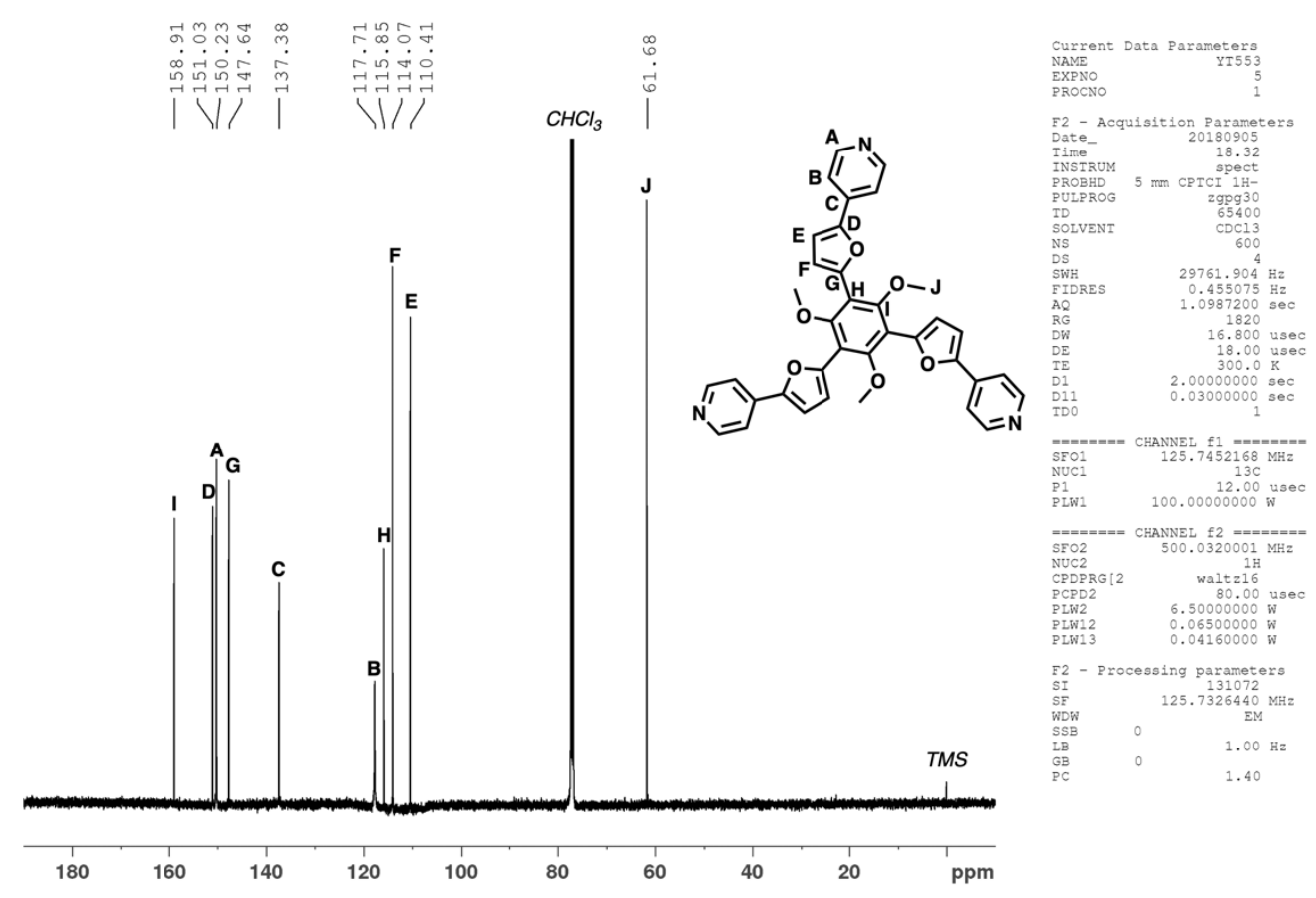

Figure S5. ${ }^{13} \mathrm{C}$ NMR spectrum $\left(125 \mathrm{MHz}, 300 \mathrm{~K}, \mathrm{CDCl}_{3}\right)$ of 2.

ST 


\section{Synthesis and physical properties of cage 1}

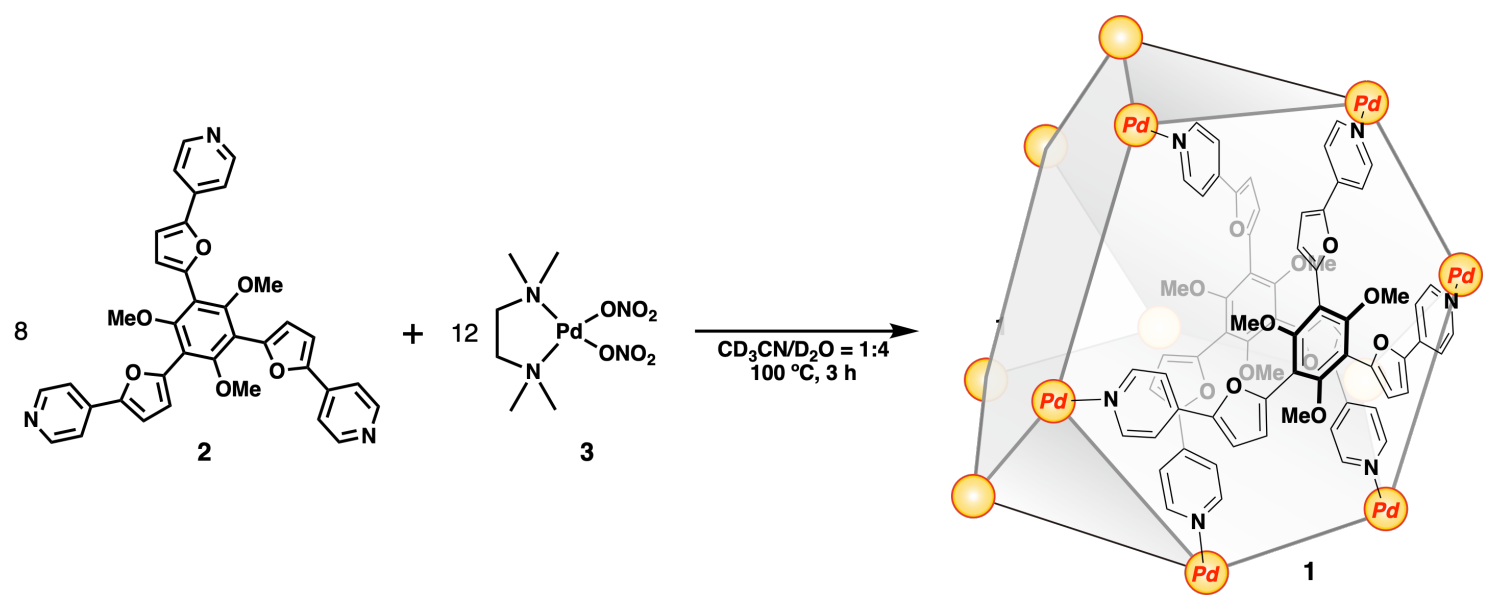

1,3,5-Tris(5-(prydine-4-yl)furan-2-yl)-2,4,6-trimethoxybenzene $\quad(2, \quad 60.0 \quad \mathrm{mg}, \quad 100 \quad \mu \mathrm{mol}) \quad$ and (tmeda) $\mathrm{Pd}\left(\mathrm{ONO}_{2}\right)_{2}(3,52.0 \mathrm{mg}, 150 \mu \mathrm{mol})$ were added in acetonitrile- $d_{3} / \mathrm{D}_{2} \mathrm{O}(5.0 \mathrm{~mL}, 1: 4 \mathrm{v} / \mathrm{v})$. After stirring at $100{ }^{\circ} \mathrm{C}$ for $3 \mathrm{~h}$, a trace amount of insoluble material was filtrated. The quantitative formation of cage 1 was confirmed by ${ }^{1} \mathrm{H}$ NMR spectroscopy.

Physical data of 1: ${ }^{1} \mathrm{H}$ NMR (acetonitrile- $\left.d_{3} / \mathrm{D}_{2} \mathrm{O}(1: 4 \mathrm{v} / \mathrm{v}), 500 \mathrm{MHz}\right): \delta 10.16(\mathrm{~d}, J=5.5 \mathrm{~Hz}, 4 \mathrm{H}, \mathrm{Ar} H$ ), 10.09 (d, $J=6.0 \mathrm{~Hz}, 4 \mathrm{H}, \operatorname{Ar} H), 9.68(\mathrm{~d}, J=6.0 \mathrm{~Hz}, 4 \mathrm{H}, \operatorname{Ar} H), 9.62$ (d, $J=6.0 \mathrm{~Hz}, 4 \mathrm{H}, \operatorname{Ar} H), 9.58$ (d, $J=$ $6.0 \mathrm{~Hz}, 4 \mathrm{H}, \operatorname{Ar} H$ ), 9.54 (d, $J=6.0 \mathrm{~Hz}, 4 \mathrm{H}, \operatorname{Ar} H), 9.50$ (d, $J=6.0 \mathrm{~Hz}, 4 \mathrm{H}, \operatorname{Ar} H), 9.38(\mathrm{~d}, J=6.0 \mathrm{~Hz}, 4 \mathrm{H}$, $\operatorname{Ar} H), 9.35(\mathrm{~d}, J=6.0 \mathrm{~Hz}, 4 \mathrm{H}, \operatorname{Ar} H), 9.24(\mathrm{~d}, J=4.5 \mathrm{~Hz}, 8 \mathrm{H}, \operatorname{Ar} H), 9.11(\mathrm{~d}, J=6.0 \mathrm{~Hz}, 4 \mathrm{H}, \operatorname{Ar} H), 8.21$ (d, $J=5.5 \mathrm{~Hz}, 4 \mathrm{H}, \operatorname{Ar} H), 7.81(\mathrm{~d}, J=5.5 \mathrm{~Hz}, 4 \mathrm{H}, \operatorname{Ar} H), 7.70(\mathrm{~d}, J=5.5 \mathrm{~Hz}, 12 \mathrm{H}, \operatorname{Ar} H), 7.66(\mathrm{~d}, J=5.5 \mathrm{~Hz}$, 4H, $\operatorname{Ar} H), 7.65(\mathrm{~d}, J=5.5 \mathrm{~Hz}, 4 \mathrm{H}, \operatorname{Ar} H), 7.60(\mathrm{~d}, J=5.5 \mathrm{~Hz}, 4 \mathrm{H}, \operatorname{Ar} H), 7.56(\mathrm{~d}, J=5.5 \mathrm{~Hz}, 4 \mathrm{H}, \operatorname{Ar} H), 7.54$ $(\mathrm{d}, J=5.5 \mathrm{~Hz}, 4 \mathrm{H}, \operatorname{Ar} H), 7.45(\mathrm{~d}, J=5.5 \mathrm{~Hz}, 4 \mathrm{H}, \operatorname{Ar} H), 7.17(\mathrm{~d}, J=3.5 \mathrm{~Hz}, 4 \mathrm{H}, \operatorname{Ar} H), 7.11(\mathrm{~d}, J=5.0 \mathrm{~Hz}$, 4H, $\operatorname{Ar} H), 7.07$ (d, $J=3.5 \mathrm{~Hz}, 4 \mathrm{H}, \operatorname{Ar} H), 7.05(\mathrm{~d}, J=4.0 \mathrm{~Hz}, 4 \mathrm{H}, \operatorname{Ar} H), 6.78(\mathrm{~d}, J=3.5 \mathrm{~Hz}, 4 \mathrm{H}, \operatorname{Ar} H), 6.77$ (d, $J=4.0 \mathrm{~Hz}, 4 \mathrm{H}, \operatorname{Ar} H), 6.61(\mathrm{~d}, J=3.5 \mathrm{~Hz}, 4 \mathrm{H}, \operatorname{Ar} H), 5.88(\mathrm{~d}, J=3.5 \mathrm{~Hz}, 4 \mathrm{H}, \operatorname{Ar} H), 5.80(\mathrm{~d}, J=3.0 \mathrm{~Hz}$, $4 \mathrm{H}, \operatorname{Ar} H), 5.36(\mathrm{~d}, J=3.0 \mathrm{~Hz}, 4 \mathrm{H}, \operatorname{Ar} H), 5.33(\mathrm{~d}, J=3.0 \mathrm{~Hz}, 4 \mathrm{H}, \operatorname{Ar} H), 4.92(\mathrm{~d}, J=3.5 \mathrm{~Hz}, 4 \mathrm{H}, \operatorname{Ar} H), 4.46$ (d, $J=3.0 \mathrm{~Hz}, 4 \mathrm{H}, \mathrm{Ar} H$ ), 3.92 (s, 12H, -OMe), 3.82 (s, 12H, -OMe), 3.62 (s, 12H, -OMe), 3.49 (s, 12H, $\mathrm{OMe}$ ), 3.49-3.23 (br, 40H, $-\mathrm{CH}_{2}-$ ), 3.27 (s, $12 \mathrm{H},-\mathrm{OMe}$ ), 3.26 (s, $12 \mathrm{H}, \mathrm{CH}_{3}$ ), 3.18 (s, 36H, $\mathrm{CH}_{3}$ ), 3.07 (br, $\left.8 \mathrm{H},-\mathrm{CH}_{2}-\right), 3.06\left(\mathrm{~s}, 12 \mathrm{H}, \mathrm{CH}_{3}\right), 3.04\left(\mathrm{~s}, 12 \mathrm{H}, \mathrm{CH}_{3}\right), 2.97\left(\mathrm{~s}, 24 \mathrm{H}, \mathrm{CH}_{3}\right), 2.94\left(\mathrm{~s}, 24 \mathrm{H}, \mathrm{CH}_{3}\right), 2.92(\mathrm{~s}, 12 \mathrm{H}$, $\mathrm{CH}_{3}$ ), 2.91 (s, $12 \mathrm{H}, \mathrm{CH}_{3}$ ), 2.05 (s, $12 \mathrm{H},-\mathrm{OMe}$ ); ${ }^{13} \mathrm{C}$ NMR (acetonitrile- $d_{3} / \mathrm{D}_{2} \mathrm{O}(1: 4 \mathrm{v} / \mathrm{v}), 125 \mathrm{MHz}$ ): $\delta 157.1$ (C), $157.0(C), 156.9(C), 156.7(C), 155.8(C), 153.2(\mathrm{CH}), 152.6(\mathrm{CH}), 152.5(\mathrm{CH}), 151.9(\mathrm{CH}), 151.8$ $(\mathrm{CH}), 151.6(\mathrm{CH}), 151.1(\mathrm{CH}), 151.1(\mathrm{CH}), 150.7(\mathrm{CH}), 150.0(\mathrm{C}), 149.5(\mathrm{C}), 149.0(\mathrm{C}), 148.7(\mathrm{C}), 148.5$ (C), $148.1(C), 147.9(C), 147.6(C), 147.5(C), 147.4(C), 147.1(C), 140.4(C), 140.3(C), 139.6(C), 139.2$ (C), $121.2(\mathrm{CH}), 121.0(\mathrm{CH}), 120.9(\mathrm{CH}), 120.7(\mathrm{CH}), 120.6(\mathrm{CH}), 120.3(\mathrm{CH}), 120.2(\mathrm{CH}), 119.6(\mathrm{CH})$, 116.7 (C), $116.1(\mathrm{CH}), 115.8(\mathrm{C}), 115.8(\mathrm{CH}), 115.6(\mathrm{CH}), 115.3(\mathrm{C}), 115.3(\mathrm{CH}), 114.9(\mathrm{C}), 114.9(\mathrm{CH})$, $114.4(\mathrm{CH}), 114.3(\mathrm{CH}), 114.1(\mathrm{CH}), 113.5(\mathrm{CH}), 112.5(\mathrm{CH}), 112.1(\mathrm{CH}), 111.9(\mathrm{C}), 111.8(\mathrm{CH}), 63.4(-$ $\mathrm{OMe}), 63.2\left(-\mathrm{CH}_{2}-\right), 63.1\left(-\mathrm{CH}_{2}-\right), 62.9\left(-\mathrm{CH}_{2}-\right), 62.5\left(-\mathrm{CH}_{2}-\right), 61.5(-\mathrm{OMe}), 61.1(-\mathrm{OMe}), 61.1(-\mathrm{OMe})$, 60.9 (-OMe), $59.2(-\mathrm{OMe}), 51.4\left(\mathrm{CH}_{3}\right), 51.2\left(\mathrm{CH}_{3}\right), 51.0\left(\mathrm{CH}_{3}\right), 50.9\left(\mathrm{CH}_{3}\right), 50.9\left(\mathrm{CH}_{3}\right), 50.7\left(\mathrm{CH}_{3}\right), 50.7$ $\left(\mathrm{CH}_{3}\right), 50.5\left(\mathrm{CH}_{3}\right), 50.4\left(\mathrm{CH}_{3}\right)$. DOSY-NMR (acetonitrile- $\left.d_{3} / \mathrm{D}_{2} \mathrm{O}(1: 4 \mathrm{v} / \mathrm{v}), \mathrm{m}^{2} / \mathrm{s}\right): D=1.26 \times 10^{-10} . \mathrm{IR}$ $\left(\right.$ ATR, $\mathrm{cm}^{-1}$ ): 2968, 2886, 2367, 2360, 2343, 2331, 1341, 1044, 811, 719, 656, 627, 560. m.p.: $>155^{\circ} \mathrm{C}$ 
(decomposed). HR-MS (ESI): calcd. for $\left[\left(\mathrm{C}_{36} \mathrm{H}_{27} \mathrm{~N}_{3} \mathrm{O}_{6}\right)_{8}\left(\mathrm{C}_{6} \mathrm{H}_{16} \mathrm{~N}_{2} \mathrm{Pd}\right)_{12}\left(\mathrm{BF}_{4}\right)_{16}\right]^{8+}: 1105.1267$, found: 1105.1280.
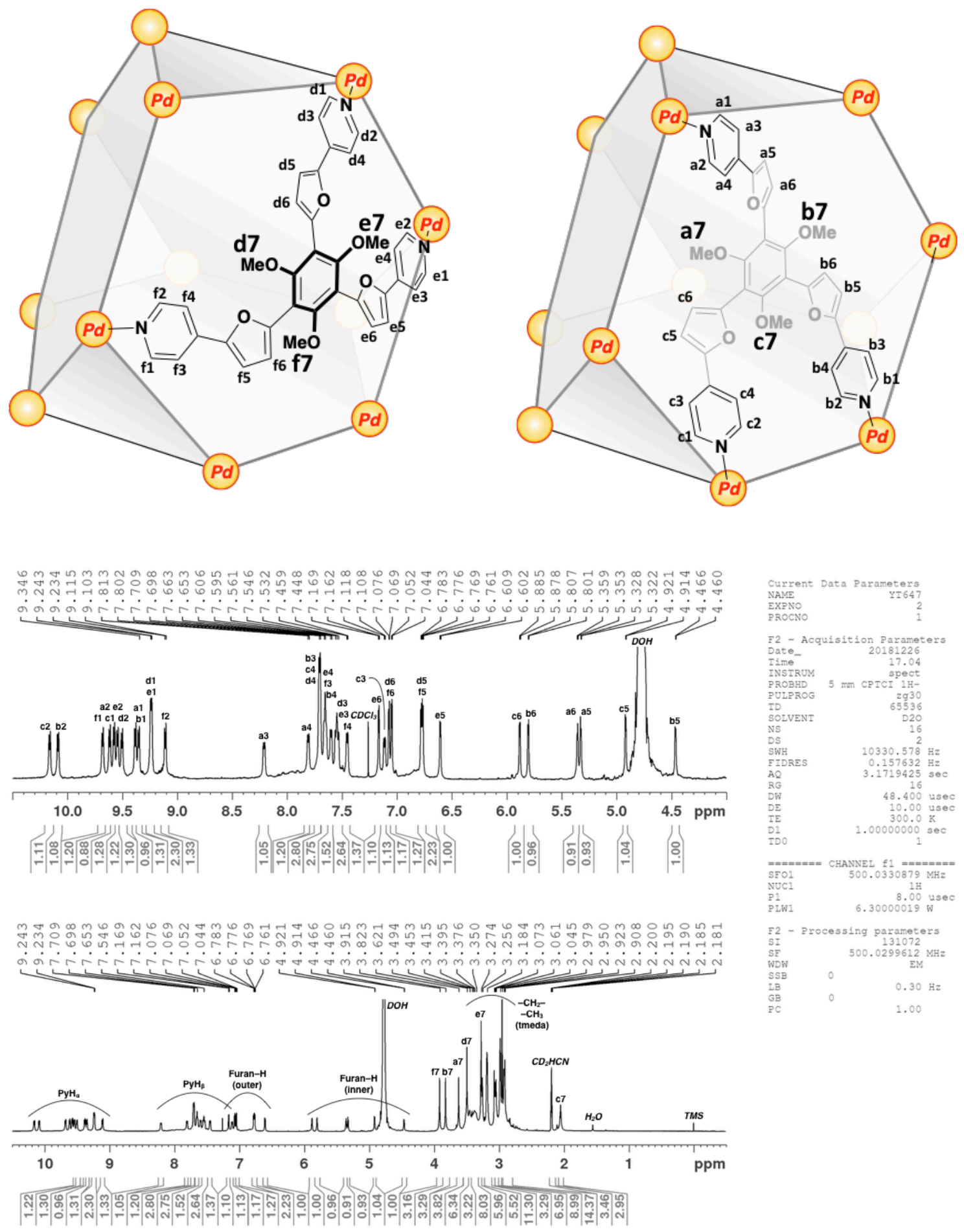

Figure S6. ${ }^{1} \mathrm{H}$ NMR spectrum $\left(500 \mathrm{MHz}, 300 \mathrm{~K}\right.$, acetonitrile- $\left.d_{3} / \mathrm{D}_{2} \mathrm{O}(1: 4 \mathrm{v} / \mathrm{v})\right)$ of $\mathbf{1}$. 

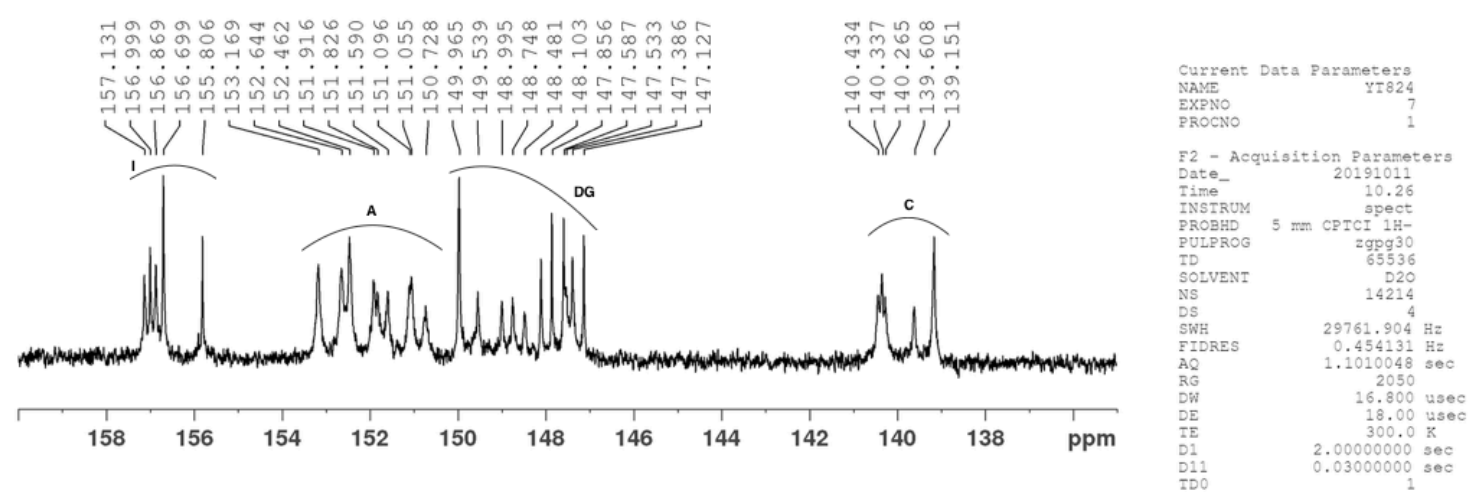

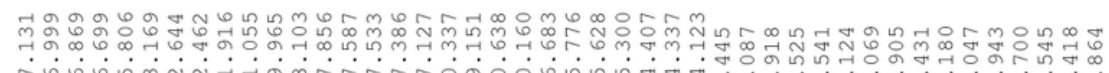
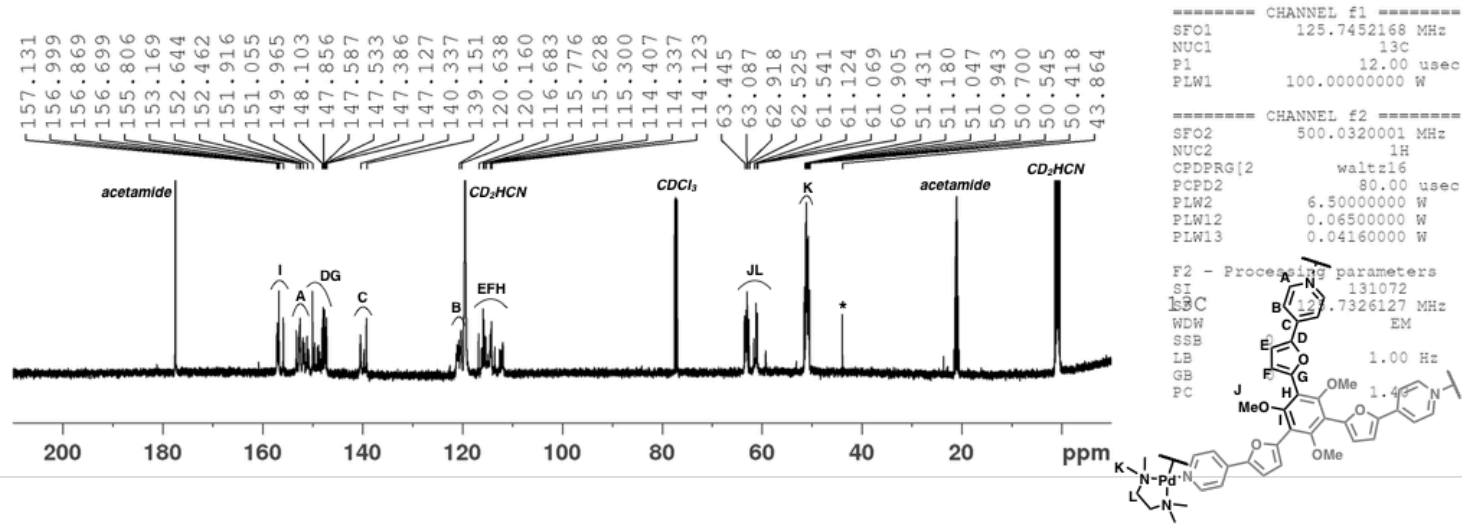

Figure S7. ${ }^{13} \mathrm{C}$ NMR spectrum $\left(125 \mathrm{MHz}, 300 \mathrm{~K}\right.$, acetonitrile- $\left.d_{3} / \mathrm{D}_{2} \mathrm{O}(1: 4 \mathrm{v} / \mathrm{v})\right)$ of $\mathbf{1}$.
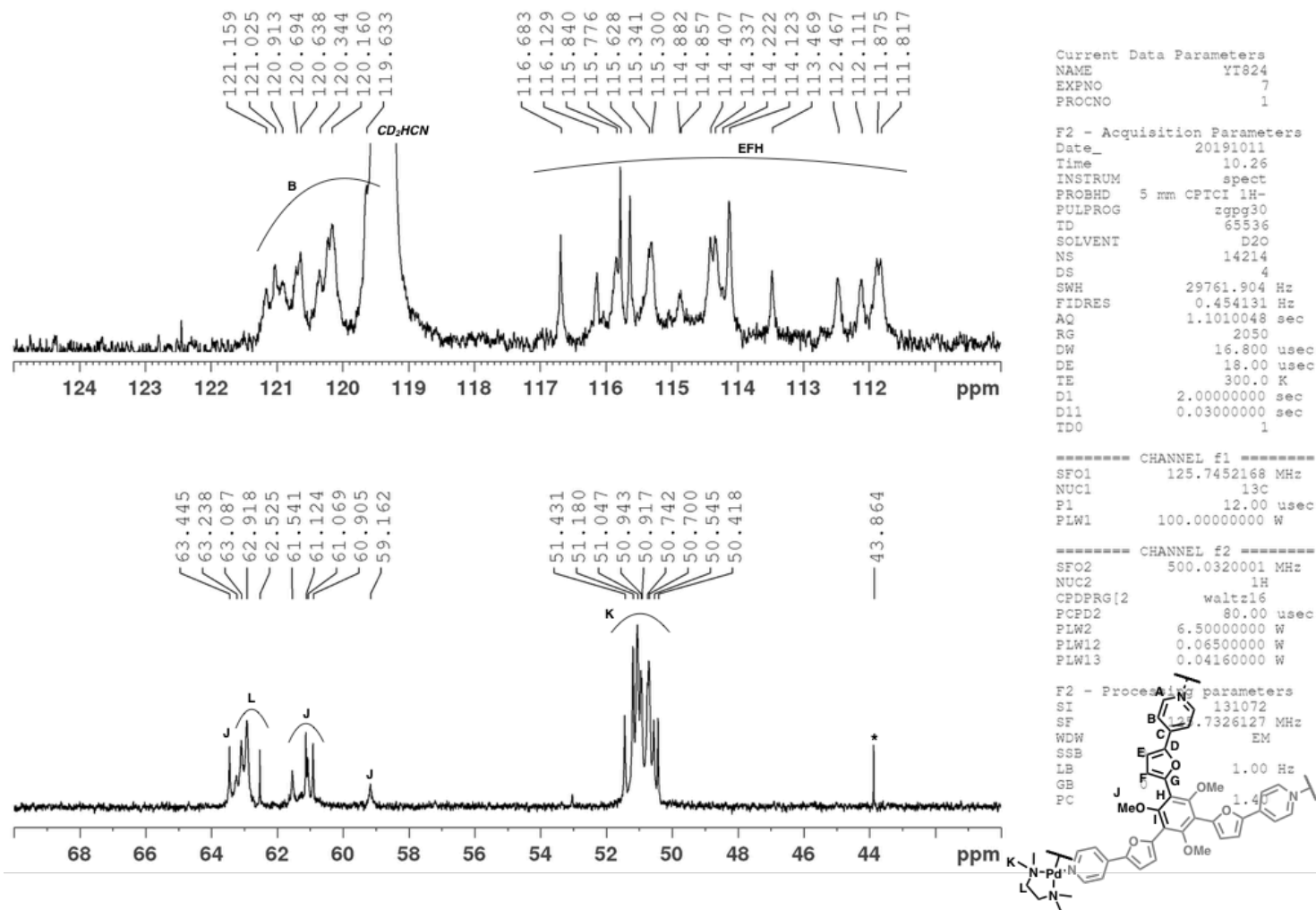

Figure S8. ${ }^{13} \mathrm{C}$ NMR spectrum $\left(125 \mathrm{MHz}, 300 \mathrm{~K}\right.$, acetonitrile- $\left.d_{3} / \mathrm{D}_{2} \mathrm{O}(1: 4 \mathrm{v} / \mathrm{v})\right)$ of $\mathbf{1}$. 

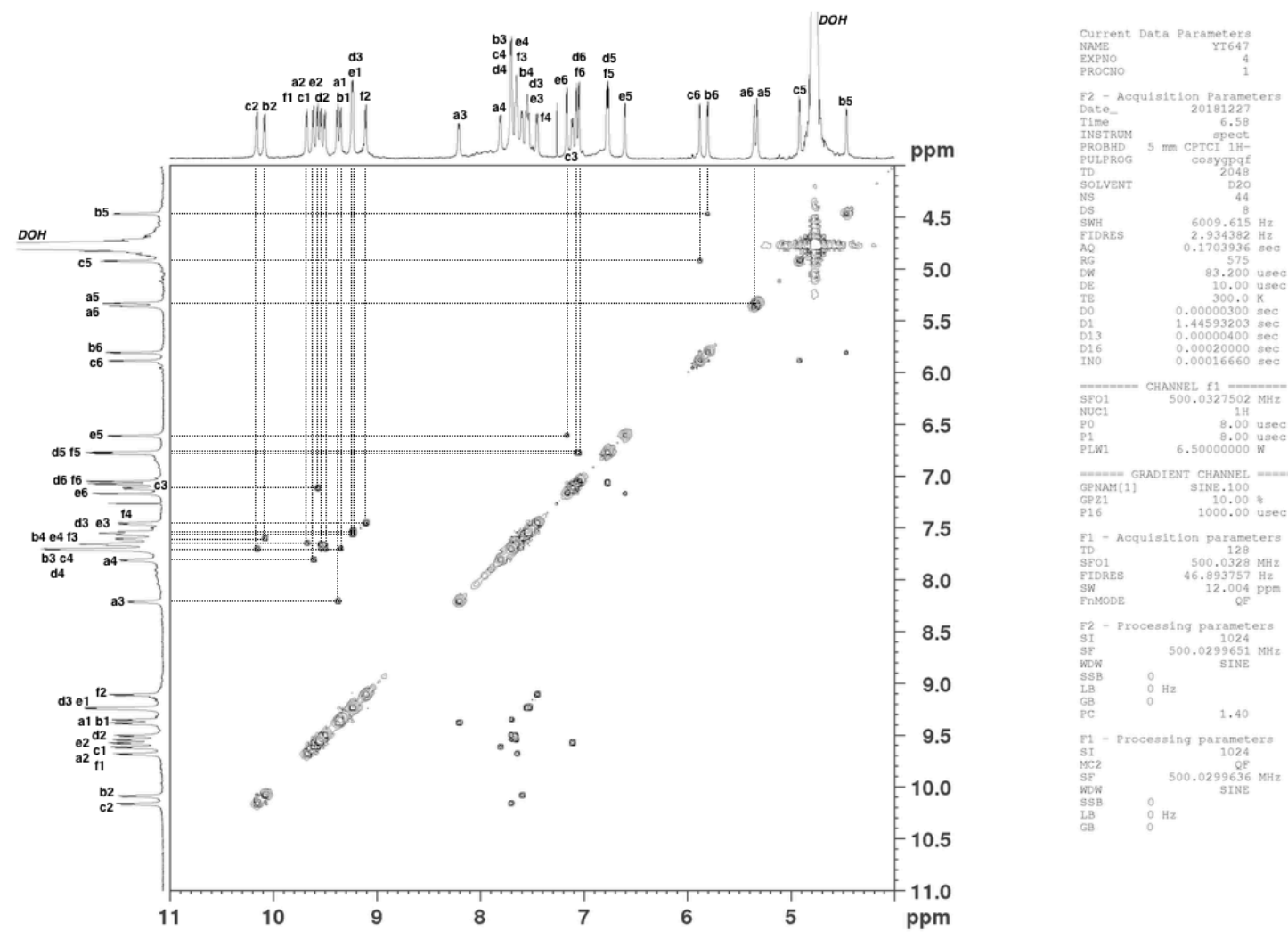

Figure S9. ${ }^{1} \mathrm{H}-{ }^{1} \mathrm{H}$ COSY NMR spectrum $\left(500 \mathrm{MHz}, 300 \mathrm{~K}\right.$, acetonitrile- $\left.d_{3} / \mathrm{D}_{2} \mathrm{O}(1: 4 \mathrm{v} / \mathrm{v})\right)$ of $\mathbf{1}$.
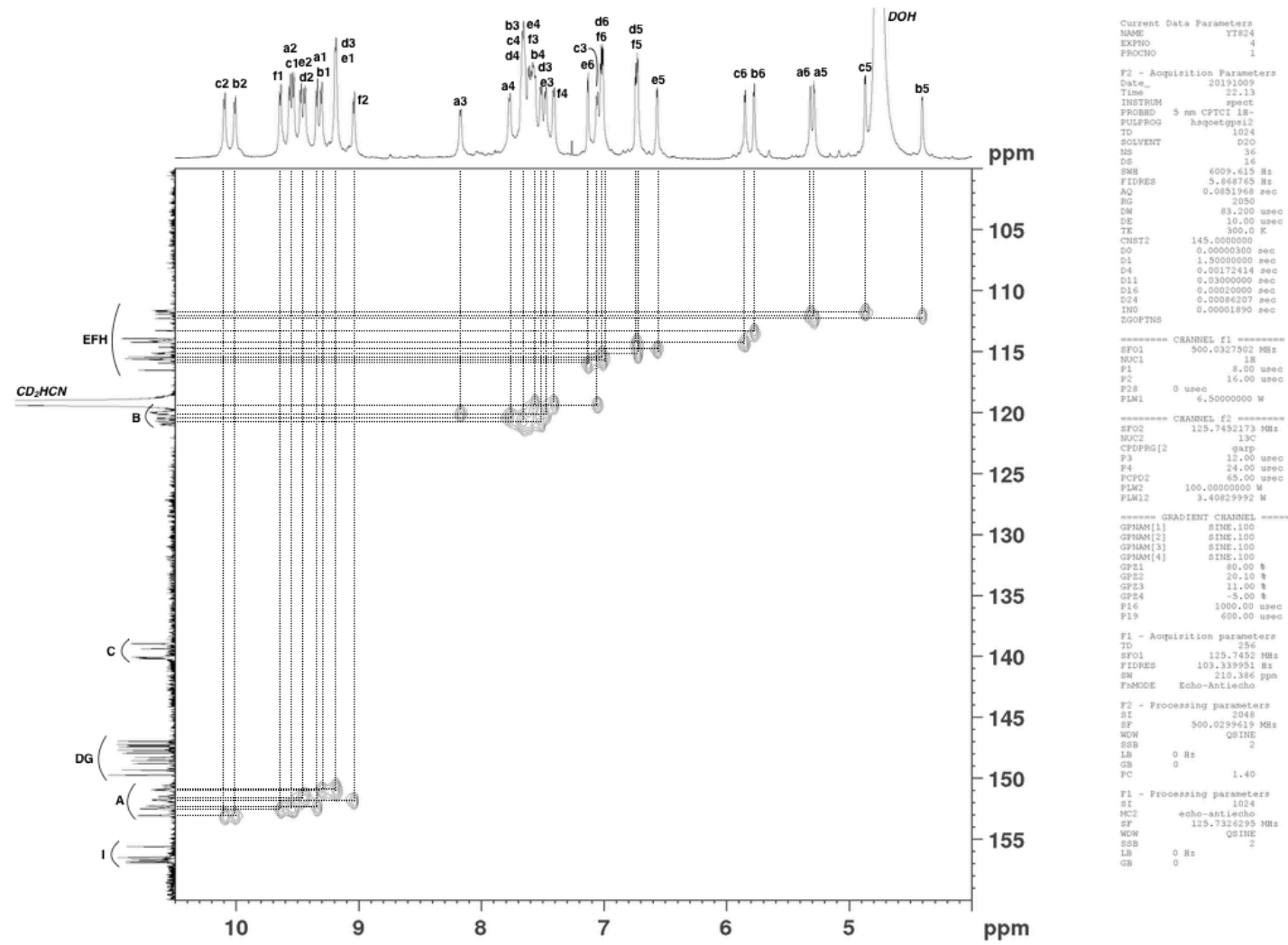

Figure S10. ${ }^{1} \mathrm{H}-{ }^{13} \mathrm{C}$ HSQC NMR spectrum $\left(500 \mathrm{MHz}, 300 \mathrm{~K}\right.$, acetonitrile- $\left.d_{3} / \mathrm{D}_{2} \mathrm{O}(1: 4 \mathrm{v} / \mathrm{v})\right)$ of $\mathbf{1}$. 


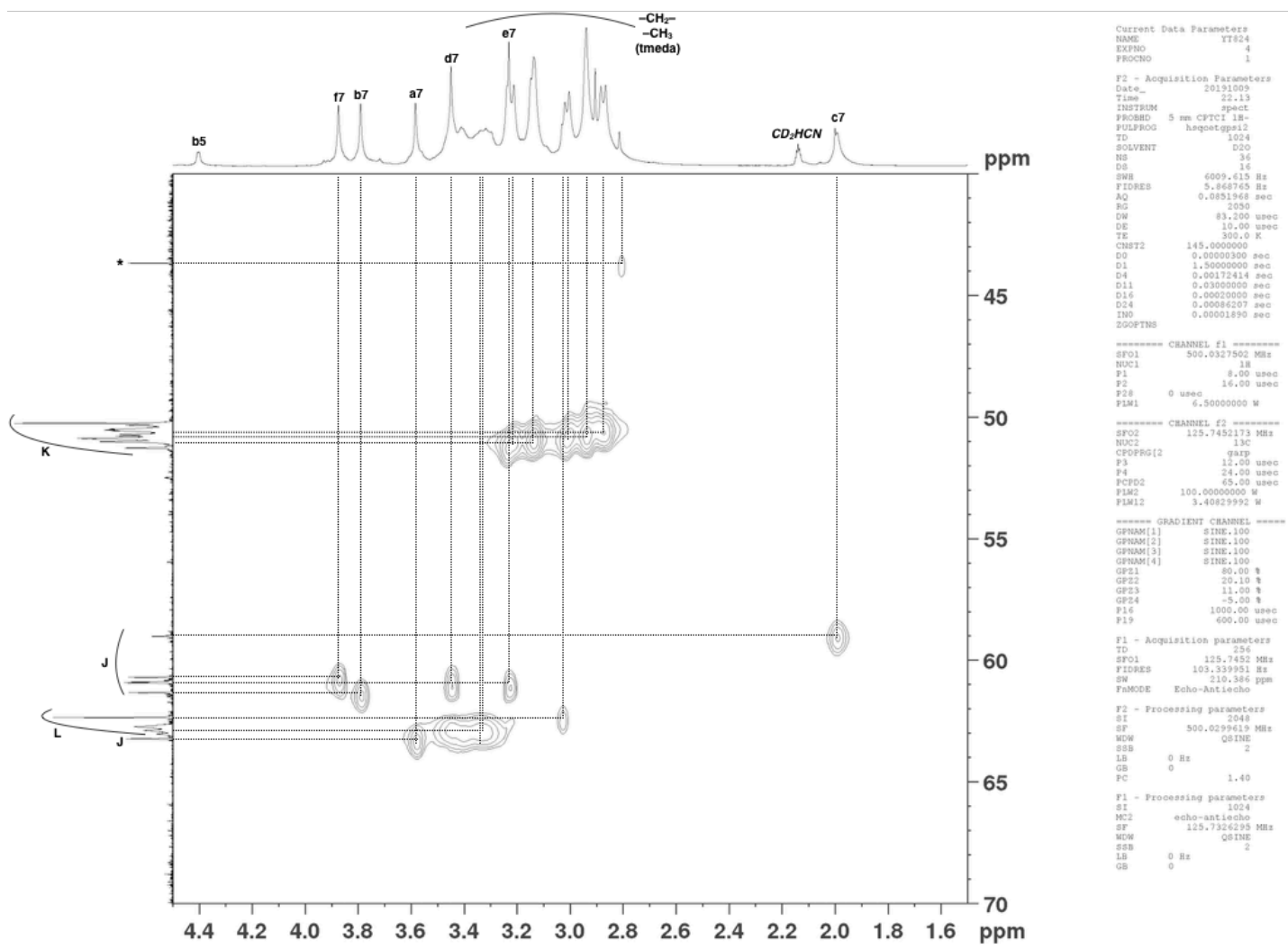

Figure S11. ${ }^{1} \mathrm{H}-{ }^{13} \mathrm{C}$ HSQC NMR spectrum $\left(500 \mathrm{MHz}, 300 \mathrm{~K}\right.$, acetonitrile- $\left.d_{3} / \mathrm{D}_{2} \mathrm{O}(1: 4 \mathrm{v} / \mathrm{v})\right)$ of $\mathbf{1}$.
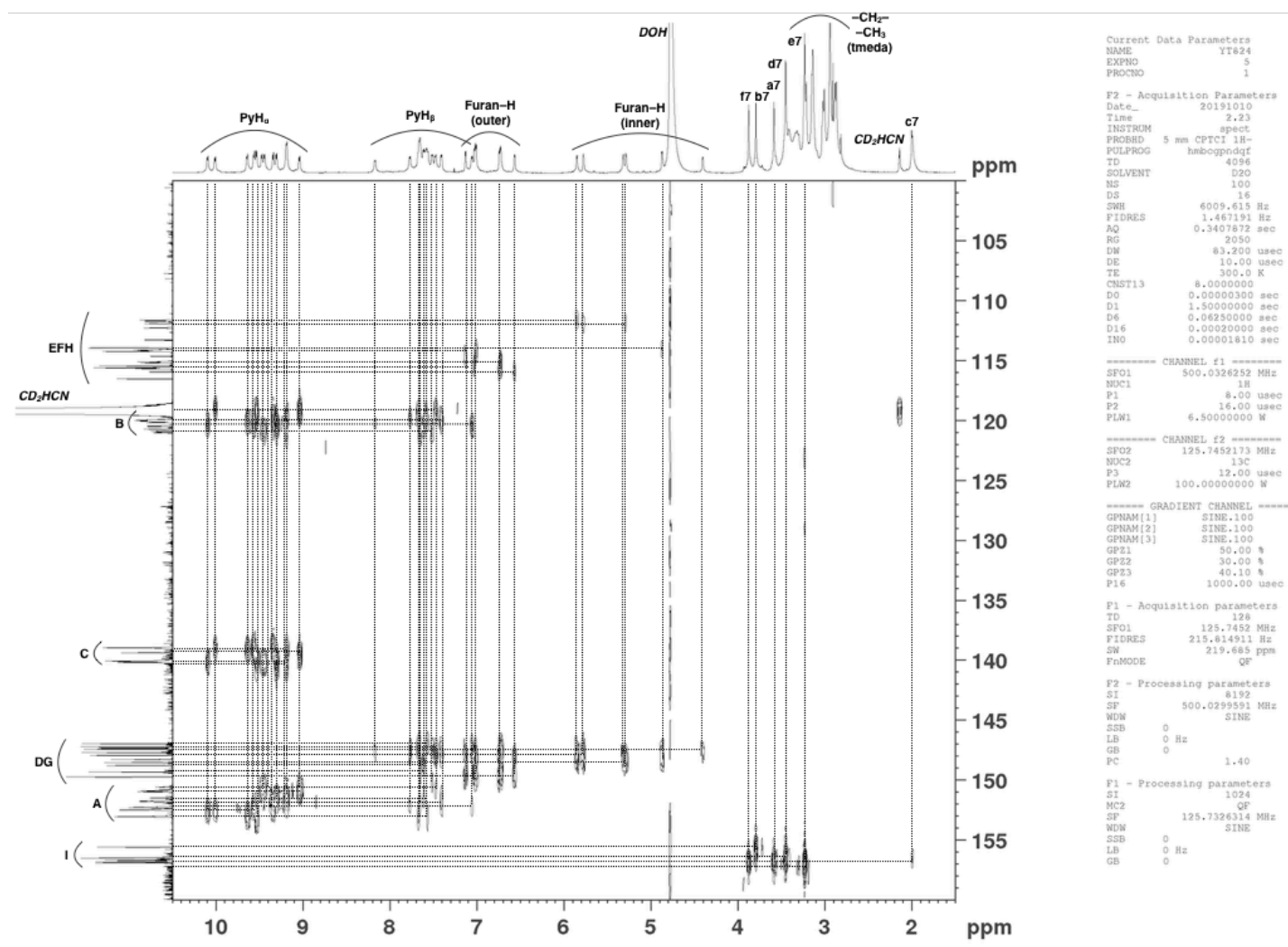

Figure S12. ${ }^{1} \mathrm{H}-{ }^{13} \mathrm{C}$ HMBC NMR spectrum $\left(500 \mathrm{MHz}, 300 \mathrm{~K}\right.$, acetonitrile- $\left.d_{3} / \mathrm{D}_{2} \mathrm{O}(1: 4 \mathrm{v} / \mathrm{v})\right)$ of $\mathbf{1}$. 


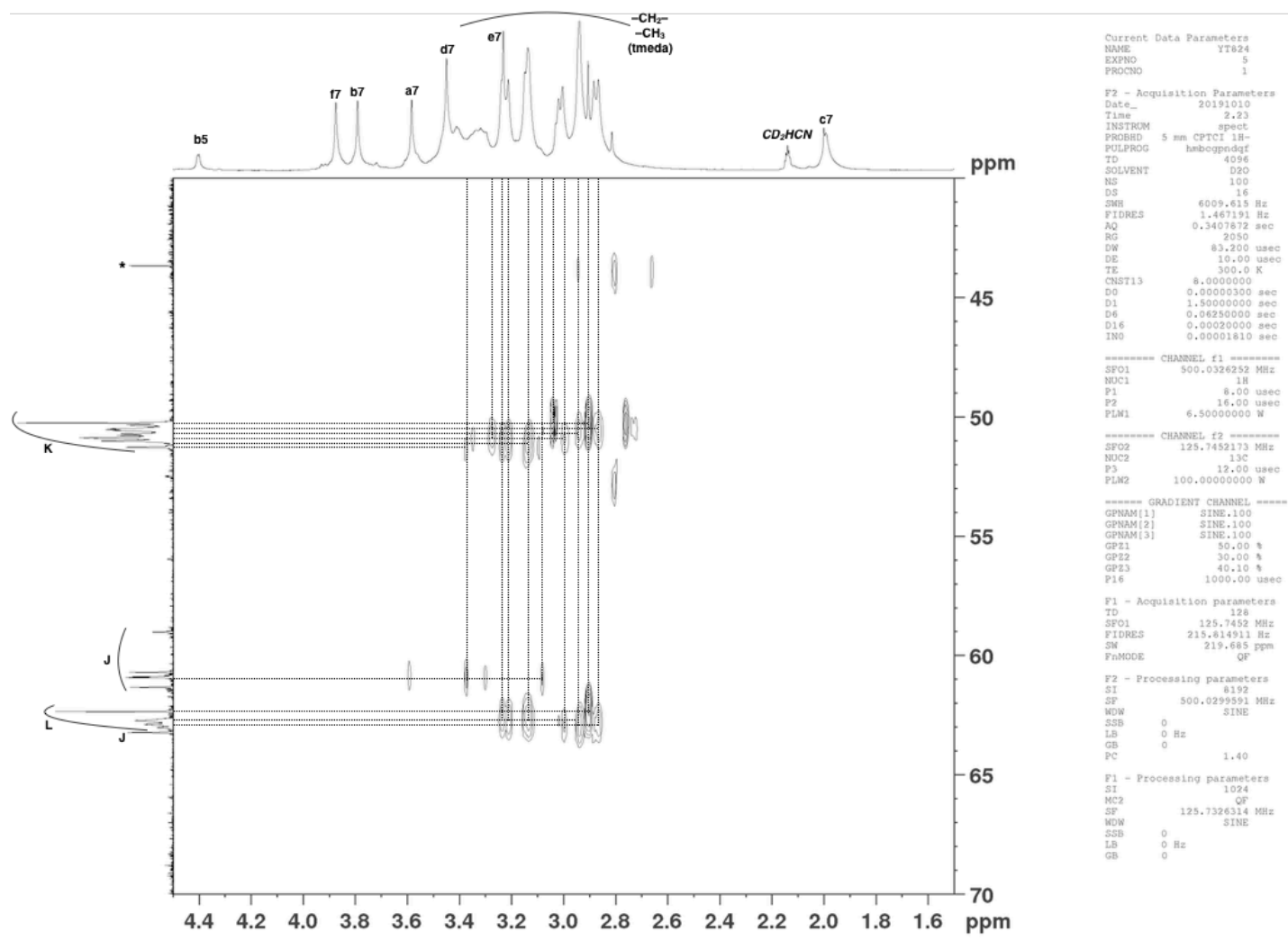

Figure S13. ${ }^{1} \mathrm{H}^{13}{ }^{13} \mathrm{C} \mathrm{HMBC}$ NMR spectrum $\left(500 \mathrm{MHz}, 300 \mathrm{~K}\right.$, acetonitrile- $\left.d_{3} / \mathrm{D}_{2} \mathrm{O}(1: 4 \mathrm{v} / \mathrm{v})\right)$ of $\mathbf{1}$.
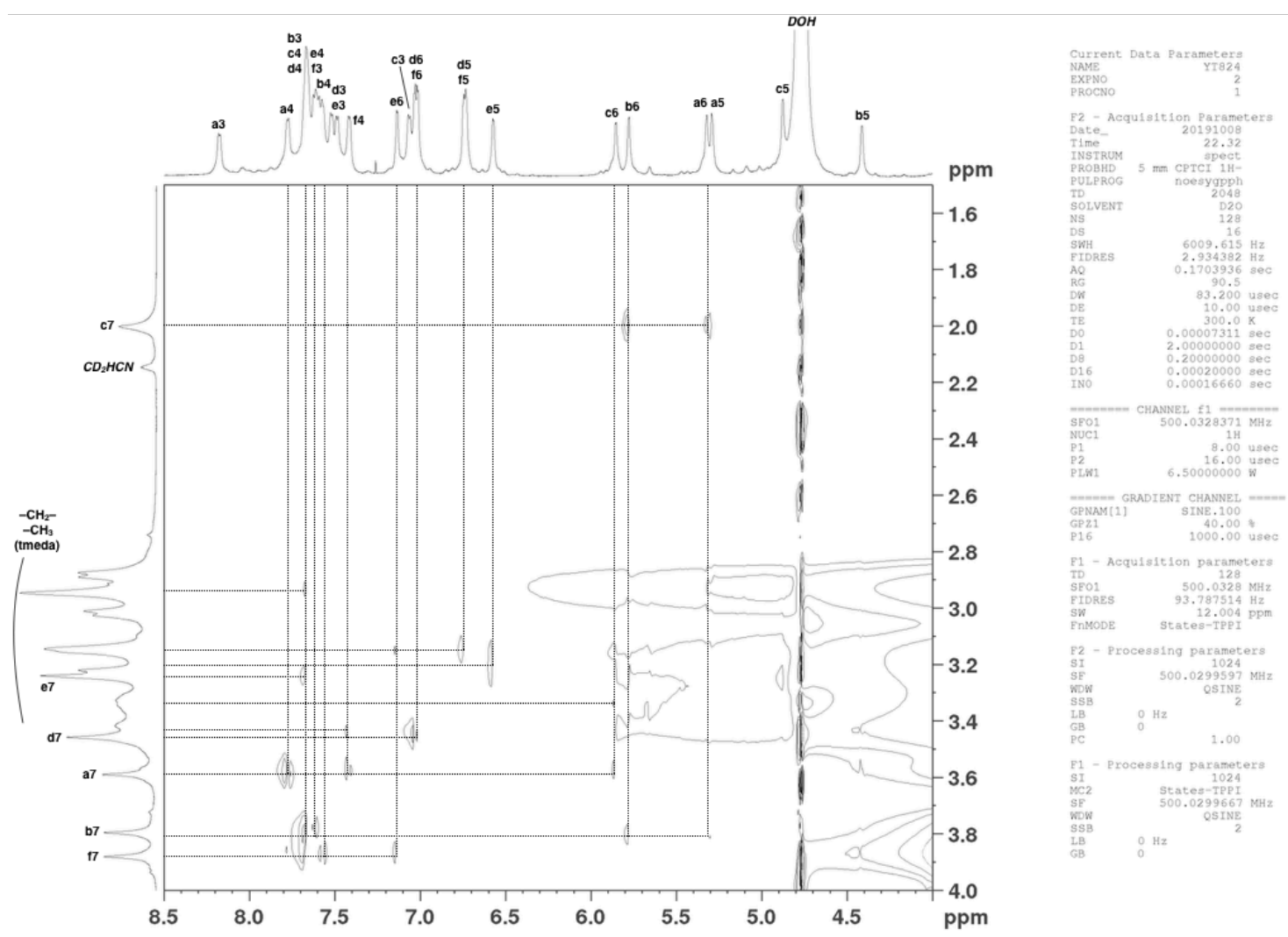

Figure S14. ${ }^{1} \mathrm{H}-{ }^{1} \mathrm{H}$ NOESY NMR spectrum $\left(500 \mathrm{MHz}, 300 \mathrm{~K}\right.$, acetonitrile- $\left.d_{3} / \mathrm{D}_{2} \mathrm{O}(1: 4 \mathrm{v} / \mathrm{v})\right)$ of $\mathbf{1}$. 


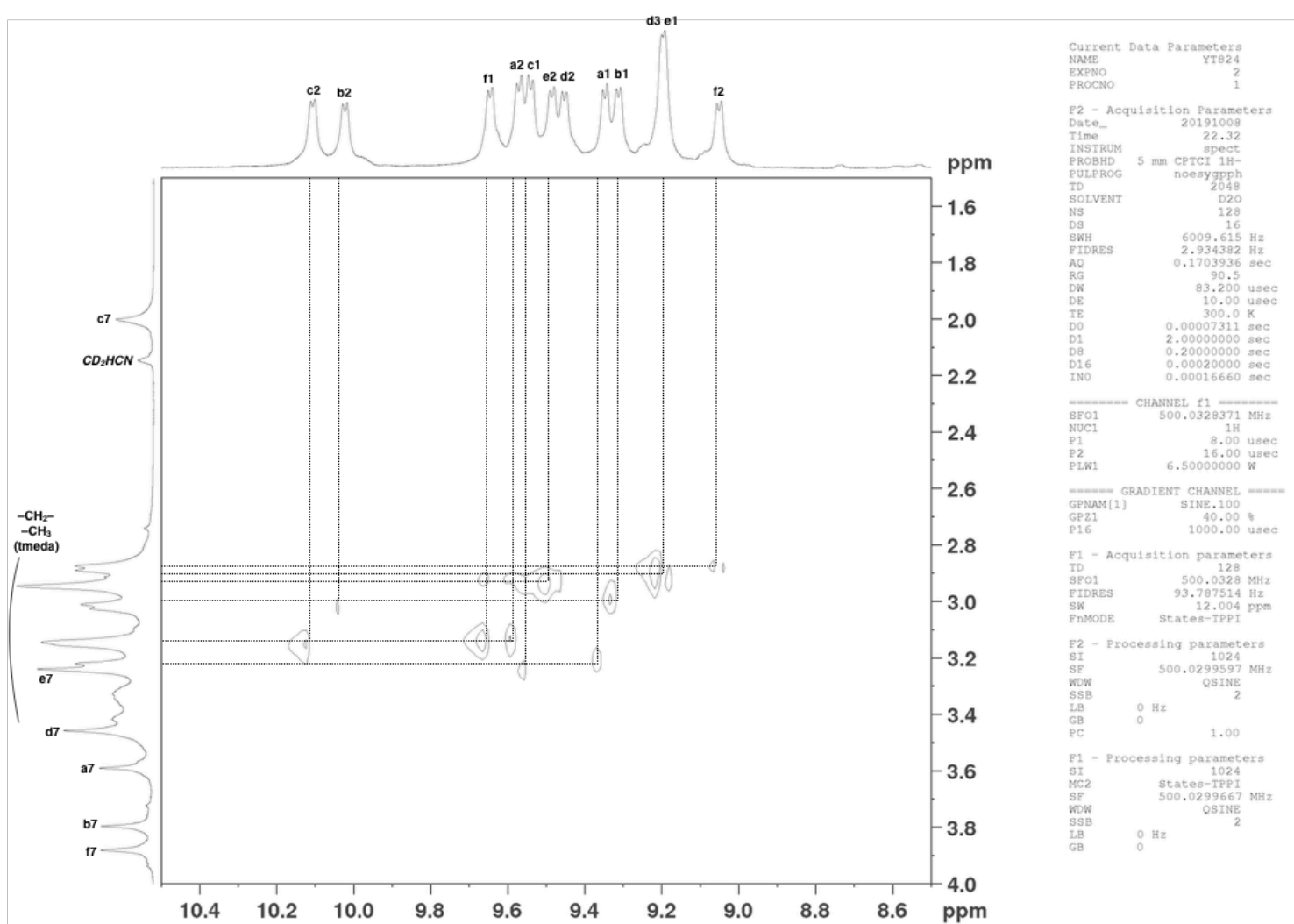

Figure S15. ${ }^{1} \mathrm{H}-{ }^{1} \mathrm{H}$ NOESY NMR spectrum $\left(500 \mathrm{MHz}, 300 \mathrm{~K}\right.$, acetonitrile- $\left.d_{3} / \mathrm{D}_{2} \mathrm{O}(1: 4 \mathrm{v} / \mathrm{v})\right)$ of $\mathbf{1}$.

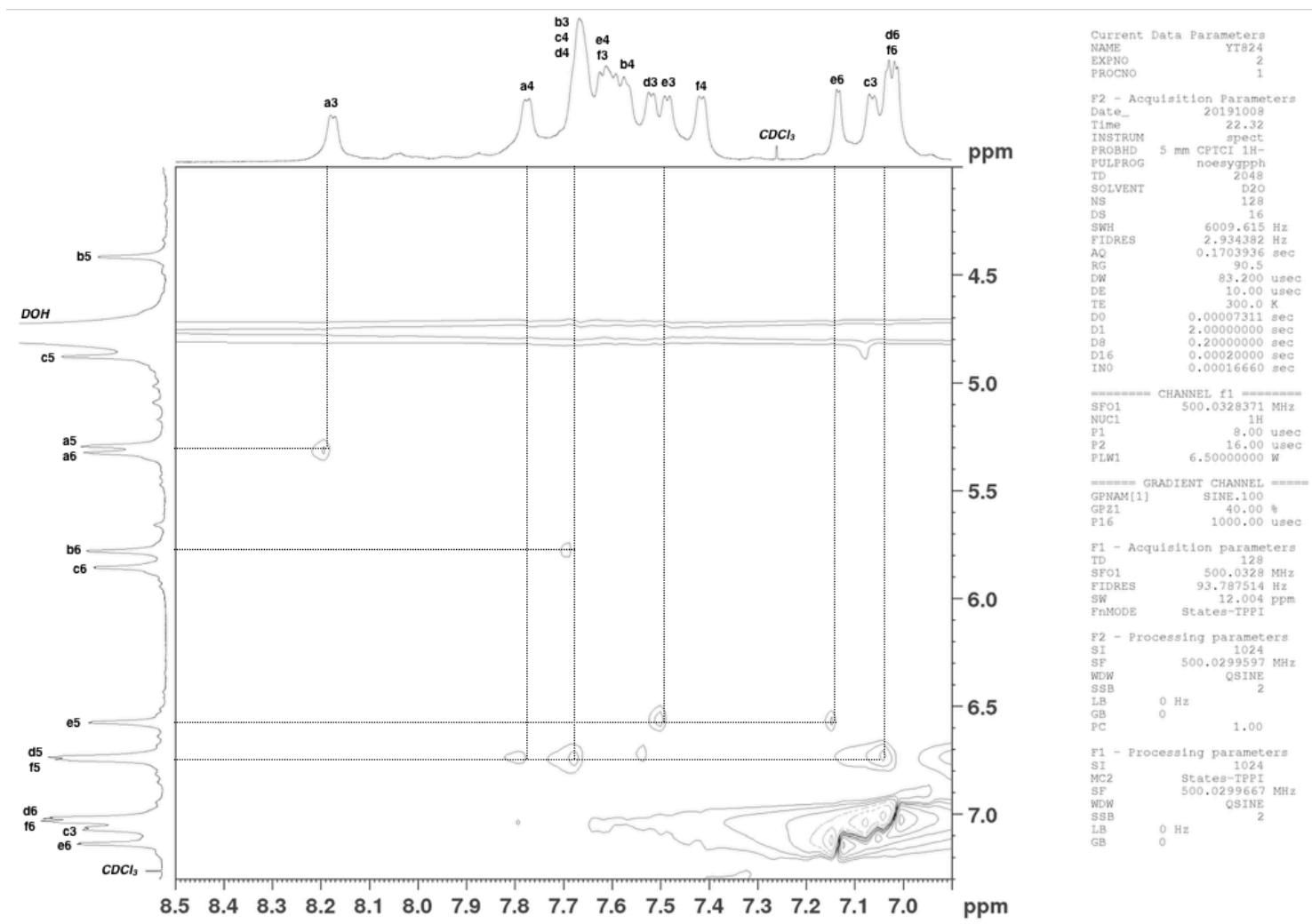

Figure S16. ${ }^{1} \mathrm{H}-{ }^{1} \mathrm{H}$ NOESY NMR spectrum $\left(500 \mathrm{MHz}, 300 \mathrm{~K}\right.$, acetonitrile- $\left.d_{3} / \mathrm{D}_{2} \mathrm{O}(1: 4 \mathrm{v} / \mathrm{v})\right)$ of $\mathbf{1}$. 


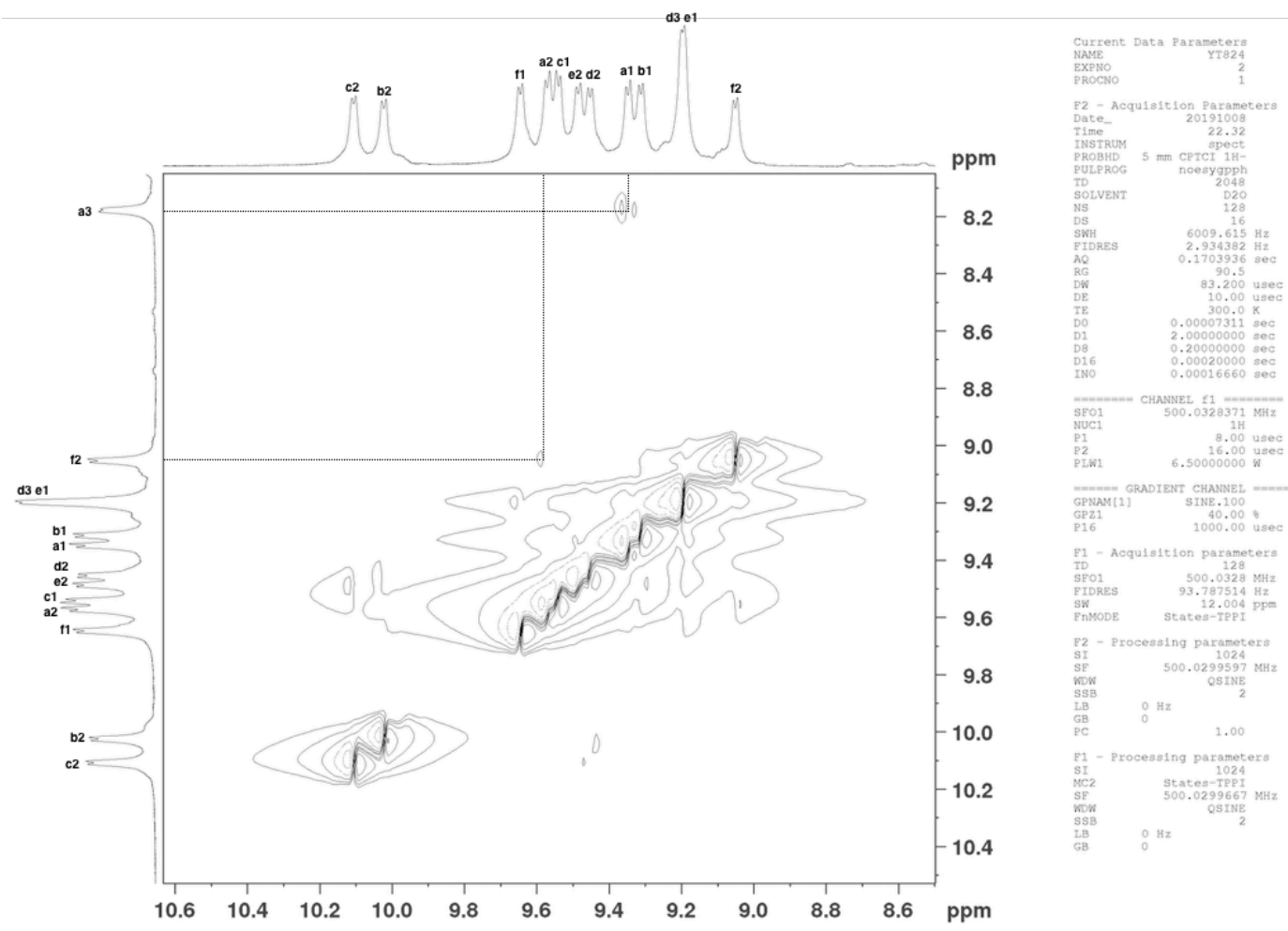

Figure S17. ${ }^{1} \mathrm{H}-{ }^{1} \mathrm{H}$ NOESY NMR spectrum $\left(500 \mathrm{MHz}, 300 \mathrm{~K}\right.$, acetonitrile- $\left.d_{3} / \mathrm{D}_{2} \mathrm{O}(1: 4 \mathrm{v} / \mathrm{v})\right)$ of $\mathbf{1}$.

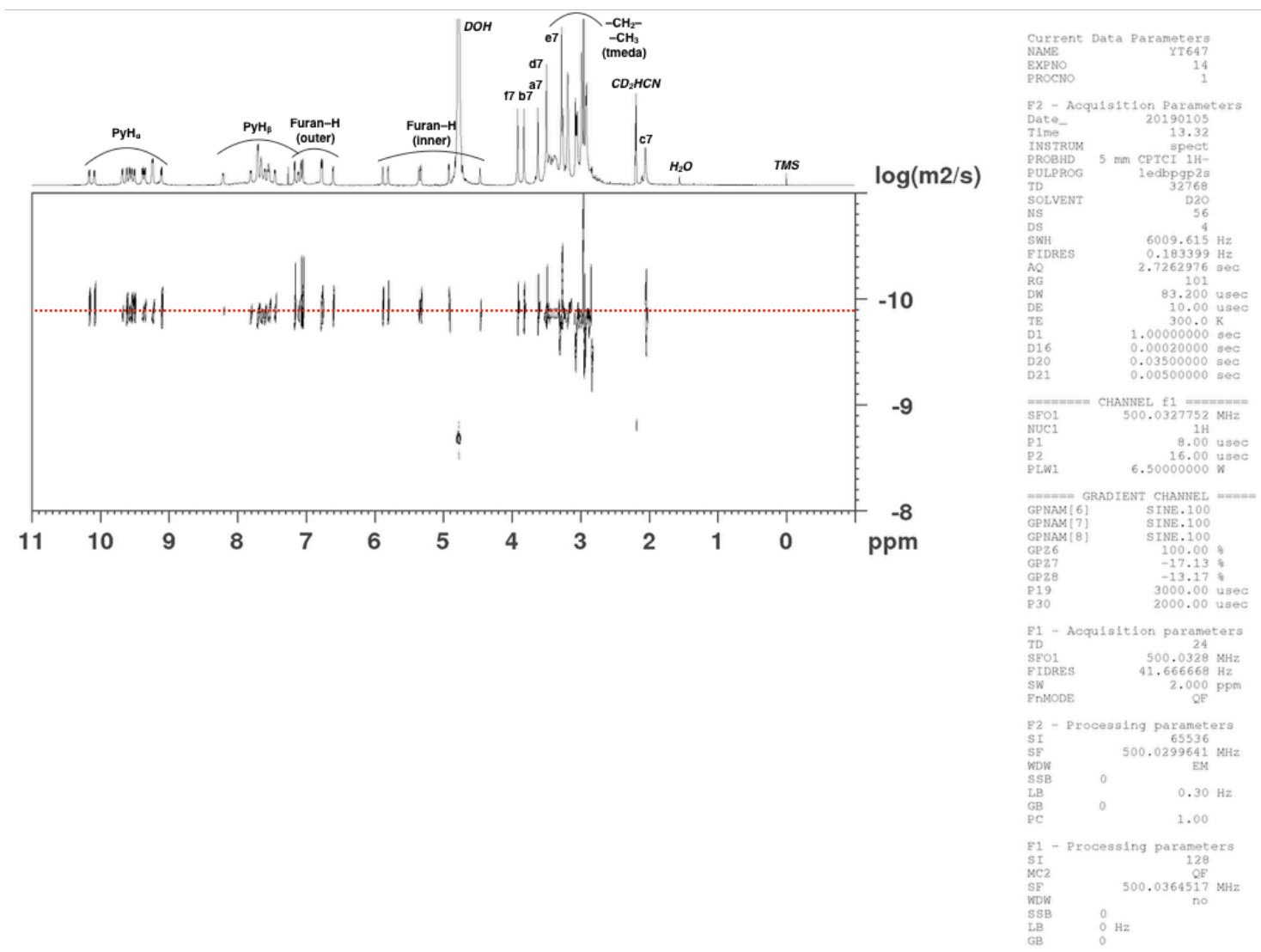

Figure S18. ${ }^{1} \mathrm{H}$ DOSY NMR spectrum $\left(500 \mathrm{MHz}, 300 \mathrm{~K}\right.$, acetonitrile- $\left.d_{3} / \mathrm{D}_{2} \mathrm{O}(1: 4 \mathrm{v} / \mathrm{v})\right)$ of $\mathbf{1}$. 

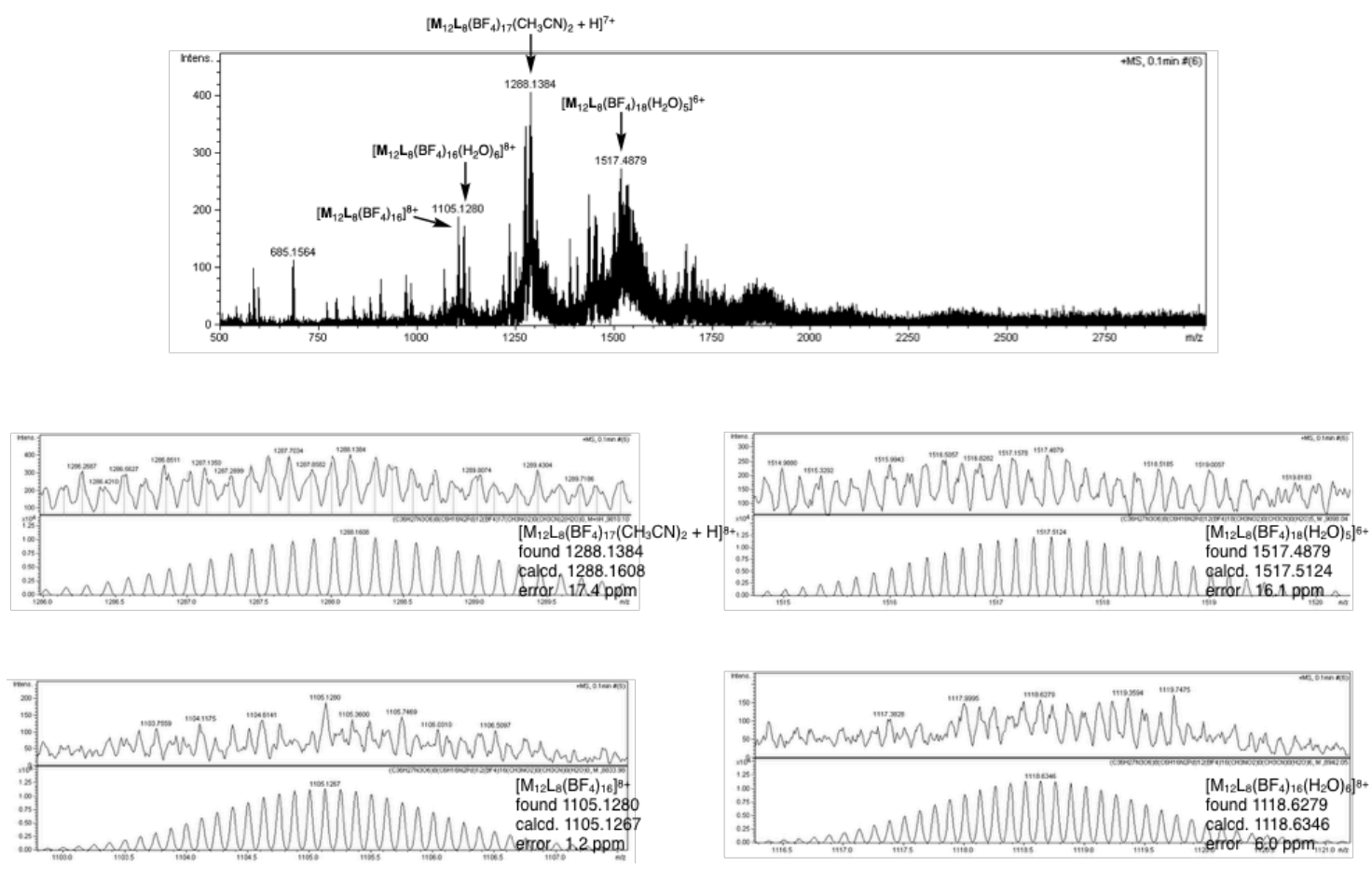

Figure S19. ESI-MS spectra of 1. 


\section{Inclusion experiments}

\section{General procedure}
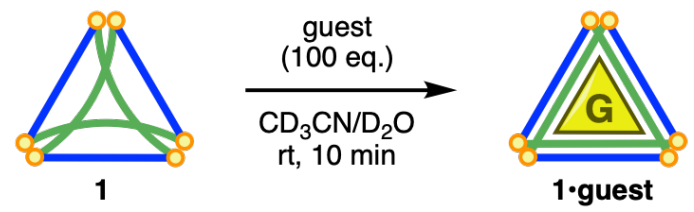

Dried powder of cage $1(18.0 \mathrm{mg}, 2.0 \mu \mathrm{mol})$ was dissolved in acetonitrile- $d_{3} / \mathrm{D}_{2} \mathrm{O}(800 \mu \mathrm{L}, 1: 4 \mathrm{v} / \mathrm{v})$. Guest molecule (100 eq.) was added to the solution and stirred at room temperature for $10 \mathrm{~min}$. The formation of the inclusion complex was confirmed by ${ }^{1} \mathrm{H}$ NMR spectroscopy. The yields of the inclusion complex were determined by comparison of the integral ratio between the signals from empty cage $\mathbf{1}$ and the guest included cage $\mathbf{1} \cdot \mathbf{G}$.

Physical data of $1 \cdot \mathbf{C C l}_{4}:{ }^{1} \mathrm{H}$ NMR (acetonitrile- $\left.d_{3} / \mathrm{D}_{2} \mathrm{O}(1: 4 \mathrm{v} / \mathrm{v}), 500 \mathrm{MHz}\right): \delta 10.00(\mathrm{~d}, J=6.0 \mathrm{~Hz}, 4 \mathrm{H}$, $\operatorname{Ar} H), 9.90$ (d, $J=6.0 \mathrm{~Hz}, 4 \mathrm{H}, \operatorname{Ar} H), 9.63$ (d, J=6.0 Hz, 4H, ArH), 9.58 (d, J=5.5 Hz, 4H, ArH), 9.50 (d, $J=5.5 \mathrm{~Hz}, 4 \mathrm{H}, \operatorname{Ar} H), 9.38(\mathrm{~d}, J=6.0 \mathrm{~Hz}, 4 \mathrm{H}, \operatorname{Ar} H), 9.32(\mathrm{~d}, J=5.5 \mathrm{~Hz}, 8 \mathrm{H}, \operatorname{Ar} H), 9.30(\mathrm{~d}, J=5.5 \mathrm{~Hz}$, 4H, ArH), 9.23 (d, J=6.0 Hz, 4H, ArH), 9.14 (d, J=6.5 Hz, 4H, ArH), 9.03 (d, J=6.0 Hz, 4H, ArH), 8.06 $(\mathrm{d}, J=5.0 \mathrm{~Hz}, 4 \mathrm{H}, \operatorname{Ar} H), 7.87(\mathrm{~d}, J=5.5 \mathrm{~Hz}, 4 \mathrm{H}, \operatorname{Ar} H), 7.76(\mathrm{~d}, J=5.5 \mathrm{~Hz}, 4 \mathrm{H}, \operatorname{Ar} H), 7.71(\mathrm{br}, 8 \mathrm{H}, \operatorname{Ar} H)$, $7.65(\mathrm{~d}, J=5.5 \mathrm{~Hz}, 4 \mathrm{H}, \operatorname{Ar} H), 7.56(\mathrm{~d}, J=5.5 \mathrm{~Hz}, 8 \mathrm{H}, \operatorname{Ar} H), 7.43(\mathrm{~d}, J=5.5 \mathrm{~Hz}, 8 \mathrm{H}, \operatorname{Ar} H), 7.40(\mathrm{~d}, J=$ $6.0 \mathrm{~Hz}, 4 \mathrm{H}, \operatorname{Ar} H), 7.26(\mathrm{~d}, J=3.5 \mathrm{~Hz}, 4 \mathrm{H}, \operatorname{Ar} H), 7.13(\mathrm{~d}, J=4.0 \mathrm{~Hz}, 4 \mathrm{H}, \operatorname{Ar} H), 7.11(\mathrm{~d}, J=3.5 \mathrm{~Hz}, 4 \mathrm{H}$, $\operatorname{Ar} H), 7.06(\mathrm{~d}, J=5.5 \mathrm{~Hz}, 4 \mathrm{H}, \operatorname{Ar} H), 6.84$ (d, J=4.0 Hz, 4H, ArH), $6.76(\mathrm{~d}, J=3.5 \mathrm{~Hz}, 4 \mathrm{H}, \operatorname{Ar} H), 6.69$ (d, $J=3.5 \mathrm{~Hz}, 4 \mathrm{H}, \operatorname{Ar} H), 5.96(\mathrm{~d}, J=3.5 \mathrm{~Hz}, 4 \mathrm{H}, \operatorname{Ar} H), 5.65(\mathrm{~d}, J=3.5 \mathrm{~Hz}, 4 \mathrm{H}, \operatorname{Ar} H), 5.32(\mathrm{~d}, J=3.0 \mathrm{~Hz}$, 4H, ArH), 5.23 (d, J=3.5 Hz, 4H, ArH), 5.00 (d, J=3.5 Hz, 4H, ArH), 4.05 (s, 12H, -OMe), 3.74 (s, $12 \mathrm{H}$, $-\mathrm{OMe}$ ), 3.67 (s, 12H, -OMe), 3.50 (s, 12H, - OMe), 3.50-3.27 (br, 40H, - $\mathrm{CH}_{2}-$ ) , 3.24 (s, 12H, - OMe), 3.24 $\left(\mathrm{s}, 12 \mathrm{H}, \mathrm{CH}_{3}\right), 3.17\left(\mathrm{~s}, 24 \mathrm{H}, \mathrm{CH}_{3}\right), 3.14\left(\mathrm{~s}, 12 \mathrm{H}, \mathrm{CH}_{3}\right), 3.01\left(\mathrm{~s}, 12 \mathrm{H}, \mathrm{CH}_{3}\right), 3.05\left(\mathrm{br}, 8 \mathrm{H},-\mathrm{CH}_{2}-\right), 2.99(\mathrm{~s}$, $\left.12 \mathrm{H}, \mathrm{CH}_{3}\right), 2.92\left(\mathrm{~s}, 24 \mathrm{H}, \mathrm{CH}_{3}\right), 2.90\left(\mathrm{~s}, 36 \mathrm{H}, \mathrm{CH}_{3}\right), 2.86\left(\mathrm{~s}, 12 \mathrm{H}, \mathrm{CH}_{3}\right), 2.65(\mathrm{~s}, 12 \mathrm{H},-\mathrm{OMe}) ;{ }^{13} \mathrm{C} \mathrm{NMR}$ (acetonitrile- $\left.d_{3} / \mathrm{D}_{2} \mathrm{O}(1: 4 \mathrm{v} / \mathrm{v}), 125 \mathrm{MHz}\right): \delta 157.2(C), 157.1(C), 156.8(C), 156.7(C), 156.0(C), 155.1$ $(C), 153.0(\mathrm{CH}), 152.7(\mathrm{CH}), 152.6(\mathrm{CH}), 151.9(\mathrm{CH}), 151.7(\mathrm{CH}), 151.1(\mathrm{CH}), 150.6(\mathrm{CH}), 150.2(C)$, $150.1(C), 150.0(C), 149.9(C), 149.6(C), 149.4(C), 148.0(C), 148.0(C), 147.6(C), 147.2(C), 147.2(C)$, $147.1(C), 140.6(C), 140.1(C), 139.9$ (C), 139.3 (C), 139.2 (C), 120.9 (CH), $120.7(C H), 120.3(C H)$, $120.2(C H), 119.9(C H), 116.4(C), 116.2(C H), 116.0(C), 115.8(C H), 115.7(C), 115.6(C H), 115.4(C H)$, $115.0(C), 114.8(C H), 114.6(C H), 114.5(C), 113.3(C H), 112.5(C H), 112.0(C H), 111.8(C H), 96.5$ $\left(\mathrm{CCl}_{4}\right), 95.7\left(\mathrm{CCl}_{4}\right), 63.4(-\mathrm{OMe}), 63.1\left(-\mathrm{CH}_{2}-\right), 62.9\left(-\mathrm{CH}_{2}-\right), 62.6\left(-\mathrm{CH}_{2}-\right), 61.1(-\mathrm{OMe}), 61.0(-\mathrm{OMe})$, $60.8(-\mathrm{OMe}), 51.5\left(\mathrm{CH}_{3}\right), 51.2\left(\mathrm{CH}_{3}\right), 51.1\left(\mathrm{CH}_{3}\right), 51.1\left(\mathrm{CH}_{3}\right), 51.0\left(\mathrm{CH}_{3}\right), 50.8\left(\mathrm{CH}_{3}\right), 50.7\left(\mathrm{CH}_{3}\right), 50.6$ $\left(\mathrm{CH}_{3}\right), 50.5\left(\mathrm{CH}_{3}\right)$. 


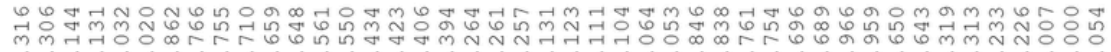

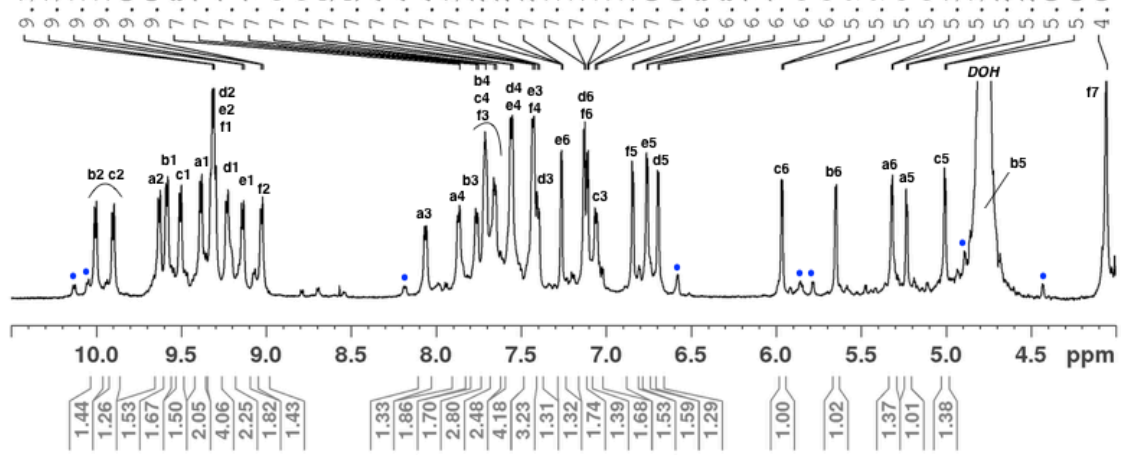

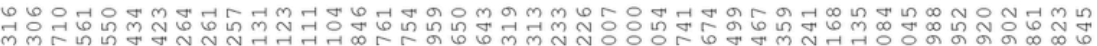

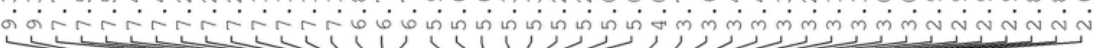
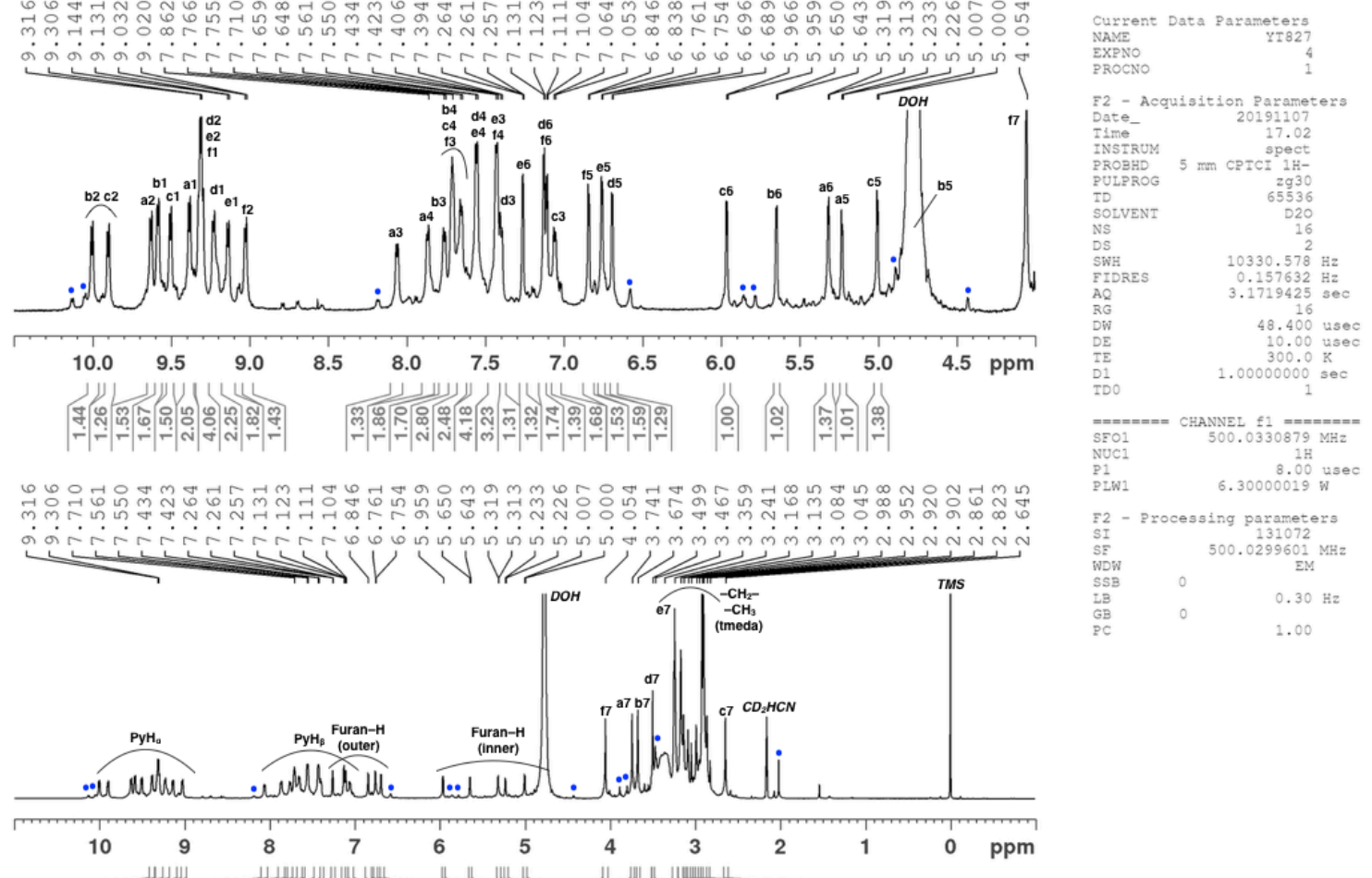

$\begin{array}{lr}\text { F2 - Processing parameters } \\ \text { SI } & 131072 \\ \text { SE } & 500.0299601 \\ \text { WDW } & \text { EM }\end{array}$ SSB
GB
PC

$0.30 \mathrm{H}=$

त.

Figure S20. ${ }^{1} \mathrm{H}$ NMR spectrum $\left(500 \mathrm{MHz}, 300 \mathrm{~K}\right.$, acetonitrile- $\left.d_{3} / \mathrm{D}_{2} \mathrm{O}(1: 4 \mathrm{v} / \mathrm{v})\right)$ of $\mathbf{1} \cdot \mathbf{C C l}_{\mathbf{4}}$.

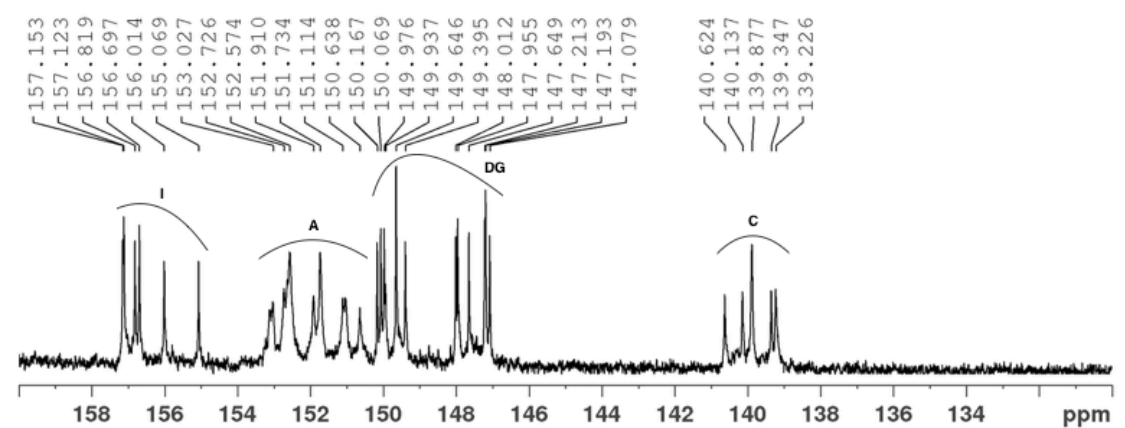

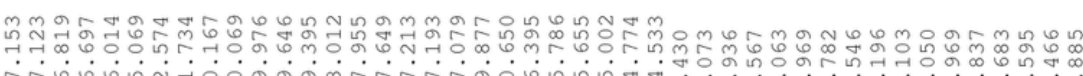

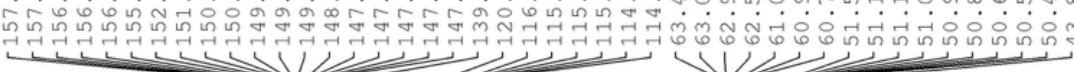
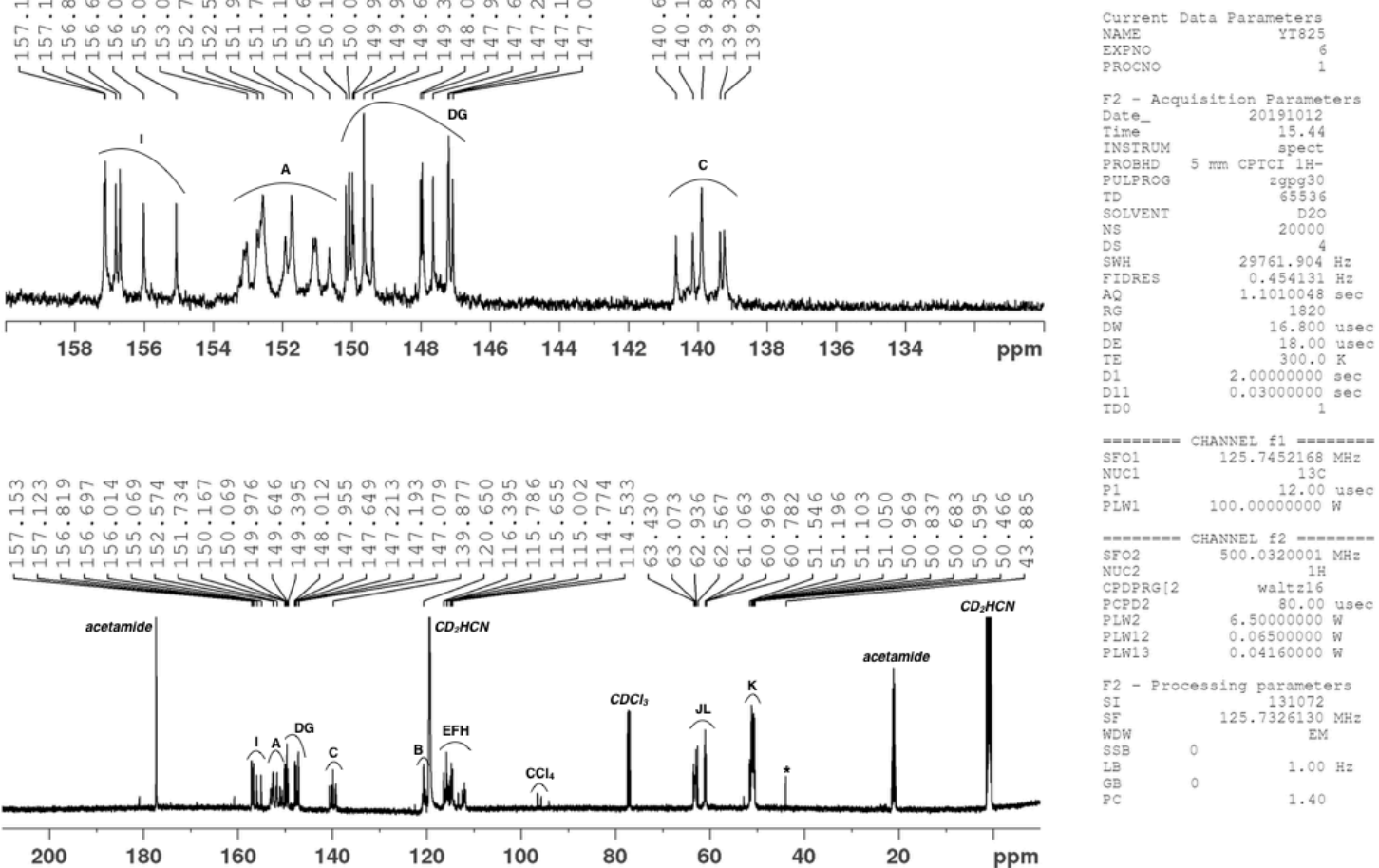

Figure S21. ${ }^{13} \mathrm{C}$ NMR spectrum $\left(125 \mathrm{MHz}, 300 \mathrm{~K}\right.$, acetonitrile- $\left.d_{3} / \mathrm{D}_{2} \mathrm{O}(1: 4 \mathrm{v} / \mathrm{v})\right)$ of $\mathbf{1} \cdot \mathbf{C C l}_{\mathbf{4}}$ 

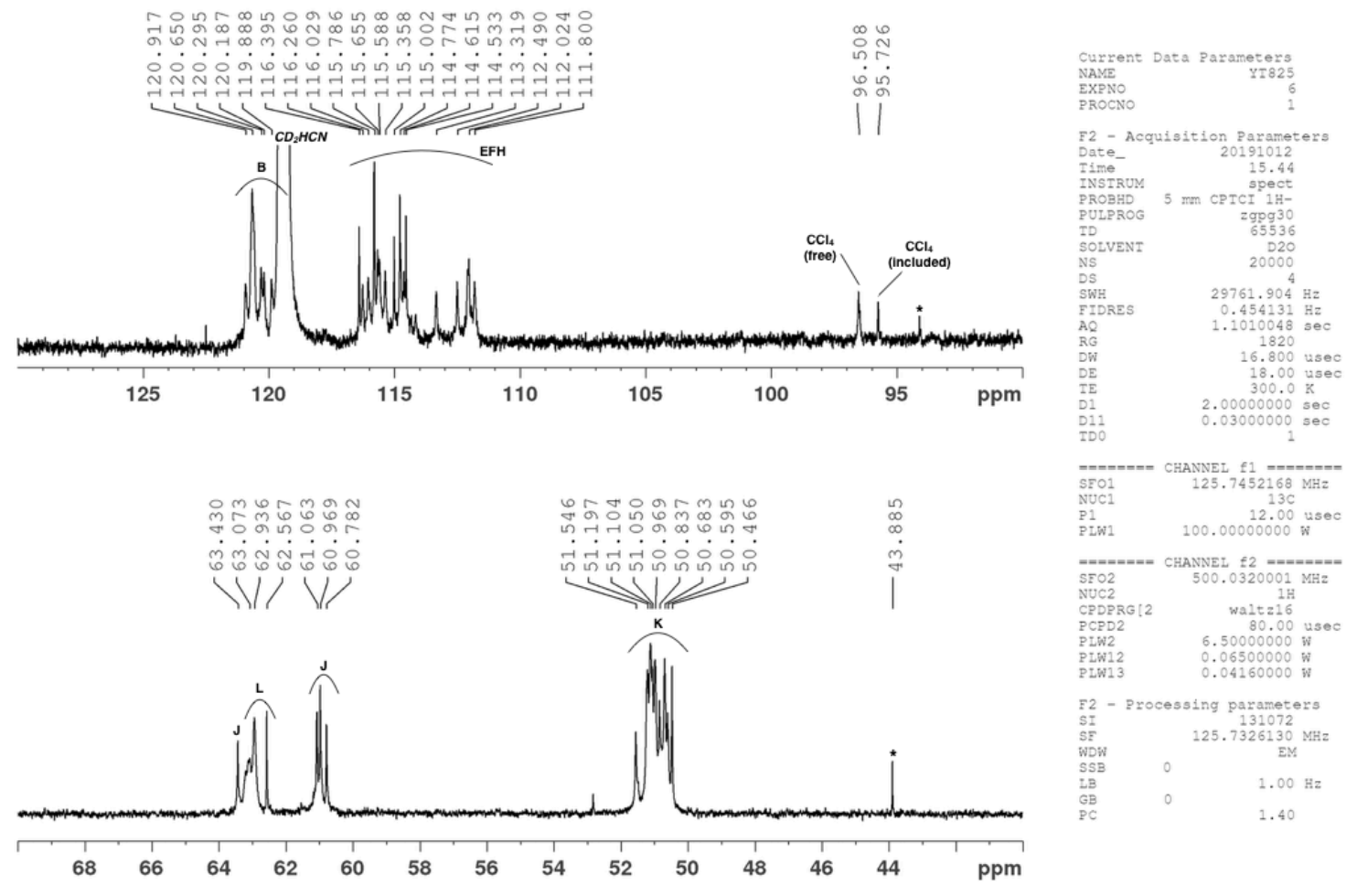

Figure S22. ${ }^{13} \mathrm{C}$ NMR spectrum $\left(125 \mathrm{MHz}, 300 \mathrm{~K}\right.$, acetonitrile- $\left.d_{3} / \mathrm{D}_{2} \mathrm{O}(1: 4 \mathrm{v} / \mathrm{v})\right)$ of $\mathbf{1} \cdot \mathbf{C C l}_{4}$.
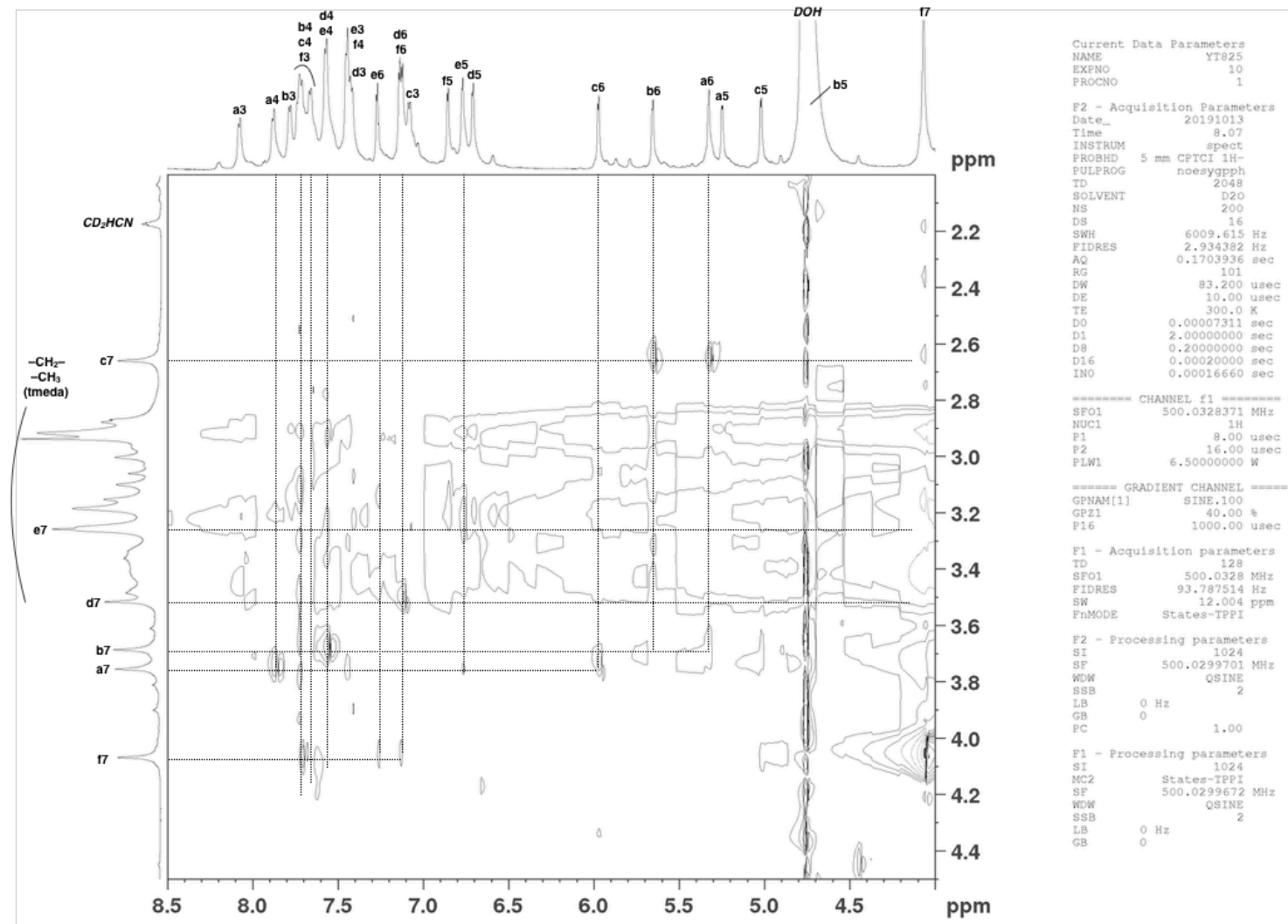

Figure S23. ${ }^{1} \mathrm{H}-{ }^{1} \mathrm{H}$ NOESY NMR spectrum $\left(500 \mathrm{MHz}, 300 \mathrm{~K}\right.$, acetonitrile- $\left.d_{3} / \mathrm{D}_{2} \mathrm{O}(1: 4 \mathrm{v} / \mathrm{v})\right)$ of $\mathbf{1} \cdot \mathbf{C C l}_{\mathbf{4}}$. 
Physical data of 1. $\mathrm{CBr}_{4}:{ }^{1} \mathrm{H}$ NMR (acetonitrile- $\left.d_{3} / \mathrm{D}_{2} \mathrm{O}(1: 4 \mathrm{v} / \mathrm{v}), 500 \mathrm{MHz}\right): \delta 9.98(\mathrm{~d}, J=6.0 \mathrm{~Hz}, 4 \mathrm{H}$, $\operatorname{Ar} H$ ), $9.87(\mathrm{~d}, J=6.0 \mathrm{~Hz}, 4 \mathrm{H}, \operatorname{Ar} H), 9.65(\mathrm{~d}, J=6.0 \mathrm{~Hz}, 4 \mathrm{H}, \operatorname{Ar} H), 9.56$ (d, $J=5.5 \mathrm{~Hz}, 4 \mathrm{H}, \operatorname{Ar} H), 9.48(\mathrm{~d}$, $J=5.5 \mathrm{~Hz}, 4 \mathrm{H}, \operatorname{Ar} H$ ), 9.39 (d, $J=5.5 \mathrm{~Hz}, 4 \mathrm{H}, \operatorname{Ar} H), 9.30$ (d, $J=6.0 \mathrm{~Hz}, 4 \mathrm{H}, \operatorname{Ar} H), 9.29$ (d, $J=6.0 \mathrm{~Hz}$, 4H, $\operatorname{Ar} H), 9.26(\mathrm{~d}, J=6.0 \mathrm{~Hz}, 4 \mathrm{H}, \operatorname{Ar} H), 9.24(\mathrm{~d}, J=6.0 \mathrm{~Hz}, 4 \mathrm{H}, \operatorname{Ar} H), 9.11(\mathrm{~d}, J=6.0 \mathrm{~Hz}, 4 \mathrm{H}, \operatorname{Ar} H), 9.02$ (d, $J=6.0 \mathrm{~Hz}, 4 \mathrm{H}, \operatorname{Ar} H), 8.05(\mathrm{~d}, J=5.0 \mathrm{~Hz}, 4 \mathrm{H}, \operatorname{Ar} H), 7.90$ (d, $J=6.0 \mathrm{~Hz}, 4 \mathrm{H}, \operatorname{Ar} H), 7.77$ (d, $J=5.0 \mathrm{~Hz}$, 4H, $\operatorname{Ar} H$ ), $7.72(\mathrm{~d}, J=6.0 \mathrm{~Hz}, 4 \mathrm{H}, \operatorname{Ar} H), 7.72(\mathrm{~d}, J=6.0 \mathrm{~Hz}, 4 \mathrm{H}, \operatorname{Ar} H), 7.71(\mathrm{~d}, J=6.0 \mathrm{~Hz}, 4 \mathrm{H}, \operatorname{Ar} H), 7.53$ $(\mathrm{d}, J=5.0 \mathrm{~Hz}, 8 \mathrm{H}, \operatorname{Ar} H), 7.43(\mathrm{~d}, J=4.5 \mathrm{~Hz}, 8 \mathrm{H}, \operatorname{Ar} H), 7.36(\mathrm{~d}, J=5.5 \mathrm{~Hz}, 4 \mathrm{H}, \operatorname{Ar} H), 7.28$ (d, $J=3.0 \mathrm{~Hz}$, 4H, $\operatorname{Ar} H), 7.15(\mathrm{~d}, J=3.5 \mathrm{~Hz}, 8 \mathrm{H}, \operatorname{Ar} H), 7.06(\mathrm{~d}, J=5.5 \mathrm{~Hz}, 4 \mathrm{H}, \operatorname{Ar} H), 6.85(\mathrm{~d}, J=3.5 \mathrm{~Hz}, 4 \mathrm{H}, \operatorname{Ar} H), 6.79$ $(\mathrm{d}, J=3.0 \mathrm{~Hz}, 4 \mathrm{H}, \operatorname{Ar} H), 6.69(\mathrm{~d}, J=3.5 \mathrm{~Hz}, 4 \mathrm{H}, \operatorname{Ar} H), 5.99(\mathrm{~d}, J=3.0 \mathrm{~Hz}, 4 \mathrm{H}, \operatorname{Ar} H), 5.65(\mathrm{~d}, J=3.0 \mathrm{~Hz}$, $4 \mathrm{H}, \operatorname{Ar} H$ ), 5.26 (s, 8H, $\operatorname{Ar} H$ ), 5.01 (d, J=3.5 Hz, 4H, $\operatorname{Ar} H$ ), 4.10 (s, 12H, -OMe), 3.80 (s, 12H, -OMe), 3.62 (s, $12 \mathrm{H},-\mathrm{OMe}$ ), 3.50 (s, 12H, $-\mathrm{OMe}$ ), 3.48-3.27 (br, 40H, $-\mathrm{CH}_{2}-$ ), 3.24 (s, 12H, $-\mathrm{OMe}$ ), $3.23(\mathrm{~s}, 12 \mathrm{H}$, $\left.\mathrm{CH}_{3}\right), 3.17\left(\mathrm{~s}, 24 \mathrm{H}, \mathrm{CH}_{3}\right), 3.14\left(\mathrm{~s}, 12 \mathrm{H}, \mathrm{CH}_{3}\right), 3.09$ (s, $\left.12 \mathrm{H}, \mathrm{CH}_{3}\right), 3.04$ (br, $\left.8 \mathrm{H},-\mathrm{CH}_{2}-\right), 2.97\left(\mathrm{~s}, 12 \mathrm{H}, \mathrm{CH}_{3}\right.$ ), 2.92 (s, 24H, $\left.\mathrm{CH}_{3}\right), 2.91\left(\mathrm{~s}, 12 \mathrm{H}, \mathrm{CH}_{3}\right), 2.89$ (s, $\left.24 \mathrm{H}, \mathrm{CH}_{3}\right), 2.85\left(\mathrm{~s}, 12 \mathrm{H}, \mathrm{CH}_{3}\right), 2.83(\mathrm{~s}, 12 \mathrm{H},-\mathrm{OMe}) ;{ }^{13} \mathrm{C}$ NMR (acetonitrile- $\left.d_{3} / \mathrm{D}_{2} \mathrm{O}(1: 4 \mathrm{v} / \mathrm{v}), 125 \mathrm{MHz}\right): \delta 157.1(C), 156.8(C), 156.7$ (C), $156.1(C), 154.8(C)$, 153.0 (CH), $152.8(\mathrm{CH}), 152.6(\mathrm{CH}), 152.5(\mathrm{CH}), 151.9(\mathrm{CH}), 151.7(\mathrm{CH}), 151.1(\mathrm{CH}), 150.2(\mathrm{C}), 150.1$ (C), $150.0(C), 149.9(C), 147.9$ (C), 147.7 (C), $147.2(C), 147.1$ (C), 146.9 (C), $140.6(C), 140.1(C), 140.0$ (C), $139.7(C), 139.4(C), 139.3(C), 120.7(C H), 120.5(C H), 120.4(C H), 120.4(C H), 120.2(C H), 119.8$ $(\mathrm{CH}), 116.5(C), 116.2(\mathrm{CH}), 116.0(\mathrm{CH}), 115.8(\mathrm{CH}), 115.7(\mathrm{C}), 115.5(\mathrm{CH}), 115.3(\mathrm{C}), 115.1(\mathrm{C}), 114.8$ $(\mathrm{CH}), 114.6(\mathrm{CH}), 113.3(\mathrm{CH}), 112.4(\mathrm{C}), 112.0(\mathrm{CH}), 111.8(\mathrm{C}), 63.4(-\mathrm{OMe}), 63.2(-\mathrm{OMe}), 63.1(-\mathrm{OMe})$, $62.9(-\mathrm{OMe}), 62.6$ (-OMe), $61.2(-\mathrm{OMe}), 61.1(-\mathrm{OMe}), 61.0(-\mathrm{OMe}), 61.0(-\mathrm{OMe}), 60.9$ (-OMe), $60.8(-$ $\mathrm{OMe}), 51.5\left(\mathrm{CH}_{3}\right), 51.2\left(\mathrm{CH}_{3}\right), 51.1\left(\mathrm{CH}_{3}\right), 51.0\left(\mathrm{CH}_{3}\right), 50.9\left(\mathrm{CH}_{3}\right), 50.9\left(\mathrm{CH}_{3}\right), 50.7\left(\mathrm{CH}_{3}\right), 50.6\left(\mathrm{CH}_{3}\right)$, $50.5\left(\mathrm{CH}_{3}\right),-27.5\left(\mathrm{CBr}_{4}\right),-32.5\left(\mathrm{CBr}_{4}\right)$. 

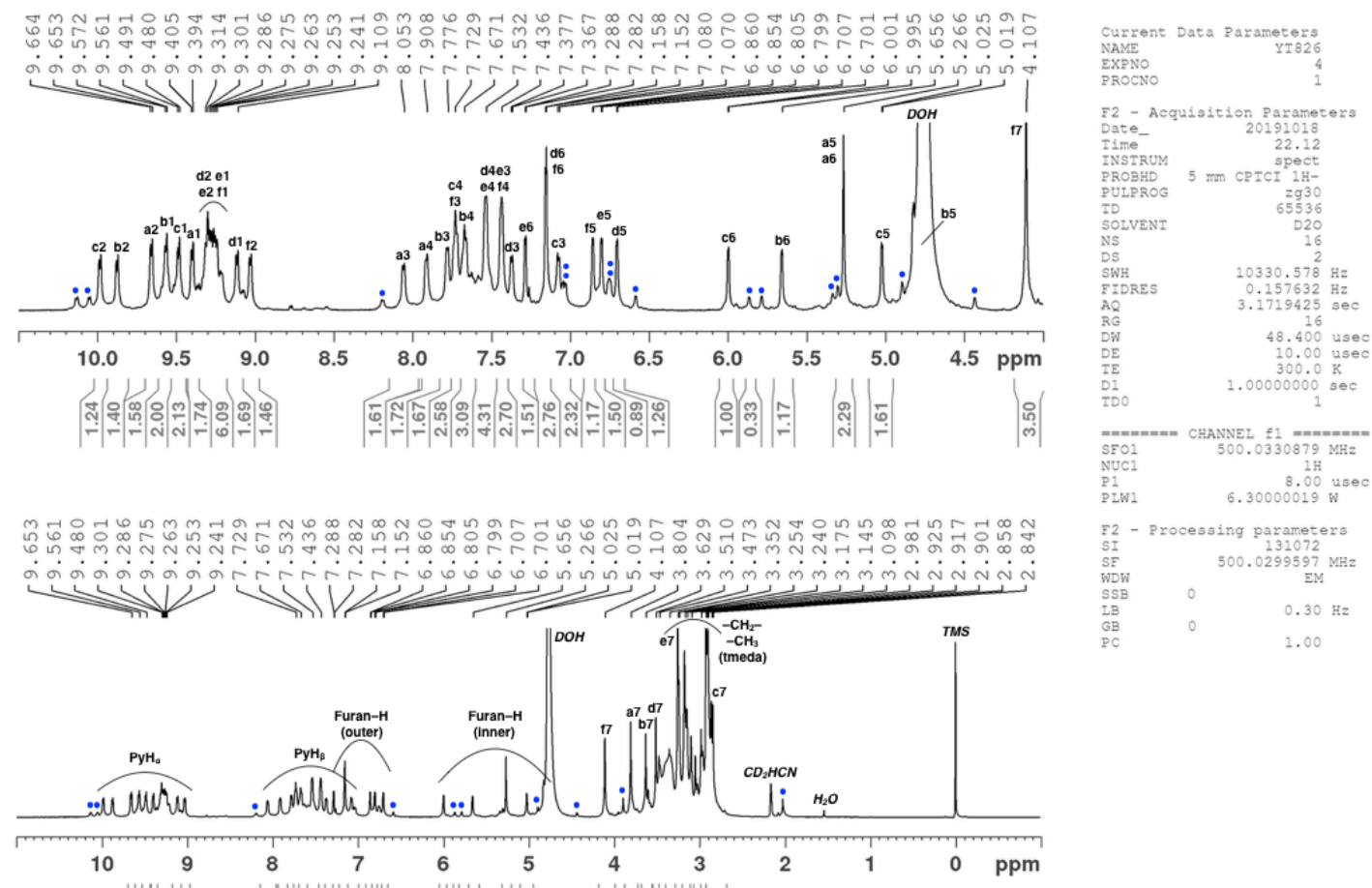

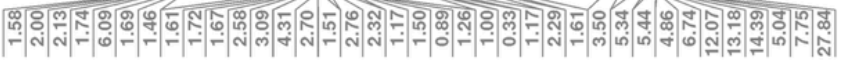

Figure S24. ${ }^{1} \mathrm{H}$ NMR spectrum $\left(500 \mathrm{MHz}, 300 \mathrm{~K}\right.$, acetonitrile- $\left.d_{3} / \mathrm{D}_{2} \mathrm{O}(1: 4 \mathrm{v} / \mathrm{v})\right)$ of $\mathbf{1} \cdot \mathbf{C B r} \mathbf{B r}_{\mathbf{4}}$ (signals from empty cage $\mathbf{1}$ are denoted by blue dots).
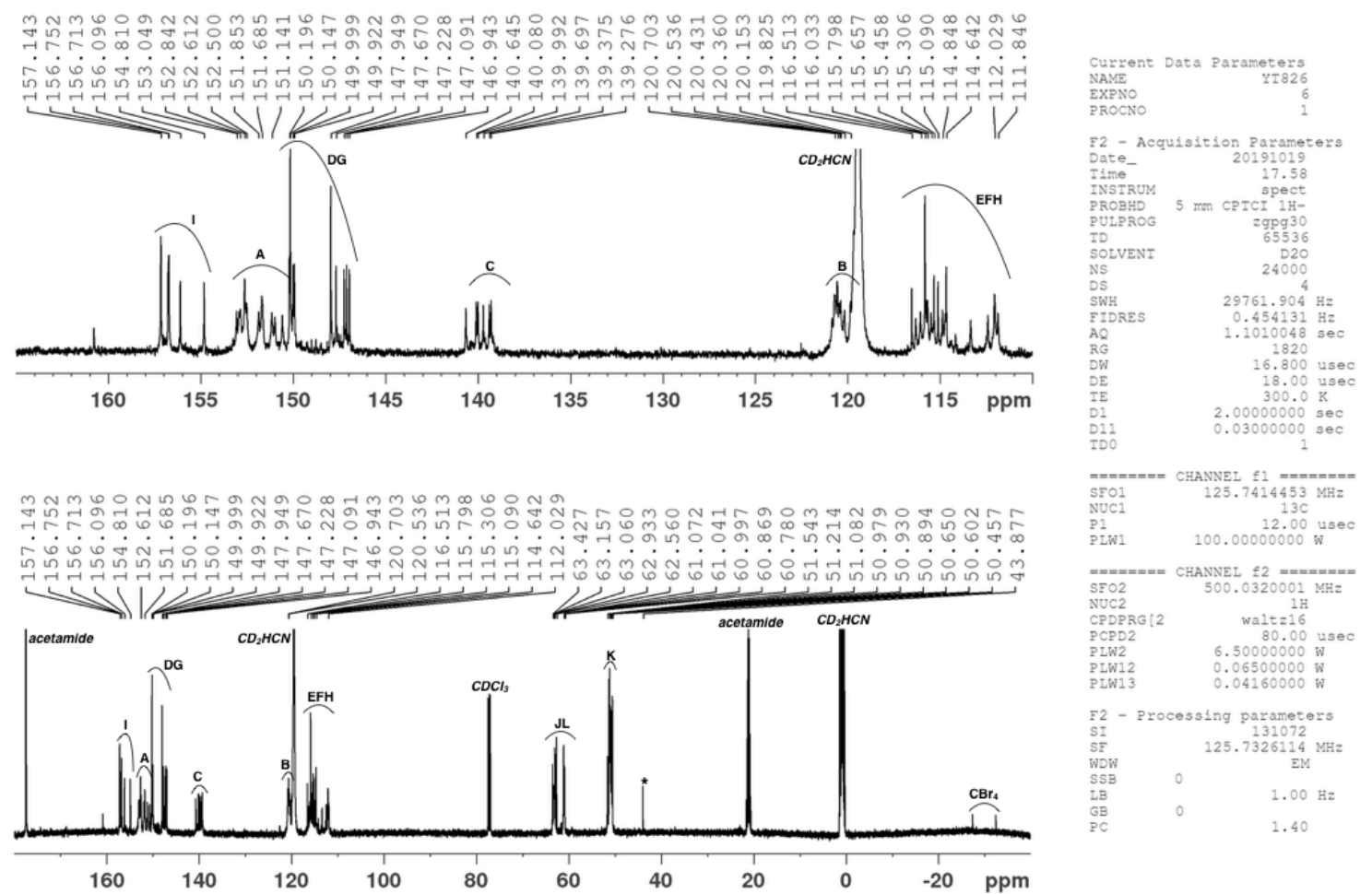

Figure 25. ${ }^{13} \mathrm{C}$ NMR spectrum $\left(125 \mathrm{MHz}, 300 \mathrm{~K}\right.$, acetonitrile- $\left.d_{3} / \mathrm{D}_{2} \mathrm{O}(1: 4 \mathrm{v} / \mathrm{v})\right)$ of $\mathbf{1} \cdot \mathbf{C B r}_{\mathbf{4}}$ (a signal from impurity is denoted by an asterisk). 

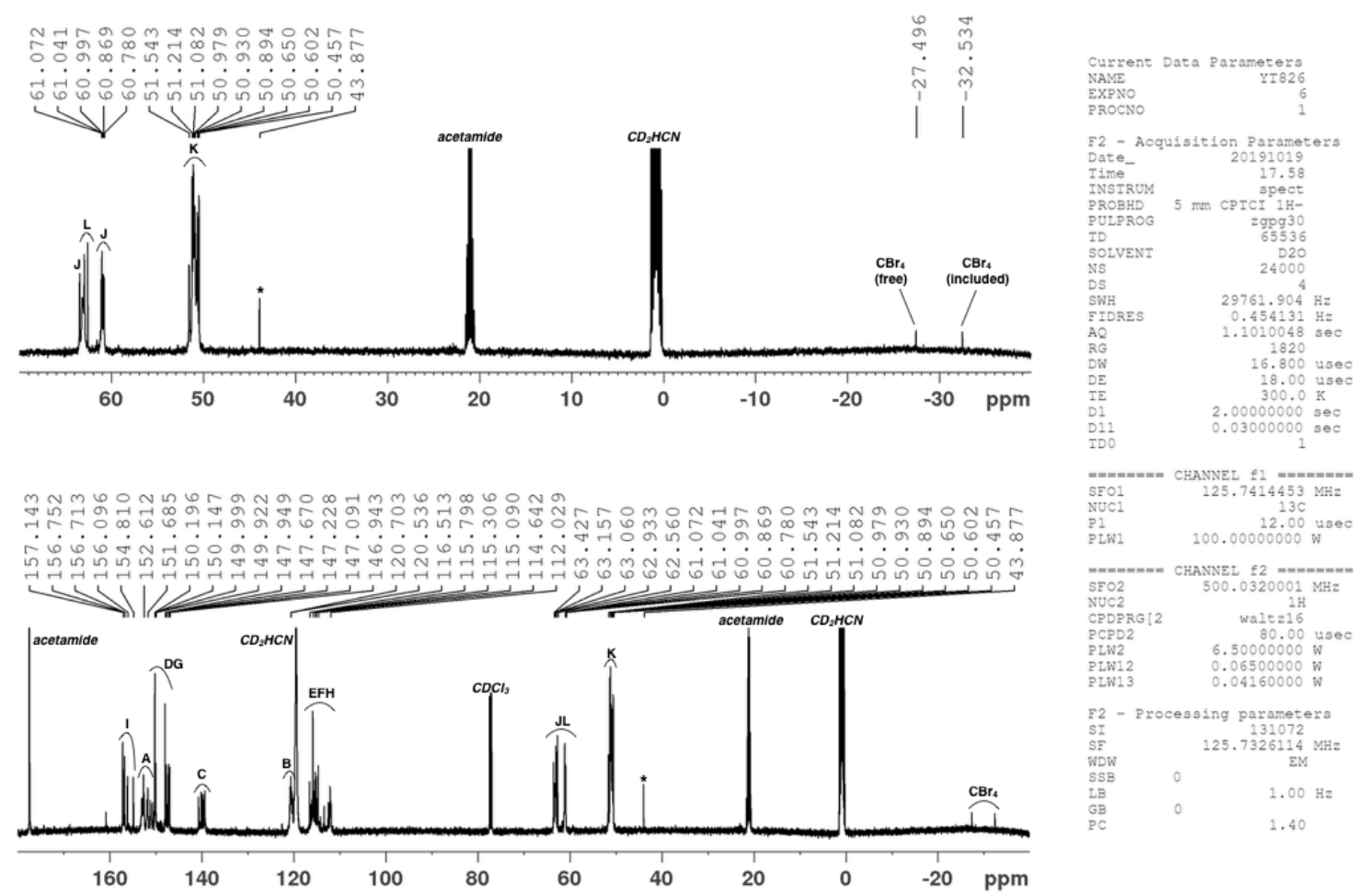

Figure S26. ${ }^{13} \mathrm{C}$ NMR spectrum $\left(125 \mathrm{MHz}, 300 \mathrm{~K}\right.$, acetonitrile- $\left.d_{3} / \mathrm{D}_{2} \mathrm{O}(1: 4 \mathrm{v} / \mathrm{v})\right)$ of $\mathbf{1} \cdot \mathbf{C B r}_{\mathbf{4}}$ (a signal from impurity is denoted by an asterisk).

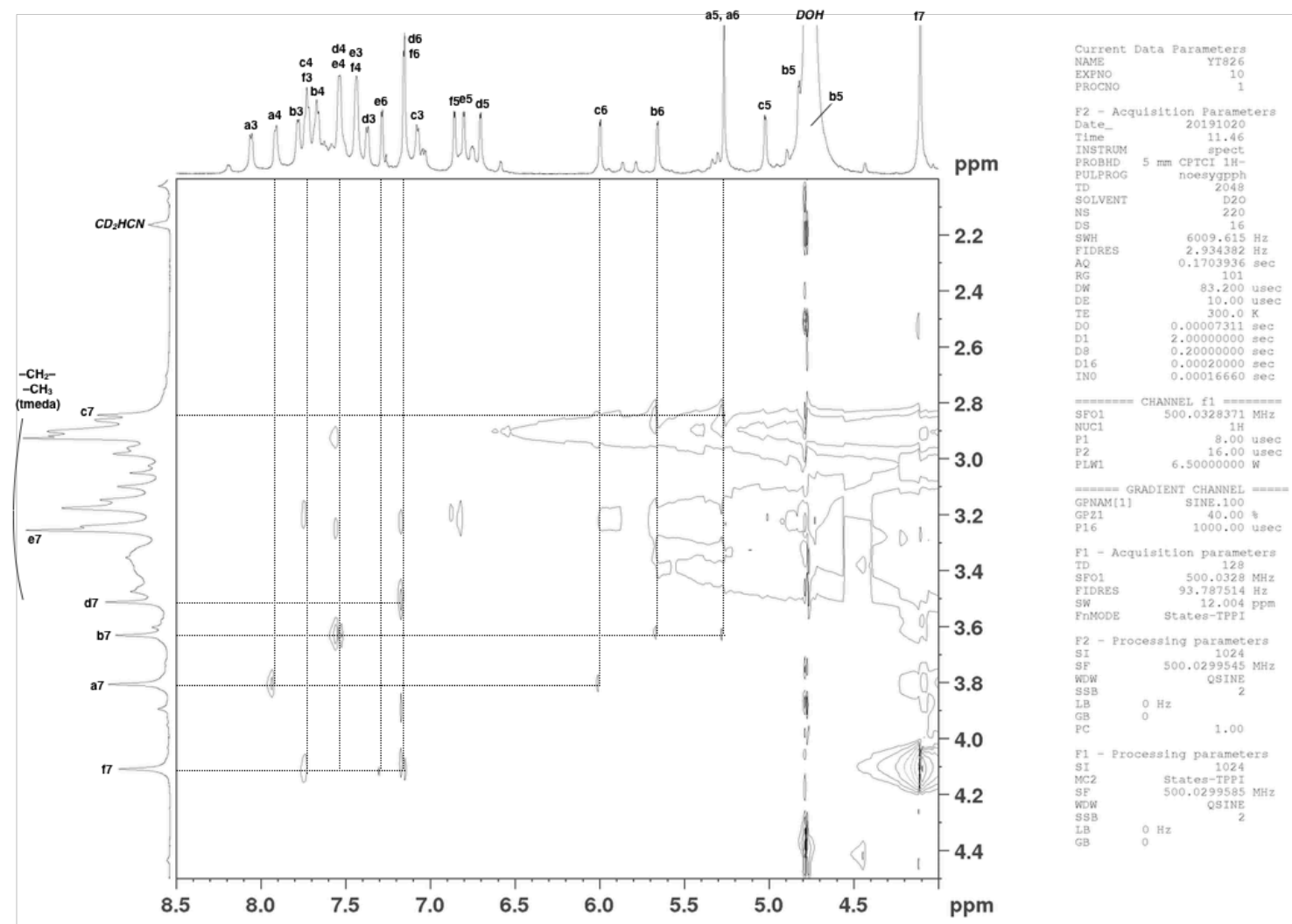

Figure S27. ${ }^{1} \mathrm{H}-{ }^{1} \mathrm{H}$ NOESY NMR spectrum $\left(500 \mathrm{MHz}, 300 \mathrm{~K}\right.$, acetonitrile- $\left.d_{3} / \mathrm{D}_{2} \mathrm{O}(1: 4 \mathrm{v} / \mathrm{v})\right)$ of $\mathbf{1} \cdot \mathbf{C B r}_{\mathbf{4}}$. 
Physical data of 1•CHBr ${ }_{3}:{ }^{1} \mathrm{H}$ NMR (acetonitrile- $\left.d_{3} / \mathrm{D}_{2} \mathrm{O}(1: 4 \mathrm{v} / \mathrm{v}), 500 \mathrm{MHz}\right): \delta 10.05$ (d, $J=5.0 \mathrm{~Hz}, 4 \mathrm{H}$, $\operatorname{Ar} H$ ), 9.97 (d, $J=5.0 \mathrm{~Hz}, 4 \mathrm{H}, \operatorname{Ar} H), 9.63(\mathrm{~d}, J=6.0 \mathrm{~Hz}, 4 \mathrm{H}, \operatorname{Ar} H), 9.61$ (d, $J=5.0 \mathrm{~Hz}, 4 \mathrm{H}, \operatorname{Ar} H), 9.52(\mathrm{~d}$, $J=5.5 \mathrm{~Hz}, 4 \mathrm{H}, \operatorname{Ar} H), 9.41(\mathrm{~d}, J=6.0 \mathrm{~Hz}, 4 \mathrm{H}, \operatorname{Ar} H), 9.39$ (d, $J=5.5 \mathrm{~Hz}, 4 \mathrm{H}, \operatorname{Ar} H), 9.38$ (d, $J=5.0 \mathrm{~Hz}$, 4H, $\operatorname{Ar} H), 9.30(\mathrm{~d}, J=5.5 \mathrm{~Hz}, 4 \mathrm{H}, \operatorname{Ar} H), 9.26(\mathrm{~d}, J=6.0 \mathrm{~Hz}, 4 \mathrm{H}, \operatorname{Ar} H), 9.16(\mathrm{~d}, J=5.5 \mathrm{~Hz}, 4 \mathrm{H}, \operatorname{Ar} H), 9.08$ (d, $J=5.0 \mathrm{~Hz}, 4 \mathrm{H}, \operatorname{Ar} H), 8.06(\mathrm{~d}, J=5.0 \mathrm{~Hz}, 4 \mathrm{H}, \operatorname{Ar} H), 7.86(\mathrm{~d}, J=5.5 \mathrm{~Hz}, 4 \mathrm{H}, \operatorname{Ar} H), 7.78(\mathrm{~d}, J=4.5 \mathrm{~Hz}$, 4H, $\operatorname{Ar} H), 7.71(\mathrm{~d}, J=5.5 \mathrm{~Hz}, 4 \mathrm{H}, \operatorname{Ar} H), 7.69(\mathrm{~d}, J=5.0 \mathrm{~Hz}, 4 \mathrm{H}, \operatorname{Ar} H), 7.63$ (d, $J=5.5 \mathrm{~Hz}, 4 \mathrm{H}, \operatorname{Ar} H), 7.59$ (br, 8H, ArH), 7.51 (d, $J=5.5 \mathrm{~Hz}, 4 \mathrm{H}, \operatorname{Ar} H), 7.45(\mathrm{~d}, J=6.0 \mathrm{~Hz}, 4 \mathrm{H}, \operatorname{Ar} H), 7.43(\mathrm{~d}, J=6.0 \mathrm{~Hz}, 4 \mathrm{H}, \operatorname{Ar} H)$, 7.21 (br, 4H, ArH), 7.11 (br, 12H, ArH), $6.82(\mathrm{~d}, J=3.0 \mathrm{~Hz}, 4 \mathrm{H}, \operatorname{Ar} H), 6.74$ (d, $J=3.0 \mathrm{~Hz}, 4 \mathrm{H}, \operatorname{Ar} H), 6.73$ (d, $J=3.0 \mathrm{~Hz}, 4 \mathrm{H}, \operatorname{Ar} H$ ), 5.94 (br, 4H, $\operatorname{Ar} H$ ), 5.72 (br, 4H, $\operatorname{Ar} H$ ), 5.36 (br, 4H, $\operatorname{Ar} H$ ), 5.21 (br, 4H, $\operatorname{Ar} H$ ), 4.98 (br, 4H, $\mathrm{Ar} H$ ), 4.07 (s, 12H, -OMe), 3.72 (s, 12H, -OMe), 3.71 (s, 12H, -OMe), 3.50 (s, 12H, -OMe), 3.48-3.30 (br, 40H, $-\mathrm{CH}_{2}-$ ), 3.27 (s, $\left.12 \mathrm{H},-\mathrm{OMe}\right), 3.23\left(\mathrm{~s}, 12 \mathrm{H}, \mathrm{CH}_{3}\right), 3.16\left(\mathrm{~s}, 24 \mathrm{H}, \mathrm{CH}_{3}\right), 3.14(\mathrm{~s}, 12 \mathrm{H}$, $\mathrm{CH}_{3}$ ), 3.07 (s, $12 \mathrm{H}, \mathrm{CH}_{3}$ ), 3.04 (s, $12 \mathrm{H}, \mathrm{CH}_{3}$ ), 2.99 (br, 8H, $-\mathrm{CH}_{2}-$ ), $2.92\left(\mathrm{~s}, 24 \mathrm{H}, \mathrm{CH}_{3}\right), 2.91\left(\mathrm{~s}, 24 \mathrm{H}, \mathrm{CH}_{3}\right)$, $2.88\left(\mathrm{~s}, 24 \mathrm{H}, \mathrm{CH}_{3}\right), 2.58(\mathrm{~s}, 12 \mathrm{H},-\mathrm{OMe})$.

Physical data of $\mathbf{1} \cdot \mathbf{C H}_{2} \mathbf{I}_{\mathbf{2}}:{ }^{1} \mathrm{H}$ NMR (acetonitrile- $\left.d_{3} / \mathrm{D}_{2} \mathrm{O}(1: 4 \mathrm{v} / \mathrm{v}), 500 \mathrm{MHz}\right): \delta 9.98(\mathrm{~d}, J=5.5 \mathrm{~Hz}, 4 \mathrm{H}$, $\operatorname{Ar} H), 9.91(\mathrm{~d}, J=5.5 \mathrm{~Hz}, 4 \mathrm{H}, \operatorname{Ar} H), 9.59(\mathrm{~d}, J=5.5 \mathrm{~Hz}, 8 \mathrm{H}, \operatorname{Ar} H), 9.54$ (d, $J=5.5 \mathrm{~Hz}, 4 \mathrm{H}, \operatorname{Ar} H), 9.52(\mathrm{~d}$, $J=5.0 \mathrm{~Hz}, 4 \mathrm{H}, \operatorname{Ar} H), 9.35$ (br, 4H, $\operatorname{Ar} H), 9.33$ (d, $J=6.0 \mathrm{~Hz}, 4 \mathrm{H}, \operatorname{Ar} H), 9.26(\mathrm{~d}, J=6.0 \mathrm{~Hz}, 4 \mathrm{H}, \operatorname{Ar} H$ ), $9.23(\mathrm{~d}, J=5.5 \mathrm{~Hz}, 4 \mathrm{H}, \operatorname{Ar} H), 9.12(\mathrm{~d}, J=6.0 \mathrm{~Hz}, 4 \mathrm{H}, \operatorname{Ar} H), 9.02$ (d, $J=5.5 \mathrm{~Hz}, 4 \mathrm{H}, \operatorname{Ar} H), 8.03$ (d, $J=$ $5.0 \mathrm{~Hz}, 4 \mathrm{H}, \operatorname{Ar} H), 7.83(\mathrm{~d}, J=5.5 \mathrm{~Hz}, 4 \mathrm{H}, \operatorname{Ar} H), 7.76(\mathrm{~d}, J=6.0 \mathrm{~Hz}, 4 \mathrm{H}, \operatorname{Ar} H), 7.34$ (br, 4H, ArH), 7.64 (br, 8H, ArH), 7.56 (br, 12H, ArH), 7.40 (br, 8H, ArH), 7.16 (br, 4H, ArH), 7.08 (br, 12H, ArH), 6.78 (br, 4H, ArH), 6.73 (br, 4H, ArH), 6.69 (br, 4H, ArH), 5.88 (br, 4H, ArH), 5.73 (br, 4H, ArH), 5.34 (br, 4H, $\operatorname{Ar} H$ ), 5.17 (br, 4H, ArH), 4.24 (br, 4H, ArH), 4.05 (br, 4H, ArH), 3.98 (s, 12H, -OMe), 3.72 (s, 12H, $\mathrm{OMe}$ ), 3.69 (s, 12H, -OMe), 3.41-3.26 (br, 40H, $-\mathrm{CH}_{2}-$ ), 3.22 (s, $12 \mathrm{H}, \mathrm{CH}_{3}$ ), 3.20 (s, $\left.12 \mathrm{H}, \mathrm{CH}_{3}\right), 3.13$ (s, 24H, $\mathrm{CH}_{3}$ ), 3.04 (s, $\left.12 \mathrm{H}, \mathrm{CH}_{3}\right), 2.99$ (br, 8H, $\left.-\mathrm{CH}_{2}-\right), 2.97\left(\mathrm{~s}, 12 \mathrm{H}, \mathrm{CH}_{3}\right), 2.93\left(\mathrm{~s}, 12 \mathrm{H}, \mathrm{CH}_{3}\right), 2.89(\mathrm{~s}, 36 \mathrm{H}$, $\left.\mathrm{CH}_{3}\right), 2.85\left(\mathrm{~s}, 24 \mathrm{H}, \mathrm{CH}_{3}\right), 2.46(\mathrm{~s}, 12 \mathrm{H},-\mathrm{OMe})$.

Inclusion of $\mathrm{CHCl}_{3},\left(\mathrm{CHCl}_{2}\right)_{2}$, and trimethylsilyl acetylene (TMSA) were carried out with $1.25 \mathrm{mM}$ solution of $\mathbf{1}$ in acetonitrile- $d_{3} / \mathrm{D}_{2} \mathrm{O}(800 \mu \mathrm{L}, 1: 4 \mathrm{v} / \mathrm{v})$.

Physical data of $1 \cdot \mathbf{C H C l}_{3}:{ }^{1} \mathrm{H}$ NMR (acetonitrile- $\left.d_{3} / \mathrm{D}_{2} \mathrm{O}(1: 4 \mathrm{v} / \mathrm{v}), 500 \mathrm{MHz}\right): \delta 10.14(\mathrm{br}, 4 \mathrm{H}, \mathrm{Ar} H$ ), 10.07 (br, 4H, $\operatorname{Ar} H$ ), 9.65 (d, $J=5.0 \mathrm{~Hz}, 8 \mathrm{H}, \operatorname{Ar} H$ ), 9.56 (d, $J=5.0 \mathrm{~Hz}, 4 \mathrm{H}, \operatorname{Ar} H$ ), 9.50 (br, 4H, ArH), 9.46 (br, 4H, ArH), 9.40 (d, $J=5.0 \mathrm{~Hz}, 4 \mathrm{H}, \operatorname{Ar} H), 9.35$ (d, $J=5.0 \mathrm{~Hz}, 4 \mathrm{H}, \operatorname{Ar} H), 9.27$ (d, $J=5.0 \mathrm{~Hz}, 4 \mathrm{H}, \operatorname{Ar} H$ ), 9.22 (br, 4H, ArH), 9.12 (d, J=5.5 Hz, 4H, ArH), 8.14 (br, 4H, ArH), 7.74 (br, 8H, ArH), 7.69 (br, 12H, $\operatorname{Ar} H$ ), 7.64 (br, 8H, $\operatorname{Ar} H$ ), 7.55 (br, 4H, $\operatorname{Ar} H$ ), 7.50 (br, 4H, $\operatorname{Ar} H$ ), 7.46 (br, 4H, $\operatorname{Ar} H$ ), 7.29 (br, 4H, $\operatorname{Ar} H$ ), 
7.20 (br, 4H, ArH), 7.14 (d, $J=6.5 \mathrm{~Hz}, 4 \mathrm{H}, \operatorname{Ar} H), 7.10$ (br, 4H, ArH), 6.97 (br, 4H, ArH), 5.93 (br, 4H, $\operatorname{Ar} H$ ), 5.75 (br, 4H, $\operatorname{Ar} H$ ), 5.37 (br, 4H, $\operatorname{Ar} H$ ), 5.28 (br, 4H, $\operatorname{Ar} H$ ), 4.99 (br, 4H, $\operatorname{Ar} H$ ), 4.42 (br, 4H, $\operatorname{Ar} H$ ), 3.99 (s, 12H, -OMe), 3.77 (s, 12H, -OMe), 3.68 (s, 12H, -OMe), 3.47-3.32 (br, 40H, $-\mathrm{CH}_{2}-$ ), $3.30(\mathrm{~s}, 12 \mathrm{H}$, $-\mathrm{OMe}$ ), 3.28 (s, 12H, $\mathrm{CH}_{3}$ ), 3.27 (s, $12 \mathrm{H}, \mathrm{CH}_{3}$ ), 3.19 (br, 24H, $\mathrm{CH}_{3}$ ), 3.03 (s, $\left.12 \mathrm{H}, \mathrm{CH}_{3}\right), 2.99$ (br, 8H, $\left.\mathrm{CH}_{2}-\right), 2.83$ (s, 36H, $\left.\mathrm{CH}_{3}\right), 2.47$ (br, $12 \mathrm{H},-\mathrm{OMe}$ ).

Physical data of 1•( $\left.\mathbf{C H C l}_{2}\right)_{2}:{ }^{1} \mathrm{H}$ NMR (acetonitrile- $\left.d_{3} / \mathrm{D}_{2} \mathrm{O}(1: 4 \mathrm{v} / \mathrm{v}), 500 \mathrm{MHz}\right): \delta 10.02(\mathrm{~d}, J=6.0 \mathrm{~Hz}$, 4H, $\operatorname{Ar} H), 9.94(\mathrm{~d}, J=6.0 \mathrm{~Hz}, 4 \mathrm{H}, \operatorname{Ar} H), 9.65(\mathrm{~d}, J=6.0 \mathrm{~Hz}, 8 \mathrm{H}, \operatorname{Ar} H), 9.59(\mathrm{~d}, J=6.0 \mathrm{~Hz}, 8 \mathrm{H}, \operatorname{Ar} H), 9.51$ $(\mathrm{d}, J=6.5 \mathrm{~Hz}, 4 \mathrm{H}, \operatorname{Ar} H), 9.40(\mathrm{~d}, J=6.0 \mathrm{~Hz}, 4 \mathrm{H}, \operatorname{Ar} H), 9.30(\mathrm{~d}, J=5.5 \mathrm{~Hz}, 4 \mathrm{H}, \operatorname{Ar} H), 9.27$ (d, $J=5.5 \mathrm{~Hz}$, 4H, ArH), 9.14 (d, $J=6.0 \mathrm{~Hz}, 4 \mathrm{H}, \operatorname{Ar} H$ ), 9.08 (d, $J=5.5 \mathrm{~Hz}, 4 \mathrm{H}, \operatorname{Ar} H$ ), 8.05 (d, $J=5.0 \mathrm{~Hz}, 4 \mathrm{H}, \operatorname{Ar} H$ ), 7.89 (d, $J=5.5 \mathrm{~Hz}, 4 \mathrm{H}, \operatorname{Ar} H$ ), 7.80 (d, $J=6.5 \mathrm{~Hz}, 4 \mathrm{H}, \operatorname{Ar} H$ ), 7.71 (d, $J=6.0 \mathrm{~Hz}, 8 \mathrm{H}, \operatorname{Ar} H$ ), $7.56(\mathrm{br}, 12 \mathrm{H}, \operatorname{Ar} H$ ), $7.50(\mathrm{~d}, J=5.0 \mathrm{~Hz}, 4 \mathrm{H}, \operatorname{Ar} H), 7.45(\mathrm{~d}, J=6.0 \mathrm{~Hz}, 4 \mathrm{H}, \operatorname{Ar} H), 7.40(\mathrm{~d}, J=5.5 \mathrm{~Hz}, 4 \mathrm{H}, \operatorname{Ar} H), 7.22(\mathrm{~d}, J=$ $3.5 \mathrm{~Hz}, 4 \mathrm{H}, \operatorname{Ar} H$ ), 7.13 (d, $J=3.0 \mathrm{~Hz}, 12 \mathrm{H}, \operatorname{Ar} H), 6.82$ (d, $J=3.5 \mathrm{~Hz}, 4 \mathrm{H}, \operatorname{Ar} H$ ), 6.75 (d, $J=3.5 \mathrm{~Hz}, 4 \mathrm{H}$, $\operatorname{Ar} H$ ), 6.74 (d, $J=3.5 \mathrm{~Hz}, 4 \mathrm{H}, \operatorname{Ar} H), 6.01(\mathrm{~d}, J=4.0 \mathrm{~Hz}, 4 \mathrm{H}, \operatorname{Ar} H), 5.69$ (d, $J=4.0 \mathrm{~Hz}, 4 \mathrm{H}, \operatorname{Ar} H), 5.33$ (d, $J=3.5 \mathrm{~Hz}, 4 \mathrm{H}, \operatorname{Ar} H), 5.23$ (d, $J=3.5 \mathrm{~Hz}, 4 \mathrm{H}, \operatorname{Ar} H), 5.03$ (d, $J=3.5 \mathrm{~Hz}, 4 \mathrm{H}, \operatorname{Ar} H), 4.03$ (s, 12H, -OMe), 3.74 (s, $12 \mathrm{H},-\mathrm{OMe}), 3.70$ (s, 12H, $-\mathrm{OMe}$ ), 3.50 (s, $12 \mathrm{H},-\mathrm{OMe}), 3.44-3.30\left(\mathrm{br}, 40 \mathrm{H},-\mathrm{CH}_{2}-\right.$ ), $3.26(\mathrm{~s}, 12 \mathrm{H}$, -OMe), 3.23 (s, $\left.12 \mathrm{H}, \mathrm{CH}_{3}\right), 3.17$ (s, $\left.12 \mathrm{H}, \mathrm{CH}_{3}\right), 3.15$ (s, $24 \mathrm{H}, \mathrm{CH}_{3}$ ), $3.08\left(\mathrm{~s}, 12 \mathrm{H}, \mathrm{CH}_{3}\right), 3.06\left(\mathrm{~s}, 12 \mathrm{H}, \mathrm{CH}_{3}\right.$ ), $2.98\left(\mathrm{br}, 8 \mathrm{H},-\mathrm{CH}_{2}-\right), 2.95\left(\mathrm{~s}, 12 \mathrm{H}, \mathrm{CH}_{3}\right), 2.92\left(\mathrm{~s}, 24 \mathrm{H}, \mathrm{CH}_{3}\right), 2.90$ (s, 24H, $\left.\mathrm{CH}_{3}\right), 2.87\left(\mathrm{~s}, 12 \mathrm{H}, \mathrm{CH}_{3}\right), 2.85$ (s, $\left.24 \mathrm{H}, \mathrm{CH}_{3}\right), 2.60(\mathrm{~s}, 12 \mathrm{H},-\mathrm{OMe})$.

Physical data of 1•TMSA: ${ }^{1} \mathrm{H}$ NMR (acetonitrile- $\left.d_{3} / \mathrm{D}_{2} \mathrm{O}(1: 4 \mathrm{v} / \mathrm{v}), 500 \mathrm{MHz}\right): \delta 10.02(\mathrm{~d}, J=6.0 \mathrm{~Hz}, 4 \mathrm{H}$, $\operatorname{Ar} H$ ), 9.95 (d, $J=6.0 \mathrm{~Hz}, 4 \mathrm{H}, \operatorname{Ar} H$ ), 9.60 (d, $J=5.5 \mathrm{~Hz}, 8 \mathrm{H}, \operatorname{Ar} H), 9.53$ (d, $J=5.5 \mathrm{~Hz}, 8 \mathrm{H}, \operatorname{Ar} H), 9.44$ (d, $J=6.0 \mathrm{~Hz}, 4 \mathrm{H}, \operatorname{Ar} H), 9.31(\mathrm{~d}, J=5.5 \mathrm{~Hz}, 12 \mathrm{H}, \operatorname{Ar} H), 9.16(\mathrm{~d}, J=6.0 \mathrm{~Hz}, 4 \mathrm{H}, \operatorname{Ar} H), 9.13(\mathrm{~d}, J=6.0 \mathrm{~Hz}$, 4H, $\operatorname{Ar} H), 8.00(\mathrm{~d}, J=5.5 \mathrm{~Hz}, 4 \mathrm{H}, \operatorname{Ar} H), 7.92(\mathrm{~d}, J=5.5 \mathrm{~Hz}, 4 \mathrm{H}, \operatorname{Ar} H), 7.89$ (d, $J=5.0 \mathrm{~Hz}, 4 \mathrm{H}, \operatorname{Ar} H), 7.73$ (d, $J=6.5 \mathrm{~Hz}, 4 \mathrm{H}, \operatorname{Ar} H$ ), 7.68 (d, $J=6.5 \mathrm{~Hz}, 8 \mathrm{H}, \operatorname{Ar} H), 7.59$ (d, $J=4.5 \mathrm{~Hz}, 8 \mathrm{H}, \operatorname{Ar} H), 7.55$ (br, 4H, $\operatorname{Ar} H$ ), 7.52 (d, $J=6.0 \mathrm{~Hz}, 4 \mathrm{H}, \operatorname{Ar} H), 7.41(\mathrm{~d}, J=5.0 \mathrm{~Hz}, 4 \mathrm{H}, \operatorname{Ar} H), 7.23(\mathrm{~d}, J=3.5 \mathrm{~Hz}, 4 \mathrm{H}, \operatorname{Ar} H), 7.20$ (d, $J=$ $5.0 \mathrm{~Hz}, 4 \mathrm{H}, \operatorname{Ar} H), 7.15$ (d, $J=4.5 \mathrm{~Hz}, 8 \mathrm{H}, \operatorname{Ar} H), 6.85$ (d, $J=4.0 \mathrm{~Hz}, 4 \mathrm{H}, \operatorname{Ar} H), 6.81$ (d, $J=3.5 \mathrm{~Hz}, 4 \mathrm{H}$, $\operatorname{Ar} H), 6.76(\mathrm{~d}, J=4.0 \mathrm{~Hz}, 4 \mathrm{H}, \operatorname{Ar} H), 6.07$ (d, $J=3.5 \mathrm{~Hz}, 4 \mathrm{H}, \operatorname{Ar} H), 5.70$ (d, $J=3.0 \mathrm{~Hz}, 4 \mathrm{H}, \operatorname{Ar} H), 5.42(\mathrm{~d}$, $J=3.5 \mathrm{~Hz}, 4 \mathrm{H}, \operatorname{Ar} H$ ), 5.19 (br, 4H, $\operatorname{Ar} H$ ), 5.02 (d, $J=3.0 \mathrm{~Hz}, 4 \mathrm{H}, \operatorname{Ar} H), 4.93(\mathrm{~d}, J=3.5 \mathrm{~Hz}, 4 \mathrm{H}, \operatorname{Ar} H$ ), $4.73(\mathrm{~d}, J=3.5 \mathrm{~Hz}, 4 \mathrm{H}, \mathrm{Ar} H$ ), 4.05 (s, 12H, $-\mathrm{OMe}$ ), 3.69 (s, 12H, $-\mathrm{O} M e), 3.55$ (s, 12H, $-\mathrm{O} M e$ ), 3.49 (s, $12 \mathrm{H},-\mathrm{OMe}$ ), 3.44-3.31 (br, 40H, $\left.-\mathrm{CH}_{2}-\right), 3.28$ (s, $\left.12 \mathrm{H},-\mathrm{OMe}\right), 3.27$ (s, $\left.12 \mathrm{H}, \mathrm{CH}_{3}\right), 3.25\left(\mathrm{~s}, 12 \mathrm{H}, \mathrm{CH}_{3}\right)$, $3.17\left(\mathrm{~s}, 12 \mathrm{H}, \mathrm{CH}_{3}\right), 3.16\left(\mathrm{~s}, 24 \mathrm{H}, \mathrm{CH}_{3}\right), 3.10\left(\mathrm{~s}, 12 \mathrm{H}, \mathrm{CH}_{3}\right), 3.02\left(\mathrm{~s}, 12 \mathrm{H}, \mathrm{CH}_{3}\right), 2.99$ (br, $\left.8 \mathrm{H},-\mathrm{CH}_{2}-\right), 2.97$ (s, $\left.12 \mathrm{H}, \mathrm{CH}_{3}\right), 2.93$ (s, 12H, $\mathrm{CH}_{3}$ ) 2.91 (s, 24H, $\left.\mathrm{CH}_{3}\right), 2.89$ (s, 12H, CH $\left.\mathrm{CH}_{3}\right), 2.67$ (s, 12H, -OMe), -0.61 (s, $1 \mathrm{H}, \mathrm{CH}),-2.35\left(\mathrm{~s}, 9 \mathrm{H}, \mathrm{CH}_{3}\right)$. 
(a)

cage 1

(b) 1. $\mathrm{CHCl}_{3}$ (-)

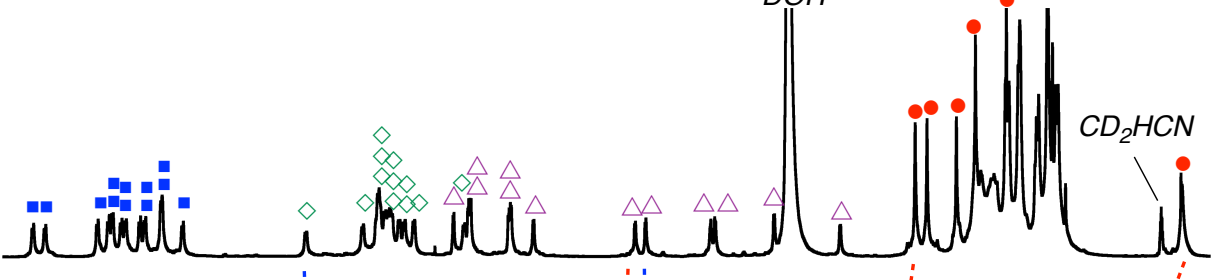

(c) $\mathbf{1} \cdot \mathrm{CH}_{2} \mathrm{I}_{2}$
$(49 \%)$

(d) 1. $\mathrm{CHBr}_{3}$ (95\%)

(e) $1 \cdot \mathrm{CCl}_{4}$ (92\%)

(f) $1 \cdot \mathrm{CHI}_{3}$ (5\%)

(g) 1. $\mathrm{CBr}_{4}$ (93\%)

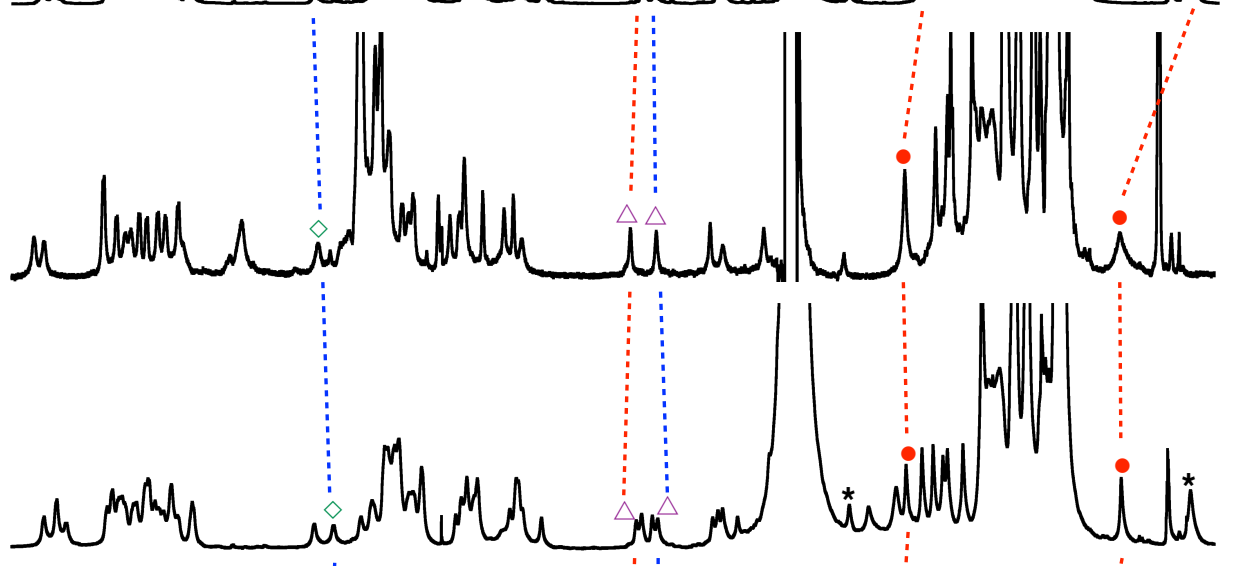


(a)

cage 1

(b) $1 \cdot\left(\mathrm{CHCl}_{2}\right)_{2}$ (85\%)

\section{$1 \cdot$ TMSA}

(c) $(63 \%)$

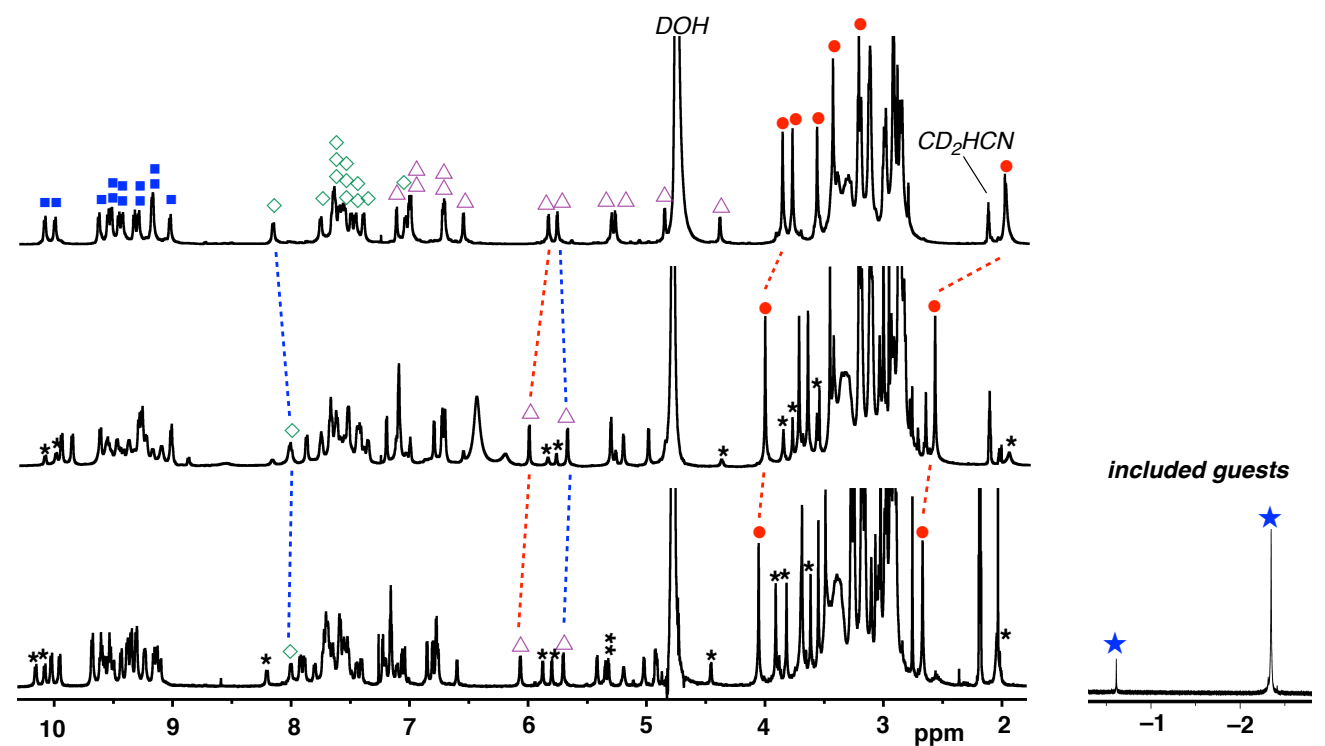

Figure S29. ${ }^{1} \mathrm{H}$ NMR spectra $\left(500 \mathrm{MHz}, 300 \mathrm{~K}\right.$, acetonitrile- $\left.d_{3} / \mathrm{D}_{2} \mathrm{O}(1: 4 \mathrm{v} / \mathrm{v})\right)$ of (a) $\mathbf{1}$, (b) $\mathbf{1} \bullet(\mathbf{C} \mathbf{H C l})_{2}$, and (c) 1•TMSA.

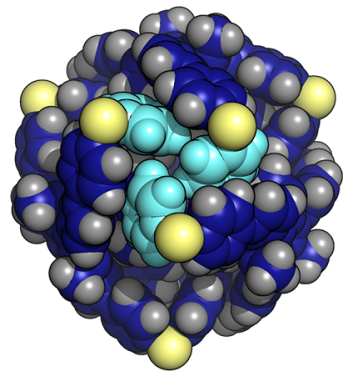

before inclusion

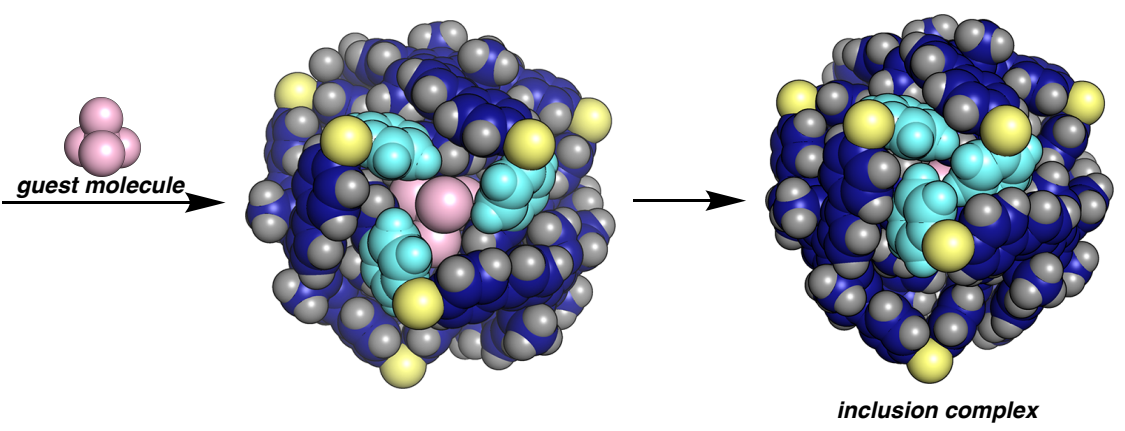

Figure S30. Proposed mechanism of inclusion 


\section{Inclusion experiments in $\mathrm{D}_{2} \mathrm{O}$}

\section{General procedure}

Dried powder of cage $1(18.0 \mathrm{mg}, 2.0 \mu \mathrm{mol})$ was dissolved in $\mathrm{D}_{2} \mathrm{O}(800 \mu \mathrm{L})$. Guest molecule (100 eq.) was added to the solution and stirred at room temperature for $10 \mathrm{~min}$. The formation of the inclusion complex was confirmed by ${ }^{1} \mathrm{H}$ NMR spectroscopy. The yields of the inclusion complex were determined by comparison of the integral ratio between the signals from empty cage $\mathbf{1}$ and the guest included cage $\mathbf{1} \cdot \mathbf{G}$.

Physical data of 1: ${ }^{1} \mathrm{H}$ NMR ( $\left.\mathrm{D}_{2} \mathrm{O}, 500 \mathrm{MHz}\right): \delta 9.92(\mathrm{~d}, J=6.0 \mathrm{~Hz}, 4 \mathrm{H}, \operatorname{Ar} H), 9.80(\mathrm{~d}, J=6.0 \mathrm{~Hz}, 4 \mathrm{H}$, $\operatorname{Ar} H$ ), 9.57 (d, $J=6.0 \mathrm{~Hz}, 4 \mathrm{H}, \operatorname{Ar} H), 9.46(\mathrm{~d}, J=6.0 \mathrm{~Hz}, 4 \mathrm{H}, \operatorname{Ar} H), 9.44$ (d, $J=6.5 \mathrm{~Hz}, 4 \mathrm{H}, \operatorname{Ar} H), 9.29$ (d, $J=6.0 \mathrm{~Hz}, 4 \mathrm{H}, \operatorname{Ar} H), 9.27(\mathrm{~d}, J=6.5 \mathrm{~Hz}, 4 \mathrm{H}, \operatorname{Ar} H), 9.26(\mathrm{~d}, J=6.0 \mathrm{~Hz}, 4 \mathrm{H}, \operatorname{Ar} H), 9.22(\mathrm{~d}, J=6.0 \mathrm{~Hz}$, 4H, $\operatorname{Ar} H), 9.10(\mathrm{~d}, J=6.0 \mathrm{~Hz}, 4 \mathrm{H}, \operatorname{Ar} H), 9.08(\mathrm{~d}, J=6.5 \mathrm{~Hz}, 4 \mathrm{H}, \operatorname{Ar} H), 8.87(\mathrm{~d}, J=6.0 \mathrm{~Hz}, 4 \mathrm{H}, \operatorname{Ar} H), 8.14$ $(\mathrm{d}, J=5.0 \mathrm{~Hz}, 4 \mathrm{H}, \operatorname{Ar} H), 7.70(\mathrm{~d}, J=5.0 \mathrm{~Hz}, 4 \mathrm{H}, \operatorname{Ar} H), 7.63(\mathrm{~d}, J=5.0 \mathrm{~Hz}, 4 \mathrm{H}, \operatorname{Ar} H), 7.59(\mathrm{~d}, J=5.0 \mathrm{~Hz}$, 4H, $\operatorname{Ar} H), 7.52(\mathrm{~d}, J=6.5 \mathrm{~Hz}, 8 \mathrm{H}, \operatorname{Ar} H), 7.51(\mathrm{~d}, J=5.0 \mathrm{~Hz}, 4 \mathrm{H}, \operatorname{Ar} H), 7.44(\mathrm{~d}, J=6.0 \mathrm{~Hz}, 4 \mathrm{H}, \operatorname{Ar} H), 7.42$ (d, $J=6.5 \mathrm{~Hz}, 4 \mathrm{H}, \operatorname{Ar} H), 7.33(\mathrm{~d}, J=5.0 \mathrm{~Hz}, 4 \mathrm{H}, \operatorname{Ar} H), 7.32(\mathrm{~d}, J=5.0 \mathrm{~Hz}, 4 \mathrm{H}, \operatorname{Ar} H), 7.07$ (d, $J=3.5 \mathrm{~Hz}$, 4H, $\operatorname{Ar} H), 6.94(\mathrm{~d}, J=4.0 \mathrm{~Hz}, 4 \mathrm{H}, \operatorname{Ar} H), 6.92(\mathrm{~d}, J=4.0 \mathrm{~Hz}, 8 \mathrm{H}, \operatorname{Ar} H), 6.66(\mathrm{~d}, J=3.5 \mathrm{~Hz}, 4 \mathrm{H}, \operatorname{Ar} H), 6.64$ (d, $J=3.5 \mathrm{~Hz}, 4 \mathrm{H}, \operatorname{Ar} H), 6.48(\mathrm{~d}, J=3.5 \mathrm{~Hz}, 4 \mathrm{H}, \operatorname{Ar} H), 5.81(\mathrm{~d}, J=3.5 \mathrm{~Hz}, 4 \mathrm{H}, \operatorname{Ar} H), 5.75(\mathrm{~d}, J=3.5 \mathrm{~Hz}$, 4H, $\operatorname{Ar} H$ ), 5.29 (d, $J=3.5 \mathrm{~Hz}, 4 \mathrm{H}, \operatorname{Ar} H), 5.20$ (d, $J=3.5 \mathrm{~Hz}, 4 \mathrm{H}, \operatorname{Ar} H), 4.76(\mathrm{~d}, J=3.5 \mathrm{~Hz}, 4 \mathrm{H}, \operatorname{Ar} H), 4.23$ (d, $J=3.5 \mathrm{~Hz}, 4 \mathrm{H}, \operatorname{Ar} H), 3.78$ (s, 12H, -OMe), 3.76 (s, 12H, -OMe), 3.51 (s, 12H, -OMe), 3.43-3.21 (br, $\left.40 \mathrm{H},-\mathrm{CH}_{2}-\right), 3.35$ (s, 12H, -OMe), $3.18\left(\mathrm{~s}, 12 \mathrm{H}, \mathrm{CH}_{3}\right.$ ), 3.14 (s, $\left.12 \mathrm{H},-\mathrm{OMe}\right), 3.13\left(\mathrm{~s}, 12 \mathrm{H}, \mathrm{CH}_{3}\right), 3.08$ (br, $\left.8 \mathrm{H},-\mathrm{CH}_{2}-\right), 3.05\left(\mathrm{~s}, 24 \mathrm{H}, \mathrm{CH}_{3}\right), 2.94\left(\mathrm{~s}, 12 \mathrm{H}, \mathrm{CH}_{3}\right), 2.93\left(\mathrm{~s}, 12 \mathrm{H}, \mathrm{CH}_{3}\right), 2.89\left(\mathrm{~s}, 24 \mathrm{H}, \mathrm{CH}_{3}\right), 2.87(\mathrm{~s}, 24 \mathrm{H}$, $\left.\mathrm{CH}_{3}\right), 2.82\left(\mathrm{~s}, 12 \mathrm{H}, \mathrm{CH}_{3}\right), 2.77\left(\mathrm{~s}, 12 \mathrm{H}, \mathrm{CH}_{3}\right), 1.76(\mathrm{~s}, 12 \mathrm{H},-\mathrm{OMe})$. 
Physical data of 1•CCl ${ }_{4}:{ }^{1} \mathrm{H}$ NMR $\left(\mathrm{D}_{2} \mathrm{O}, 500 \mathrm{MHz}\right): \delta 9.77(\mathrm{~d}, J=6.0 \mathrm{~Hz}, 4 \mathrm{H}, \mathrm{Ar} H), 9.63(\mathrm{~d}, J=5.5 \mathrm{~Hz}$, 4H, $\operatorname{Ar} H$ ), 9.51 (d, $J=6.0 \mathrm{~Hz}, 4 \mathrm{H}, \operatorname{Ar} H$ ), 9.49 (d, $J=5.5 \mathrm{~Hz}, 4 \mathrm{H}, \operatorname{Ar} H$ ), 9.41 (d, $J=5.5 \mathrm{~Hz}, 4 \mathrm{H}, \operatorname{Ar} H$ ), 9.30 (d, $J=6.0 \mathrm{~Hz}, 4 \mathrm{H}, \operatorname{Ar} H), 9.20$ (d, $J=6.0 \mathrm{~Hz}, 4 \mathrm{H}, \operatorname{Ar} H), 9.15$ (br, 12H, $\operatorname{Ar} H), 9.02(\mathrm{~d}, J=6.0 \mathrm{~Hz}, 4 \mathrm{H}, \operatorname{Ar} H$ ), $8.85(\mathrm{~d}, J=6.0 \mathrm{~Hz}, 4 \mathrm{H}, \operatorname{Ar} H), 7.98(\mathrm{~d}, J=4.5 \mathrm{~Hz}, 4 \mathrm{H}, \operatorname{Ar} H), 7.80(\mathrm{~d}, J=5.0 \mathrm{~Hz}, 4 \mathrm{H}, \operatorname{Ar} H), 7.66$ (d, $J=$ $5.0 \mathrm{~Hz}, 4 \mathrm{H}, \operatorname{Ar} H), 7.61(\mathrm{br}, 12 \mathrm{H}, \operatorname{Ar} H), 7.45(\mathrm{~d}, J=5.0 \mathrm{~Hz}, 8 \mathrm{H}, \operatorname{Ar} H), 7.33(\mathrm{~d}, J=5.0 \mathrm{~Hz}, 4 \mathrm{H}, \operatorname{Ar} H), 7.27$ $(\mathrm{d}, J=5.0 \mathrm{~Hz}, 4 \mathrm{H}, \operatorname{Ar} H), 7.26(\mathrm{~d}, J=5.0 \mathrm{~Hz}, 4 \mathrm{H}, \operatorname{Ar} H), 7.20$ (d, $J=3.0 \mathrm{~Hz}, 4 \mathrm{H}, \operatorname{Ar} H), 7.05(\mathrm{~d}, J=3.0 \mathrm{~Hz}$, 4H, $\operatorname{Ar} H), 6.99(\mathrm{~d}, J=3.0 \mathrm{~Hz}, 4 \mathrm{H}, \operatorname{Ar} H), 6.90(\mathrm{~d}, J=4.5 \mathrm{~Hz}, 4 \mathrm{H}, \operatorname{Ar} H), 6.76(\mathrm{~d}, J=3.0 \mathrm{~Hz}, 4 \mathrm{H}, \operatorname{Ar} H), 6.69$ $(\mathrm{d}, J=3.0 \mathrm{~Hz}, 4 \mathrm{H}, \operatorname{Ar} H), 6.58(\mathrm{~d}, J=3.0 \mathrm{~Hz}, 4 \mathrm{H}, \operatorname{Ar} H), 5.91(\mathrm{~d}, J=3.5 \mathrm{~Hz}, 4 \mathrm{H}, \operatorname{Ar} H), 5.60(\mathrm{~d}, J=2.5 \mathrm{~Hz}$, 4H, $\operatorname{Ar} H), 5.25$ (d, $J=3.0 \mathrm{~Hz}, 4 \mathrm{H}, \operatorname{Ar} H), 5.14(\mathrm{~d}, J=2.5 \mathrm{~Hz}, 4 \mathrm{H}, \operatorname{Ar} H), 4.91$ (d, $J=3.5 \mathrm{~Hz}, 4 \mathrm{H}, \operatorname{Ar} H), 4.63$ (d, J=3.0 Hz, 4H, $\operatorname{Ar} H$ ), 3.98 (s, 12H, -OMe), 3.68 (s, 12H, -OMe), $3.62(\mathrm{~s}, 12 \mathrm{H},-\mathrm{O} M e), 3.39$ (s, 12H, $\mathrm{OMe}$ ), 3.35-3.20 (br, 40H, - $\mathrm{CH}_{2}-$ ), 3.17 (s, $12 \mathrm{H}, \mathrm{CH}_{3}$ ), 3.16 (s, 12H, $-\mathrm{OMe}$ ), 3.09 (s, 12H, $\mathrm{CH}_{3}$ ), 3.07 (s, $\left.12 \mathrm{H}, \mathrm{CH}_{3}\right), 3.04\left(\mathrm{~s}, 12 \mathrm{H}, \mathrm{CH}_{3}\right), 3.00\left(\mathrm{~s}, 12 \mathrm{H}, \mathrm{CH}_{3}\right), 2.90\left(\mathrm{br}, 8 \mathrm{H},-\mathrm{CH}_{2}-\right), 2.84\left(\mathrm{~s}, 12 \mathrm{H}, \mathrm{CH}_{3}\right), 2.83(\mathrm{~s}, 12 \mathrm{H}$, $\left.\mathrm{CH}_{3}\right), 2.81\left(\mathrm{~s}, 36 \mathrm{H}, \mathrm{CH}_{3}\right), 2.78\left(\mathrm{~s}, 24 \mathrm{H}, \mathrm{CH}_{3}\right), 2.58(\mathrm{~s}, 12 \mathrm{H},-\mathrm{OMe})$.

Physical data of 1•CHBr 3 : ${ }^{1} \mathrm{H}$ NMR $\left(\mathrm{D}_{2} \mathrm{O}, 500 \mathrm{MHz}\right): \delta 9.82(\mathrm{~d}, J=6.0 \mathrm{~Hz}, 4 \mathrm{H}, \mathrm{Ar} H), 9.70(\mathrm{~d}, J=5.5$ Hz, 4H, ArH), 9.52 (d, $J=5.5 \mathrm{~Hz}, 8 \mathrm{H}, \operatorname{Ar} H), 9.43(\mathrm{~d}, J=6.0 \mathrm{~Hz}, 4 \mathrm{H}, \operatorname{Ar} H), 9.30(\mathrm{~d}, J=5.0 \mathrm{~Hz}, 4 \mathrm{H}, \operatorname{Ar} H)$, $9.20(\mathrm{~d}, J=6.0 \mathrm{~Hz}, 4 \mathrm{H}, \operatorname{Ar} H), 9.18(\mathrm{~d}, J=5.0 \mathrm{~Hz}, 8 \mathrm{H}, \operatorname{Ar} H), 9.14(\mathrm{~d}, J=5.5 \mathrm{~Hz}, 4 \mathrm{H}, \operatorname{Ar} H), 9.04(\mathrm{~d}, J=$ $6.0 \mathrm{~Hz}, 4 \mathrm{H}, \operatorname{Ar} H), 8.90$ (d, J=5.5 Hz, 4H, ArH), 7.99 (d, $J=5.0 \mathrm{~Hz}, 4 \mathrm{H}, \operatorname{Ar} H), 7.80$ (d, J=5.0 Hz, 4H, $\operatorname{Ar} H$ ), 7.65 (d, $J=6.0 \mathrm{~Hz}, 4 \mathrm{H}, \operatorname{Ar} H), 7.63$ (d, $J=5.5 \mathrm{~Hz}, 4 \mathrm{H}, \operatorname{Ar} H), 7.59$ (d, $J=5.5 \mathrm{~Hz}, 8 \mathrm{H}, \operatorname{Ar} H), 7.49$ (d, $J=5.5 \mathrm{~Hz}, 4 \mathrm{H}, \operatorname{Ar} H), 7.48(\mathrm{~d}, J=6.0 \mathrm{~Hz}, 4 \mathrm{H}, \operatorname{Ar} H), 7.35(\mathrm{~d}, J=5.0 \mathrm{~Hz}, 4 \mathrm{H}, \operatorname{Ar} H), 7.34(\mathrm{~d}, J=6.0 \mathrm{~Hz}$, 4H, ArH), $7.31(\mathrm{~d}, J=5.5 \mathrm{~Hz}, 4 \mathrm{H}, \operatorname{Ar} H), 7.15(\mathrm{~d}, J=3.5 \mathrm{~Hz}, 4 \mathrm{H}, \operatorname{Ar} H), 7.04(\mathrm{~d}, J=3.5 \mathrm{~Hz}, 4 \mathrm{H}, \operatorname{Ar} H), 6.99$ (d, $J=4.0 \mathrm{~Hz}, 4 \mathrm{H}, \operatorname{Ar} H$ ), 6.97 (d, $J=5.5 \mathrm{~Hz}, 4 \mathrm{H}, \operatorname{Ar} H), 6.74$ (d, $J=3.5 \mathrm{~Hz}, 4 \mathrm{H}, \operatorname{Ar} H), 6.66$ (d, $J=3.0 \mathrm{~Hz}$, 4H, ArH), $6.64(\mathrm{~d}, J=3.5 \mathrm{~Hz}, 4 \mathrm{H}, \operatorname{Ar} H), 5.88(\mathrm{~d}, J=3.0 \mathrm{~Hz}, 4 \mathrm{H}, \operatorname{Ar} H), 5.68(\mathrm{~d}, J=3.0 \mathrm{~Hz}, 4 \mathrm{H}, \operatorname{Ar} H), 5.30$ (d, $J=3.5 \mathrm{~Hz}, 4 \mathrm{H}, \operatorname{Ar} H), 5.13(\mathrm{~d}, J=3.0 \mathrm{~Hz}, 4 \mathrm{H}, \operatorname{Ar} H), 4.89$ (d, $J=3.0 \mathrm{~Hz}, 4 \mathrm{H}, \operatorname{Ar} H), 4.63$ (d, $J=3.5 \mathrm{~Hz}$, $4 \mathrm{H}, \mathrm{ArH}$ ), 3.94 (s, 12H, -OMe), 3.39 (s, 12H, $-\mathrm{OMe}$ ), 3.38-3.20 (br, 40H, $-\mathrm{CH}_{2}-$ ), 3.17 (s, 12H, $\mathrm{CH}_{3}$ ),

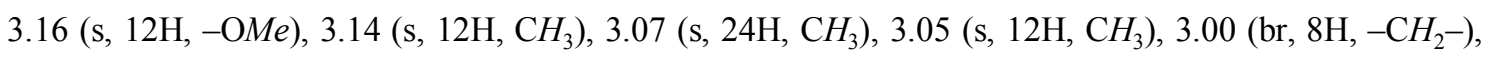
$2.91\left(\mathrm{~s}, 12 \mathrm{H}, \mathrm{CH}_{3}\right), 2.86\left(\mathrm{~s}, 12 \mathrm{H}, \mathrm{CH}_{3}\right), 2.84\left(\mathrm{~s}, 12 \mathrm{H}, \mathrm{CH}_{3}\right), 2.83\left(\mathrm{~s}, 24 \mathrm{H}, \mathrm{CH}_{3}\right), 2.82\left(\mathrm{~s}, 12 \mathrm{H}, \mathrm{CH}_{3}\right), 2.80(\mathrm{~s}$, $\left.12 \mathrm{H}, \mathrm{CH}_{3}\right), 2.52(\mathrm{~s}, 12 \mathrm{H},-\mathrm{OMe})$. 
Physical data of 1•CBr ${ }_{4}:{ }^{1} \mathrm{H}$ NMR $\left(\mathrm{D}_{2} \mathrm{O}, 500 \mathrm{MHz}\right): \delta 9.85(\mathrm{~d}, J=6.0 \mathrm{~Hz}, 4 \mathrm{H}, \mathrm{Ar} H), 9.72(\mathrm{~d}, J=6.0 \mathrm{~Hz}$, 4H, $\operatorname{Ar} H$ ), 9.59 (d, $J=6.0 \mathrm{~Hz}, 4 \mathrm{H}, \operatorname{Ar} H$ ), 9.51 (d, $J=5.5 \mathrm{~Hz}, 4 \mathrm{H}, \operatorname{Ar} H$ ), 9.43 (d, $J=6.0 \mathrm{~Hz}, 4 \mathrm{H}, \operatorname{Ar} H$ ), 9.35 $(\mathrm{d}, J=6.0 \mathrm{~Hz}, 4 \mathrm{H}, \operatorname{Ar} H), 9.24(\mathrm{~d}, J=6.5 \mathrm{~Hz}, 4 \mathrm{H}, \operatorname{Ar} H), 9.17(\mathrm{~d}, J=6.0 \mathrm{~Hz}, 4 \mathrm{H}, \operatorname{Ar} H), 9.16(\mathrm{~d}, J=6.0 \mathrm{~Hz}$, 8H, ArH), 9.04 (d, $J=6.5 \mathrm{~Hz}, 4 \mathrm{H}, \operatorname{Ar} H), 8.92(\mathrm{~d}, J=5.5 \mathrm{~Hz}, 4 \mathrm{H}, \operatorname{Ar} H), 8.00(\mathrm{~d}, J=5.0 \mathrm{~Hz}, 4 \mathrm{H}, \operatorname{Ar} H), 7.87$ (d, $J=4.5 \mathrm{~Hz}, 4 \mathrm{H}, \operatorname{Ar} H), 7.69(\mathrm{~d}, J=4.5 \mathrm{~Hz}, 8 \mathrm{H}, \operatorname{Ar} H), 7.65(\mathrm{~d}, J=6.0 \mathrm{~Hz}, 4 \mathrm{H}, \operatorname{Ar} H), 7.63(\mathrm{~d}, J=6.0 \mathrm{~Hz}$, 4H, $\operatorname{Ar} H$ ), $7.47(\mathrm{~d}, J=5.0 \mathrm{~Hz}, 8 \mathrm{H}, \operatorname{Ar} H$ ), $7.38(\mathrm{~d}, J=6.0 \mathrm{~Hz}, 4 \mathrm{H}, \operatorname{Ar} H$ ), 7.33 (d, $J=5.0 \mathrm{~Hz}, 4 \mathrm{H}, \operatorname{Ar} H$ ), 7.28 $(\mathrm{d}, J=5.5 \mathrm{~Hz}, 4 \mathrm{H}, \operatorname{Ar} H), 7.24(\mathrm{~d}, J=3.5 \mathrm{~Hz}, 4 \mathrm{H}, \operatorname{Ar} H), 7.10(\mathrm{~d}, J=4.0 \mathrm{~Hz}, 4 \mathrm{H}, \operatorname{Ar} H), 7.08$ (d, J=4.0 Hz, 4H, $\operatorname{Ar} H), 6.97(\mathrm{~d}, J=4.0 \mathrm{~Hz}, 4 \mathrm{H}, \operatorname{Ar} H), 6.81(\mathrm{~d}, J=3.5 \mathrm{~Hz}, 4 \mathrm{H}, \operatorname{Ar} H), 6.75(\mathrm{~d}, J=3.5 \mathrm{~Hz}, 4 \mathrm{H}, \operatorname{Ar} H), 6.64$ $(\mathrm{d}, J=3.5 \mathrm{~Hz}, 4 \mathrm{H}, \operatorname{Ar} H), 5.97(\mathrm{~d}, J=3.5 \mathrm{~Hz}, 4 \mathrm{H}, \operatorname{Ar} H), 5.63(\mathrm{~d}, J=3.0 \mathrm{~Hz}, 4 \mathrm{H}, \operatorname{Ar} H), 5.22(\mathrm{~d}, J=3.5 \mathrm{~Hz}$, 4H, $\operatorname{Ar} H$ ), $5.20(\mathrm{~d}, J=3.5 \mathrm{~Hz}, 4 \mathrm{H}, \operatorname{Ar} H), 4.96(\mathrm{~d}, J=3.5 \mathrm{~Hz}, 4 \mathrm{H}, \operatorname{Ar} H), 4.71(\mathrm{~d}, J=4.0 \mathrm{~Hz}, 4 \mathrm{H}, \operatorname{Ar} H), 4.06$ (s, 12H, -OMe), 3.76 (s, 12H, -OMe), 3.56 (s, 12H, -OMe), 3.44 (s, 12H, -OMe), 3.42-3.23 (br, 40H, $\left.\mathrm{CH}_{2}-\right), 3.20\left(\mathrm{~s}, 12 \mathrm{H}, \mathrm{CH}_{3}\right), 3.19(\mathrm{~s}, 12 \mathrm{H},-\mathrm{OMe}), 3.18\left(\mathrm{~s}, 12 \mathrm{H}, \mathrm{CH}_{3}\right), 3.12\left(\mathrm{~s}, 24 \mathrm{H}, \mathrm{CH}_{3}\right), 3.08(\mathrm{~s}, 12 \mathrm{H}$, $\left.\mathrm{CH}_{3}\right), 3.04\left(\mathrm{~s}, 12 \mathrm{H}, \mathrm{CH}_{3}\right), 2.97\left(\mathrm{br}, 8 \mathrm{H},-\mathrm{CH}_{2}-\right), 2.93\left(\mathrm{~s}, 24 \mathrm{H}, \mathrm{CH}_{3}\right), 2.86\left(\mathrm{~s}, 48 \mathrm{H}, \mathrm{CH}_{3}\right), 2.80(\mathrm{~s}, 12 \mathrm{H},-$ $\mathrm{OMe})$.

Physical data of $1 \cdot \mathbf{C H}_{2} \mathbf{I}_{2}:{ }^{1} \mathrm{H}$ NMR ( $\left.\mathrm{D}_{2} \mathrm{O}, 500 \mathrm{MHz}\right): \delta 9.80(\mathrm{~d}, J=5.5 \mathrm{~Hz}, 4 \mathrm{H}, \operatorname{Ar} H), 9.71(\mathrm{~d}, J=6.0 \mathrm{~Hz}$, 4H, $\operatorname{Ar} H$ ), 9.52 (d, $J=5.5 \mathrm{~Hz}, 4 \mathrm{H}, \operatorname{Ar} H), 9.50$ (d, $J=6.5 \mathrm{~Hz}, 4 \mathrm{H}, \operatorname{Ar} H), 9.43$ (d, $J=6.5 \mathrm{~Hz}, 4 \mathrm{H}, \operatorname{Ar} H), 9.30$ (d, $J=5.5 \mathrm{~Hz}, 4 \mathrm{H}, \operatorname{Ar} H), 9.18$ (d, $J=5.5 \mathrm{~Hz}, 12 \mathrm{H}, \operatorname{Ar} H), 9.14(\mathrm{~d}, J=5.5 \mathrm{~Hz}, 4 \mathrm{H}, \operatorname{Ar} H), 9.03(\mathrm{~d}, J=6.0$ $\mathrm{Hz}, 4 \mathrm{H}, \operatorname{Ar} H), 8.90(\mathrm{~d}, J=6.0 \mathrm{~Hz}, 4 \mathrm{H}, \operatorname{Ar} H), 7.98(\mathrm{~d}, J=5.5 \mathrm{~Hz}, 4 \mathrm{H}, \operatorname{Ar} H), 7.79(\mathrm{~d}, J=5.0 \mathrm{~Hz}, 4 \mathrm{H}, \operatorname{Ar} H)$, $7.63(\mathrm{~d}, J=5.5 \mathrm{~Hz}, 8 \mathrm{H}, \operatorname{Ar} H), 7.56(\mathrm{~d}, J=6.5 \mathrm{~Hz}, 8 \mathrm{H}, \operatorname{Ar} H), 7.50(\mathrm{~d}, J=7.0 \mathrm{~Hz}, 8 \mathrm{H}, \operatorname{Ar} H), 7.48(\mathrm{~d}, J=$ $7.0 \mathrm{~Hz}, 4 \mathrm{H}, \operatorname{Ar} H), 7.36(\mathrm{~d}, J=6.0 \mathrm{~Hz}, 4 \mathrm{H}, \operatorname{Ar} H), 7.31(\mathrm{~d}, J=6.5 \mathrm{~Hz}, 4 \mathrm{H}, \operatorname{Ar} H), 7.13(\mathrm{~d}, J=3.5 \mathrm{~Hz}, 4 \mathrm{H}$, $\operatorname{Ar} H), 7.03(\mathrm{~d}, J=3.5 \mathrm{~Hz}, 4 \mathrm{H}, \operatorname{Ar} H), 7.00(\mathrm{~d}, J=3.0 \mathrm{~Hz}, 8 \mathrm{H}, \operatorname{Ar} H), 6.72$ (d, $J=3.0 \mathrm{~Hz}, 4 \mathrm{H}, \operatorname{Ar} H), 6.66(\mathrm{~d}$, $J=3.0 \mathrm{~Hz}, 4 \mathrm{H}, \operatorname{Ar} H), 6.64(\mathrm{~d}, J=3.0 \mathrm{~Hz}, 4 \mathrm{H}, \operatorname{Ar} H), 5.84(\mathrm{~d}, J=3.0 \mathrm{~Hz}, 4 \mathrm{H}, \operatorname{Ar} H), 5.70(\mathrm{~d}, J=3.0 \mathrm{~Hz}$, 4H, $\operatorname{Ar} H), 5.29$ (br, 4H, ArH), 5.12 (d, $J=3.0 \mathrm{~Hz}, 4 \mathrm{H}, \operatorname{Ar} H), 4.88$ (d, $J=3.0 \mathrm{~Hz}, 4 \mathrm{H}, \operatorname{Ar} H), 4.66(\mathrm{~d}, J=$ $3.0 \mathrm{~Hz}, 4 \mathrm{H}, \mathrm{Ar} H$ ), 3.92 (s, 12H, -OMe), 3.67 (s, 12H, -OMe), 3.64 (s, 12H, -OMe), 3.47 (s, 12H, -OMe), 3.33-3.21 (br, $\left.40 \mathrm{H},-\mathrm{CH}_{2}-\right), 3.16(\mathrm{~s}, 12 \mathrm{H},-\mathrm{OMe}), 3.16\left(\mathrm{~s}, 12 \mathrm{H}, \mathrm{CH}_{3}\right), 3.13\left(\mathrm{~s}, 12 \mathrm{H}, \mathrm{CH}_{3}\right), 3.06(\mathrm{~s}, 24 \mathrm{H}$, $\mathrm{CH}_{3}$ ), 3.04 (s, $24 \mathrm{H}, \mathrm{CH}_{3}$ ), 2.97 (br, 8H, $\left.-\mathrm{CH}_{2}-\right), 2.90$ (s, $12 \mathrm{H}, \mathrm{CH}_{3}$ ), $2.85\left(\mathrm{~s}, 12 \mathrm{H}, \mathrm{CH}_{3}\right), 2.83\left(\mathrm{~s}, 12 \mathrm{H}, \mathrm{CH}_{3}\right)$, $2.81\left(\mathrm{~s}, 12 \mathrm{H}, \mathrm{CH}_{3}\right), 2.79\left(\mathrm{~s}, 24 \mathrm{H}, \mathrm{CH}_{3}\right), 2.41(\mathrm{~s}, 12 \mathrm{H},-\mathrm{OMe})$. 
(a)
cage 1

(b)

1. $\mathrm{CH}_{2} \mathrm{I}_{2}$

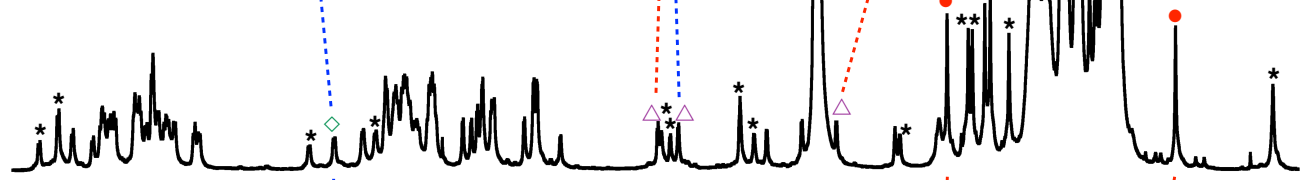

(c)

$\mathbf{1} \cdot \mathrm{CHBr}_{3}$
$(97 \%)$

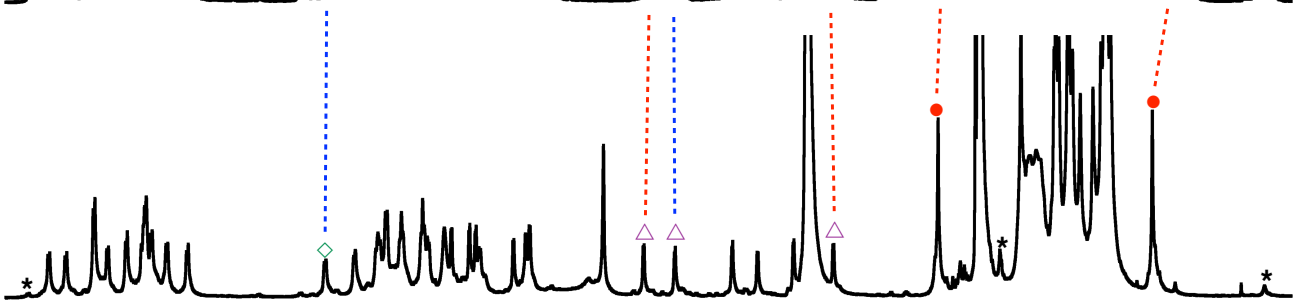

(d)
$\mathbf{1} \cdot \mathrm{CCl}_{4}$
$(96 \%)$

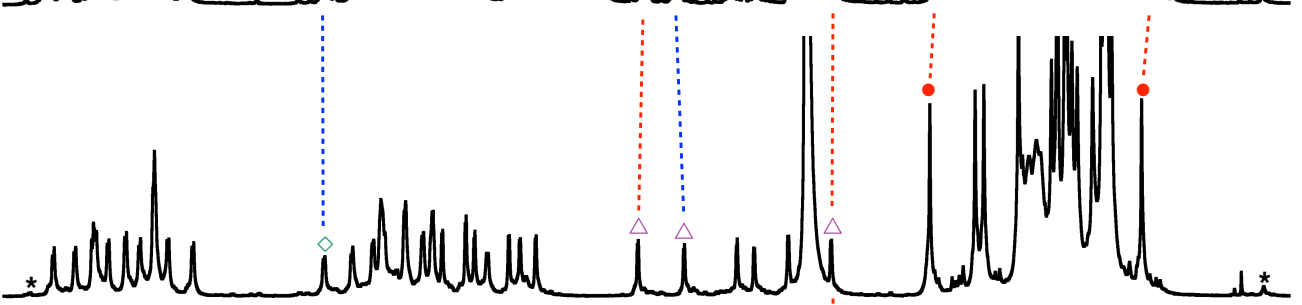

(e) $\mathrm{CHI}_{3}$

(11\%)
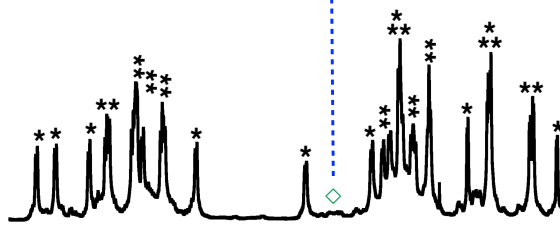

(f) $1 \cdot \mathrm{CBr}_{4}$

$(85 \%, 15 \mathrm{~h})$

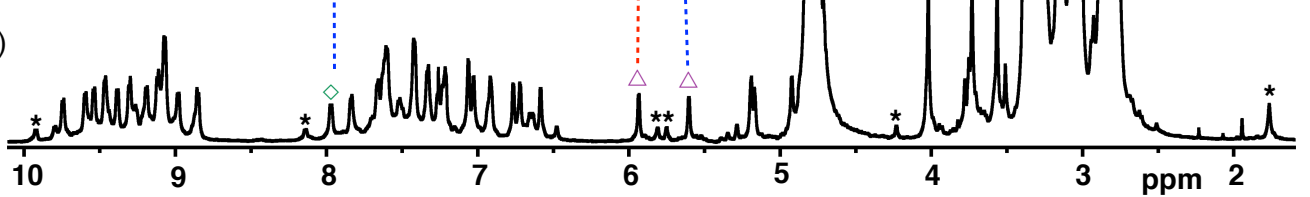

Figure S31. ${ }^{1} \mathrm{H}$ NMR spectra $\left(500 \mathrm{MHz}, 300 \mathrm{~K}, \mathrm{D}_{2} \mathrm{O}\right.$ ) of (a) $\mathbf{1}$, (b) $\mathbf{1} \cdot \mathbf{C H}_{\mathbf{2}} \mathbf{I}_{\mathbf{2}}$, (c) $\mathbf{1} \cdot \mathbf{C H B r}_{3}$, (d) $\mathbf{1} \cdot \mathbf{C C l}_{\mathbf{4}}$, (e) $1 \cdot \mathrm{CHI}_{3}$, and (f) $1 \cdot \mathrm{CBr}_{4}$. 


\section{X-ray crystallographic analyses of cage $1,1 \cdot \mathrm{CCl}_{4}$, and $\mathrm{S3}$}

\section{Procedures for crystallization}

\section{Cage 1}

An $\mathrm{CH}_{3} \mathrm{CN} / \mathrm{H}_{2} \mathrm{O}(1: 4 \mathrm{v} / \mathrm{v})$ solution of cage $\mathbf{1}$ was stood at $50^{\circ} \mathrm{C}$ and solvents were allowed to evaporate. After 10 days, a single crystal suitable for X-ray diffraction analysis was obtained.

\section{Inclusion complex $1 \cdot \mathrm{CCl}_{4}$}

Sat. $\mathrm{NaBF}_{4}$ aq. was added the $\mathrm{CH}_{3} \mathrm{CN} / \mathrm{H}_{2} \mathrm{O}(1: 4 \mathrm{v} / \mathrm{v})$ solution of cage $\mathbf{1}$. The obtained precipitate was washed with water and dried under reduced pressure. Cage 1 ' in which the counter anions were exchanged from $\mathrm{NO}_{3}^{-}$to $\mathrm{BF}_{4}^{-}$was dissolved in $\mathrm{CH}_{3} \mathrm{CN}$, an excess amount of $\mathrm{CCl}_{4}$ was added, and the mixture was stirred at room temperature for $10 \mathrm{~min}$. An excess amount of $\mathrm{NH}_{4} \mathrm{BF}_{4}$ was then added to the solution. The solution was stood at room temperature and the solvent were allowed to evaporate. After 1 day, a single crystal suitable for X-ray diffraction analysis was obtained. 
Table S1 Crystal data and structural refinement for $\mathbf{1}, \mathbf{1} \cdot \mathbf{C C l}_{4}$, and $\mathbf{S 3}$

\begin{tabular}{|c|c|c|c|}
\hline & 1 & $1 \cdot \mathrm{CCl}_{4}$ & S3 \\
\hline Identification code & $\mathrm{i}-42 \mathrm{~d}$ & ht1419d & $\mathrm{p}-1$ \\
\hline CCDC number & 1971564 & 1971565 & 1984215 \\
\hline Empirical formula & $\mathrm{C}_{713.58} \mathrm{H}_{804.32} \mathrm{~N}_{114.89} \mathrm{O}_{350.35} \mathrm{Pd}_{24}$ & $\mathrm{C}_{724} \mathrm{H}_{819} \mathrm{~B}_{48} \mathrm{Cl}_{8} \mathrm{~F}_{192} \mathrm{~N}_{97} \mathrm{O}_{110} \mathrm{Pd}_{24}$ & $\mathrm{C}_{21} \mathrm{H}_{18} \mathrm{O}_{6}$ \\
\hline Formula weight & 19148.69 & 19643.62 & 366.35 \\
\hline Temperature & $90(2) \mathrm{K}$ & $100.01(10) \mathrm{K}$ & $90(2) \mathrm{K}$ \\
\hline Wavelength & $0.71073 \AA$ & $1.54178 \AA$ & $0.71073 \AA$ \\
\hline Crystal system & Tetragonal & Monoclinic & Triclinic \\
\hline Space group & $I-42 \mathrm{~d}$ & $P 2_{1} / n$ & $P-1$ \\
\hline \multirow[t]{3}{*}{ Unit cell dimensions } & $a=b=40.908(11) \AA$ & $\begin{array}{l}a=35.2184(4) \AA \\
b=33.4270(4) \AA\end{array}$ & $\begin{array}{l}a=10.020(2) \AA \\
b=11.554(2) \AA\end{array}$ \\
\hline & $c=28.479(8) \AA$ & $c=37.3137(4) \AA$ & $c=16.035(3) \AA$ \\
\hline & $\alpha=\beta=\gamma=90^{\circ}$ & $\begin{array}{l}\alpha=\gamma=90^{\circ} \\
\beta=95.8588(10)^{\circ}\end{array}$ & $\begin{array}{l}\alpha=74.125(3)^{\circ} \\
\beta=84.067(3)^{\circ} \\
\gamma=85.833(3)^{\circ}\end{array}$ \\
\hline Volume & $47657(28) \AA^{3}$ & $43698.0(9) \AA^{3}$ & $1774.2(6) \AA^{3}$ \\
\hline$Z$ & 2 & 2 & 4 \\
\hline Density (calculated) & $1.334 \mathrm{Mg} / \mathrm{m}^{3}$ & $1.493 \mathrm{Mg} / \mathrm{m}^{3}$ & $1.372 \mathrm{Mg} / \mathrm{m}^{3}$ \\
\hline Absorption coefficient & $0.533 \mathrm{~mm}^{-1}$ & $5.072 \mathrm{~mm}^{-1}$ & $0.101 \mathrm{~mm}^{-1}$ \\
\hline$F(000)$ & 19547 & 19860 & 768 \\
\hline Crystal size & $0.14 \times 0.12 \times 0.09 \mathrm{~mm}^{3}$ & $0.07 \times 0.04 \times 0.03 \mathrm{~mm}^{3}$ & $0.28 \times 0.11 \times 0.04 \mathrm{~mm}^{3}$ \\
\hline Theta range for data & 0.996 to $26.460^{\circ}$ & 2.249 to $62.384^{\circ}$ & 1.325 to $28.313^{\circ}$ \\
\hline Index ranges & $\begin{array}{l}-51 \leqq h \leqq 51, \\
-51 \leqq k \leqq 51 \\
-35 \leqq l \leqq 35\end{array}$ & $\begin{array}{l}-40 \leqq h \leqq 39, \\
-36 \leqq k \leqq 38 \\
-42 \leqq l \leqq 38\end{array}$ & $\begin{array}{l}-13 \leqq h \leqq 13, \\
-15 \leqq k \leqq 15, \\
-20 \leqq l \leqq 15\end{array}$ \\
\hline Reflection collected & 247406 & 248790 & 14382 \\
\hline Independent reflections & $24590\left[R_{\mathrm{int}}=0.1389\right]$ & $68906\left[R_{\mathrm{int}}=0.1391\right]$ & $8192\left[R_{\mathrm{int}}=0.0314\right]$ \\
\hline Completeness & $99.9 \%\left(\theta=25.242^{\circ}\right)$ & $99.2 \%\left(\theta=62.384^{\circ}\right)$ & $98.9 \%\left(\theta=25.242^{\circ}\right)$ \\
\hline Max. and min. transmission & 0.7454 and 0.4738 & 1.00000 and 0.33030 & 0.7457 and 0.5879 \\
\hline Refinement method & & Full-matrix least-squares on $F^{2}$ & \\
\hline Data / restraints / parameters & $4590 / 1034 / 1803$ & $68906 / 2715 / 6128$ & $8192 / 0 / 493$ \\
\hline Goodness-of-fit on $F^{2}$ & 1.013 & 0.979 & 1.053 \\
\hline $\begin{array}{l}\text { Final } R \text { indices } \\
{[I>2 \sigma(I)]}\end{array}$ & $\begin{array}{l}R_{1}=0.0858 \\
w R_{2}=0.2218\end{array}$ & $\begin{array}{l}R_{1}=0.0905 \\
w R_{2}=0.2412\end{array}$ & $\begin{array}{l}R_{1}=0.0678 \\
w R_{2}=0.1853\end{array}$ \\
\hline$R$ indices (all data) & $\begin{array}{l}R_{1}=0.1422 \\
w R_{2}=0.2719\end{array}$ & $\begin{array}{l}R_{1}=0.2005 \\
w R_{2}=0.3146\end{array}$ & $\begin{array}{l}R_{1}=0.1000 \\
w R_{2}=0.2057\end{array}$ \\
\hline Absolute structure parameter & $0.144(10)$ & - & - \\
\hline Largest diff. peak and hole & 0.967 and $-0.513 \mathrm{e} \cdot \AA^{-3}$ & 1.754 and $-0.984 \mathrm{e} \cdot \AA^{-3}$ & 0.600 and -0.309 e. $\AA^{-3}$ \\
\hline
\end{tabular}




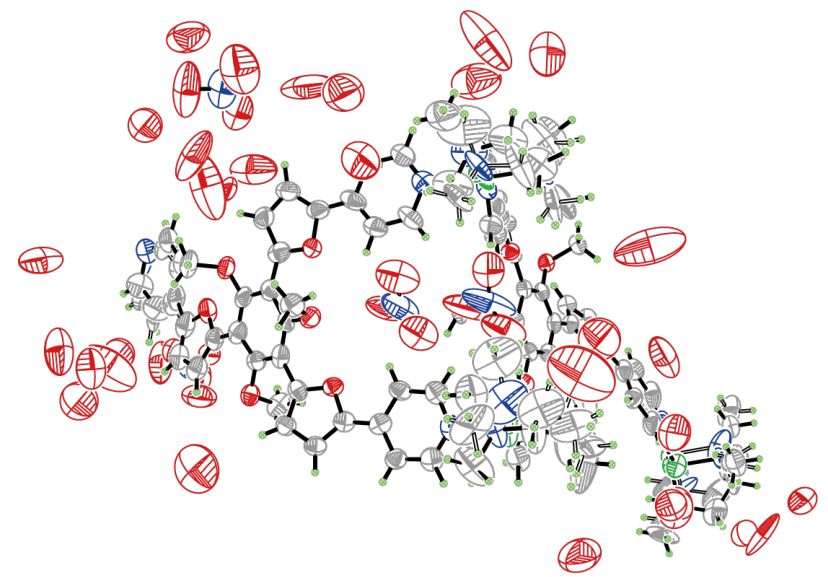

Figure S32. ORTEP drawings (50\% probability ellipsoids) of the refinement structure of 1.

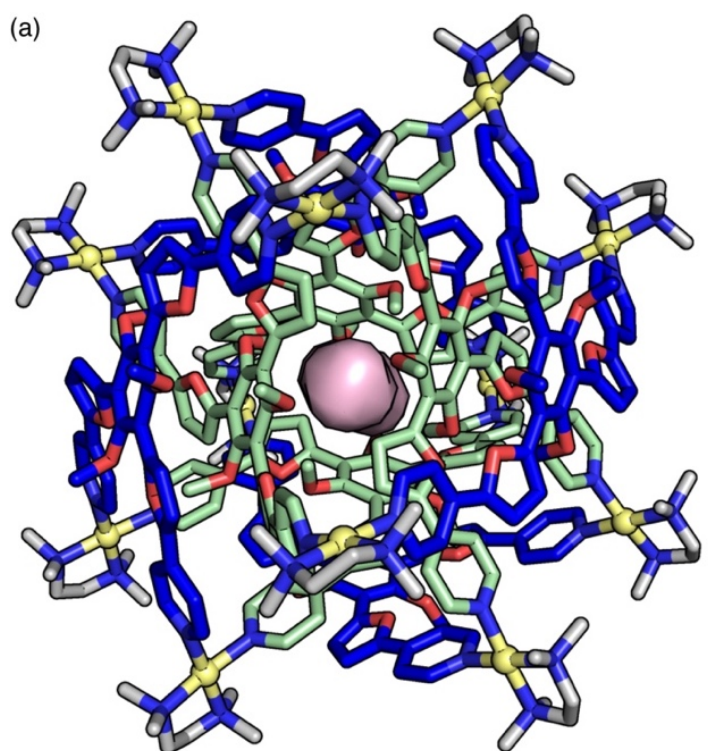

(b)
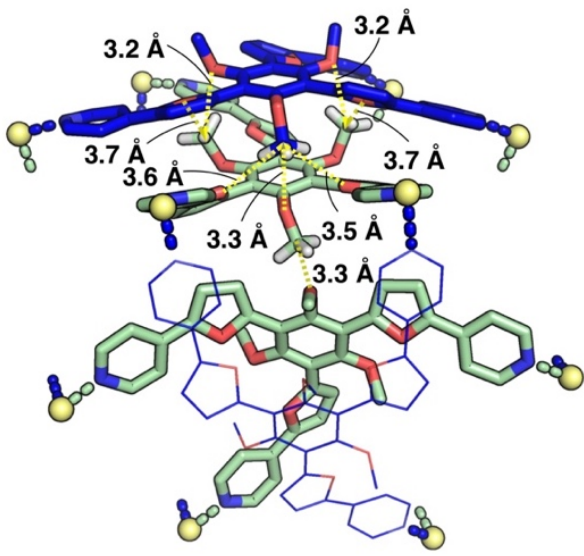

Figure S33. (a) Crystal structure of cage 1 (the pink object represents the cavity). (b) Hydrogen bonds between ligands of cage 1. Hydrogen atoms, solvent molecules and anions were omitted for clarity.
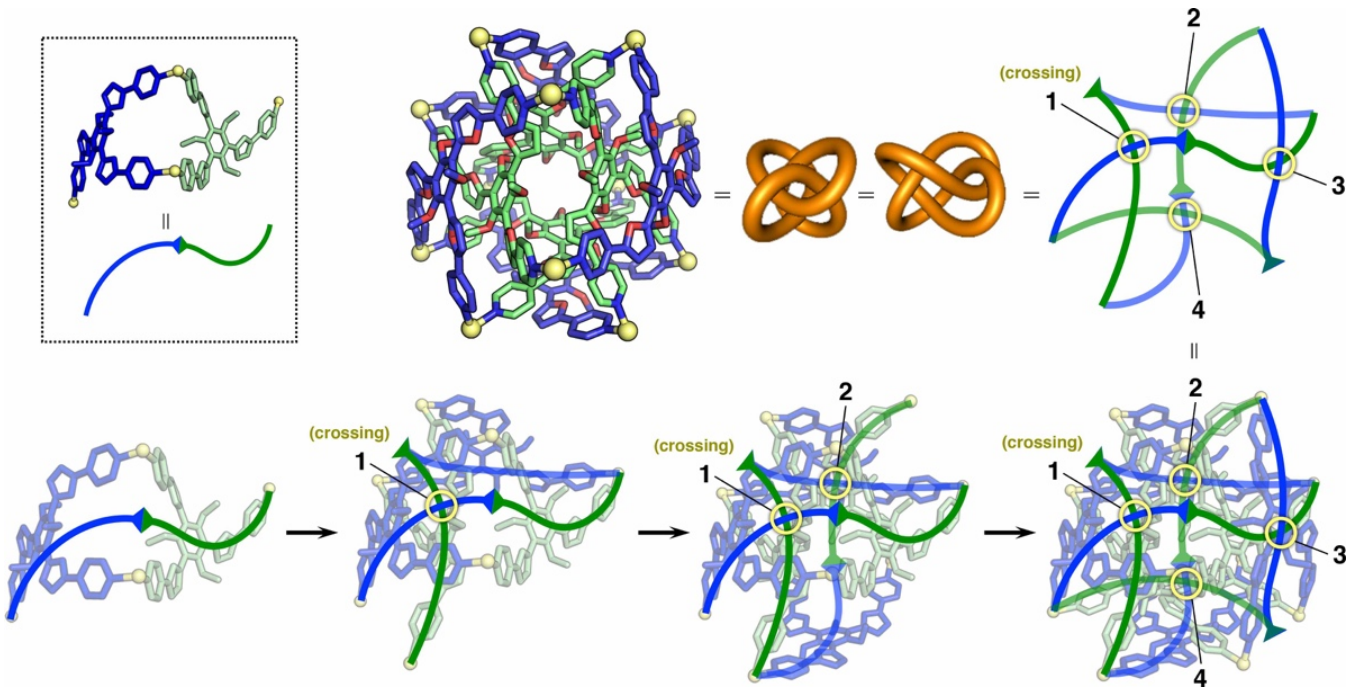

Figure S34. Schematic representation of the figure-eight $\left(4_{1}\right)$ knot topology of cage $1 .{ }^{\mathrm{S} 3}$ 

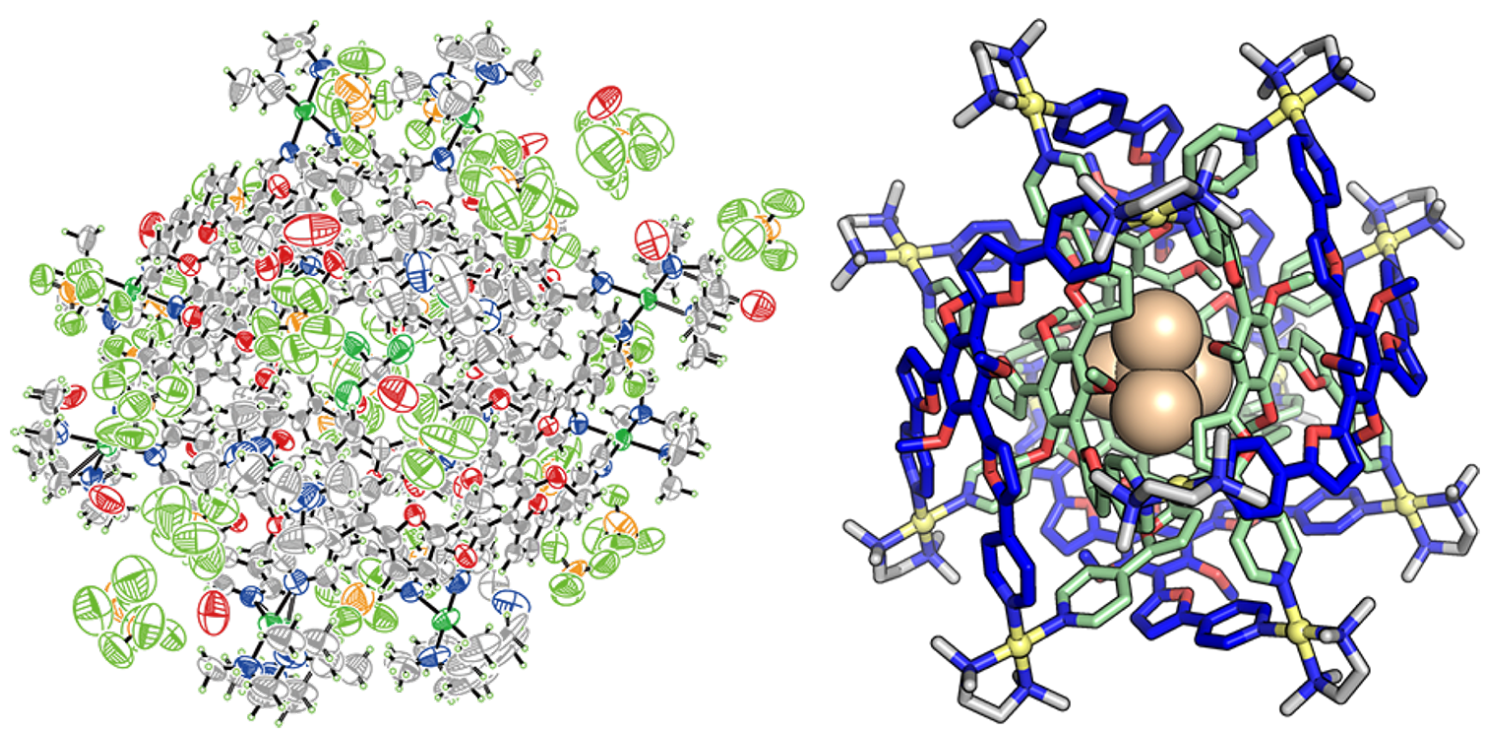

Figure S35. Crystal structure of $\mathbf{1} \cdot \mathbf{C C l}_{4}$. ORTEP drawings (50\% probability ellipsoids) of the refinement structure of $\mathbf{1} \cdot \mathbf{C C l}_{4}$ (left) and a stick model of $\mathbf{1} \cdot \mathbf{C C l}_{4}$ (right, the orange object represents $\mathrm{CCl}_{4}$ in the cavity. Hydrogen atoms, solvent molecules and anions were omitted for clarity).
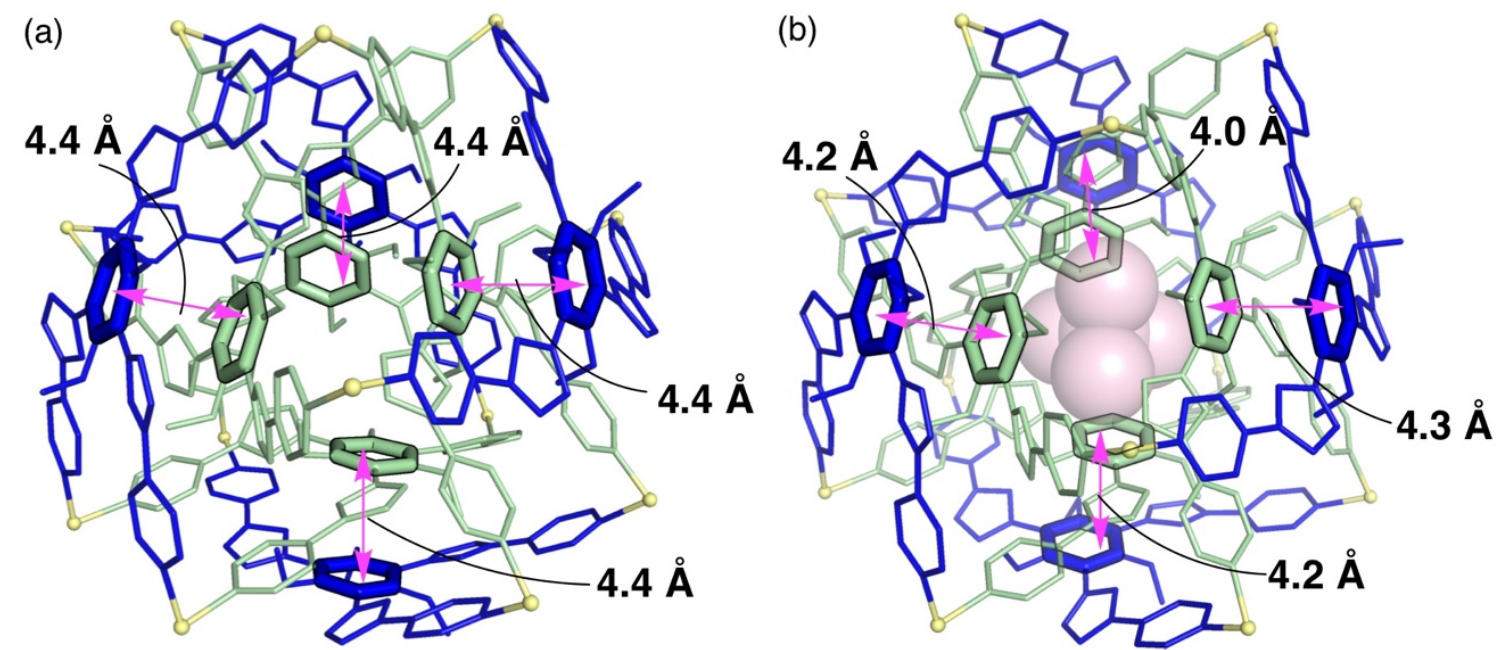

Figure S36. Distances between the benzene rings of outer and inner ligands in (a) cage $\mathbf{1}$ and (b) $\mathbf{1} \cdot \mathbf{C C l}_{\mathbf{4}}$. The distances were estimated with the averages of distances between six carbon atoms on a benzene ring of an inner ligand and the least-squares plane with six carbon atoms on the benzene ring of the correspondent outer ligand. 


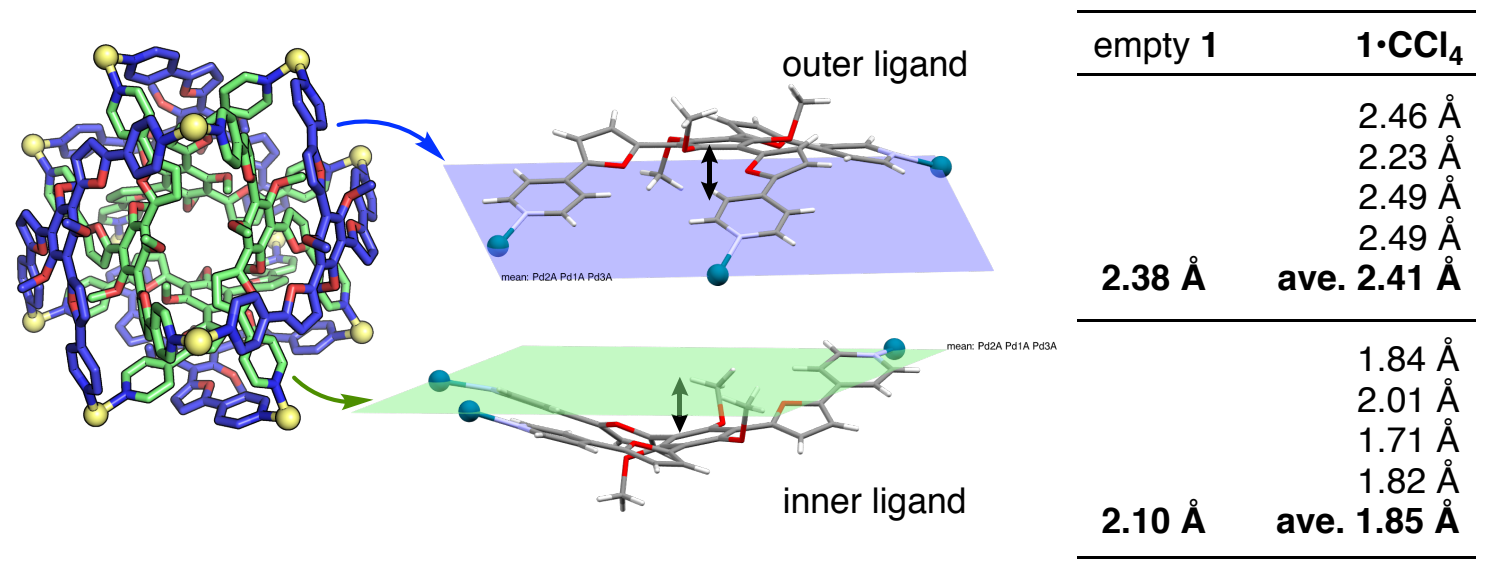

Figure S37. The averaged distances between the carbon atoms on the benzene core of the ligand and the plane passing through three Pd atoms connected to the ligand.

\section{Sequential guest binding}
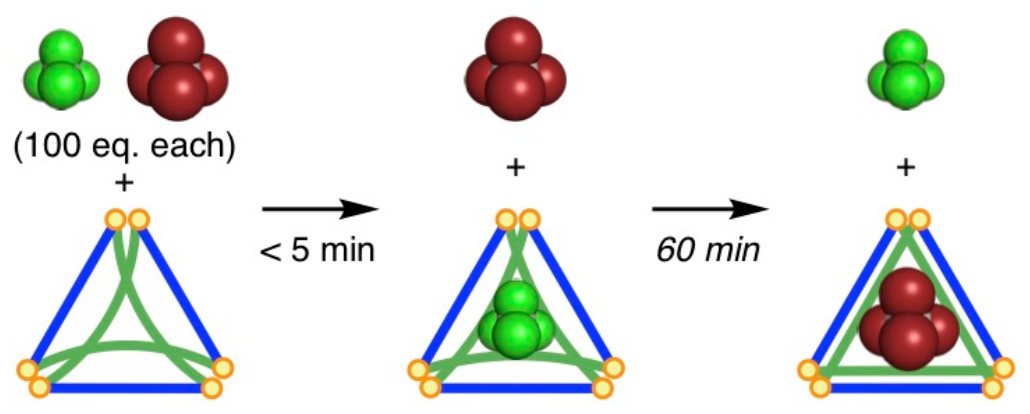

Dried powder of cage $1(18.0 \mathrm{mg}, 2.0 \mu \mathrm{mol})$ was dissolved in acetonitrile- $d_{3} / \mathrm{D}_{2} \mathrm{O}(800 \mu \mathrm{L}, 1: 4 \mathrm{v} / \mathrm{v})$. Guest molecules $\left(\mathrm{CCl}_{4}\right.$ and $\mathrm{CBr}_{4}, 100$ eq. each to cage 1) were added to the solution and ${ }^{1} \mathrm{H}$ NMR was measured immediately. ${ }^{1} \mathrm{H}$ NMR measurements were repeated after every 5 -minute stirring until the total stirring time reached $60 \mathrm{~min}$. The yields of the inclusion complex were determined by comparison of the integral ratios among the signals from empty cage $\mathbf{1}, \mathbf{1} \cdot \mathbf{C C l}_{4}$ and $\mathbf{1} \cdot \mathbf{C B r}_{4}$. 


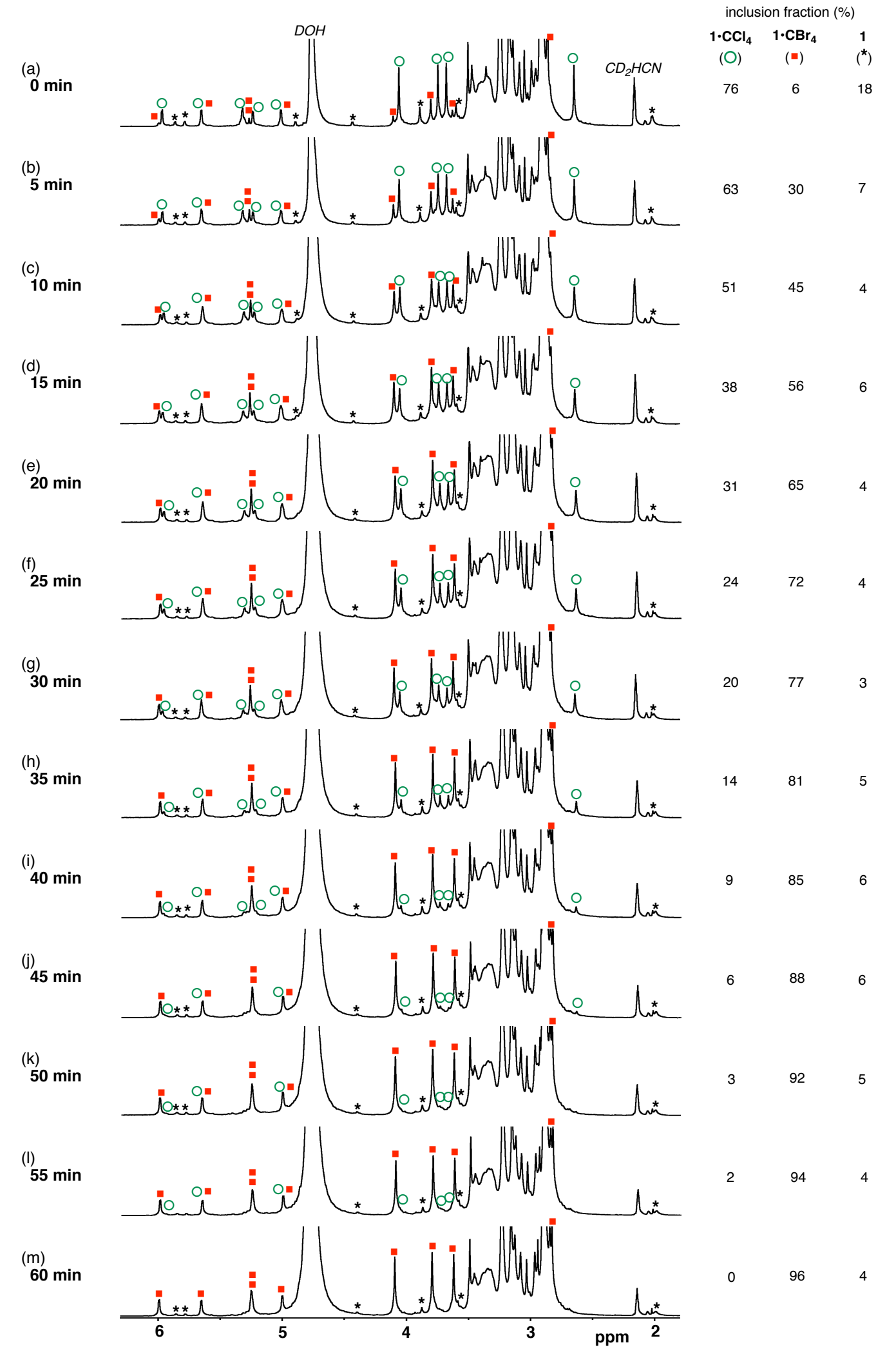

Figure S38. ${ }^{1} \mathrm{H}$ NMR spectra $\left(500 \mathrm{MHz}, 300 \mathrm{~K}\right.$, acetonitrile- $\left.d_{3} / \mathrm{D}_{2} \mathrm{O}(1: 4 \mathrm{v} / \mathrm{v})\right)$ during the sequential binding. 


\section{References}

[S1] E. Kiehlmannand, and R. W. Lauen, Can. J. Chem. 1989, 67, 335-344.

[S2] K. R. Idzik, R. Beckert, E. Tauscher, Pr. Ledwon, S. Golba, M. Lapkowski, P. Rapta, L. Dunsch, and J. Frydel, Mater. Sci. Forum., 2010, 663-665, 876-879.

[S3] The 3D models of the figure-eight knot topology were drawn by KnotPlot Version 1.0 (https://knotplot.com/). 